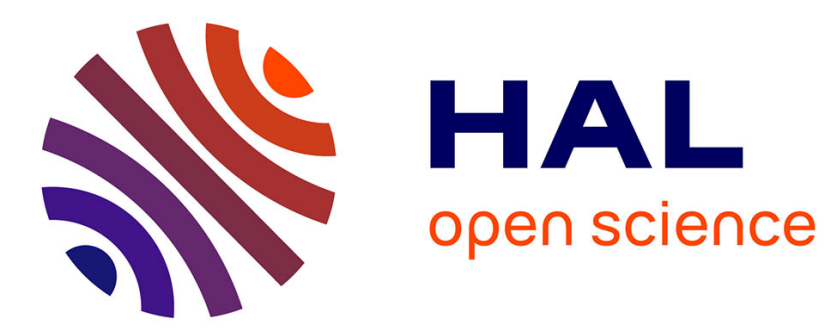

\title{
From Poly(Alkyl Cyanoacrylate) to Squalene as Core Material to Design Nanomedicines
}

Simona Mura, Elias Fattal, Julien Nicolas

\section{To cite this version:}

Simona Mura, Elias Fattal, Julien Nicolas. From Poly(Alkyl Cyanoacrylate) to Squalene as Core Material to Design Nanomedicines. Journal of Drug Targeting, 2019, 27 (5-6), pp.470-501. 10.1080/1061186X.2019.1579822 . hal-02323788

\section{HAL Id: hal-02323788 \\ https://hal.science/hal-02323788}

Submitted on 21 Oct 2019

HAL is a multi-disciplinary open access archive for the deposit and dissemination of scientific research documents, whether they are published or not. The documents may come from teaching and research institutions in France or abroad, or from public or private research centers.
L'archive ouverte pluridisciplinaire HAL, est destinée au dépôt et à la diffusion de documents scientifiques de niveau recherche, publiés ou non, émanant des établissements d'enseignement et de recherche français ou étrangers, des laboratoires publics ou privés. 


\title{
From Poly(Alkyl Cyanoacrylate) to Squalene as Core Material to Design Nanomedicines
}

\author{
Simona Mura, Elias Fattal, * Julien Nicolas
}

Institut Galien Paris-Sud, UMR 8612, CNRS, Univ. Paris-Sud, Université Paris- Saclay, Faculté de Pharmacie, 5 rue JB Clément, 92296 Châtenay-Malabry, France

*To whom correspondence should be addressed.

E-mail: elias.fattal@u-psud.fr

Tel:: +33146835582

Fax: +33146835511

\begin{abstract}
The discovery of biodegradable poly(alkyl cyanoacrylate) (PACA) nanoparticles by Patrick Couvreur has opened large perspectives in nanomedicine. Nanoparticles made from different types of PACA monomers have been used in different applications such as the treatment of intracellular infections or the treatment of multidrug resistant hepatocarcinoma. This latest application led to a Phase 3 clinical trial of Livatag ${ }^{\circledR}$, a PACA nanoparticulate formulation of doxorubicin. Despite the success of PACA nanoparticles, the need to develop novel type of nanoparticles with higher drug loadings and lower burst release was tackled by the discovery of squalene-based nanoparticles where the drug is covalently linked to the lipid derivative and the resulting conjugate self-assemble into nanoparticles. This pioneering work was accompanied by a wide range of novel applications which mainly dealt with the management of unmet medical needs (e.g., pancreatic cancer, brain ischemia and spinal cord injury). The present Review Article covers the most important steps of the pioneering work of Patrick Couvreur by trying to shed light on his outstanding career that has been a source of inspiration for many decades.
\end{abstract}

\section{Keywords}

Poly(alkyl cyanoacrylate), Nanoparticle, Squalene, Drug encapsulation, Prodrug, Nanomedicine 


\section{Introduction}

In 1975, Patrick Couvreur defended his PhD thesis at the Catholic University of Brussels under the supervision of Prof. Michel Roland. The subject of the doctoral dissertation was the disintegration mechanism of tablets containing starch [1, 2, 3]. In 1974, at the same University, Christian de Duve obtained the Nobel Prize in Medicine for his research over the structural and functional organization of cells. The discovery of the lysosome by De Duve inaugurated a new era in cellular physiology and pathophysiology which included, for instance the discovery of lysosomotropic agents. These discoveries inspired Patrick Couvreur who realized that, for major diseases (e.g., infectious diseases or cancer), there was a need to make the drug reach not only the right tissue but also the right cells and to do so, nanoscale systems would have been the best strategy. At the same time, a post-doctoral fellow in the group of Prof. Peter Speiser, a leader scientist in drug delivery working at the ETH in Zurich, described the first polymer nanoparticles (NPs) for drug delivery applications [4]. These nanoparticles, mainly used for the delivery of vaccines, were made of poly(methyl methacrylate) [5]. However, to safely deliver drugs intracellularly, the polymer had to be readily degradable to ensure excretion and avoid toxicity issues deriving from accumulation of foreign materials. From this observation arose the idea to develop poly(alkyl cyanoacrylate) nanoparticles (PACA) [6, 7]. The choice of this material was established as follows: (i) alkyl cyanoacrylate monomers can readily polymerize in aqueous medium which is of great interest for pharmaceutical purposes; (ii) PACA are biodegradable polymers, which is a prerequisite for intravascular administration and (iii) PACA-based surgical glues are widely used in humans and they exhibit low toxicity.

Polymer nanomedicines have been designed based on two parts: a core and shell. While the core is generally a biodegradable reservoir in which the drug is encapsulated, the shell has different cumulative functions, that were used to discriminate different generations of nanomedicines. While the first-generation nanoparticles are simply stabilized by surfactants to avoid aggregation and sedimentation, in the second generation the shell is made of grafted hydrophilic polymers that reduce the recognition by the monocyte phagocytic system (MPS). The third generation is obtained by functionalization of the shell with relevant ligands to allow cellular recognition.

Although exceptional potentialities have arisen from the use of polymer nanomedicines, including phase III clinical trials reached by doxorubicin-loaded PACA nanoparticles, there was still the need to develop new concepts in nanomedicine to circumvent their main limitations. This led Patrick Couvreur to move from the physical encapsulation of drugs, performed with PACA nanoparticles, to their chemical encapsulation, as shown with the recently-developed squalenoylation approach. Indeed, squalenoylation refers to a methodology which consists in coupling squalene, a natural lipid precursor of the cholesterol biosynthesis, to different biologically active molecules leading to squalene-based prodrugs able to self-assemble in aqueous medium into nanoparticles. 
The goal of the present review is to cover the main discoveries carried out by the group of Patrick Couvreur at the Catholic University of Louvain in Belgium and at the University of Paris-Sud in France, where he spent most of his career.

\section{Poly(alkyl cyanoacrylate) nanoparticles: from bench to clinical trials}

\section{Nanoparticle terminology and principle of the drug physical encapsulation}

Nanoparticles are submicronic colloidal systems with a mean diameter commonly ranging from 1 to $100 \mathrm{~nm}$, which can in many cases go up to $200 \mathrm{~nm}$. Most of them are spherical and denominated "nanospheres" or "nanocapsules", depending on their morphology [8]. Nanospheres are matrix system in which the drug is dispersed and/or adsorbed onto their surface, whereas nanocapsules are vesicular systems wherein the drug is contained in a liquid cavity serving as a reservoir surrounded by a polymeric shell [8]. The principle of physical encapsulation requires a material that can form nanoparticles after formulation and in which the drug can be physically entrapped. In the case of nanospheres, the drug to be encapsulated forms non-covalent bonds (strong ionic or weaker Van der Waals interactions) with the polymer matrix. In these conditions, it is usually very difficult to predict the amount of drug associated to the matrix. In the case of nanocapsules, the drug solubility in the core (water-containing or oil-containing) has been shown to be the limiting factor of the total drug that can be loaded $[9,10]$.

\section{Polymerization of alkyl cyanoacrylate monomers}

Alkyl cyanoacrylate monomers are known for their very high reactivity and the excellent adhesive properties of the resulting PACA polymers. For instance, the well-known Superglue ${ }^{\circledR}$ contains short alkyl chain cyanoacrylates whereas longer alkyl chain cyanoacrylates have been used for biomedical applications, the most famous examples being their use as surgical glue for the closure of skin wounds $[11,12,13,14,15,16,17]$ or as embolytic materials for endovascular surgery $[12,13,18]$. Because of their high reactivity, coming from the two electro-withdrawing groups (i.e., ester and cyano) in the $\alpha$ carbon of the double bond, alkyl cyanoacrylate monomers can polymerize extremely rapidly in the presence of nucleophiles such as anions (e.g., hydroxide, iodide, alcoholate) or weak bases (e.g., alcohol, amine), resulting in a very high polymerization kinetics.

PACA can be synthesized by either anionic (Figure 1a), zwitterionic (Figure 1b) or radical polymerization (Figure 1c). However, due to a faster reaction rate, anionic and zwitterionic polymerizations are greatly predominant compared to a radical mechanism. This explains why most studies on alkyl cyanoacrylates polymerization were mainly devoted to anionic and zwitterionic processes. 
It has been shown that the polymerization mechanism was governed by the experimental conditions. For instance, when simple anions (e.g., $\mathrm{CH}_{3} \mathrm{COO}^{-}, \mathrm{CN}^{-}, \mathrm{I}^{-}$) were used as initiators, ethyl cyanoacrylate (ECA) and $n \mathrm{BCA}$ polymerized via anionic polymerization whereas a zwitterionic mechanism was observed when using covalent organic bases (e.g., $\mathrm{Et}_{3} \mathrm{~N}$, pyridine) [19]. In particular for zwitterionic polymerization of $n$-butyl cyanoacrylate ( $n \mathrm{BCA}$ ), it was shown that nature of the initiator (e.g., phosphine, pyridine and amine derivatives) [20, 21, 22, 23, 24, 25, 26], the presence of inhibitors or water, had a strong influence on the macromolecular characteristics of the polymer and the polymerization kinetics. As for anionic polymerization, when tetrabutyl ammonium salts (hydroxide, bromide, acetate and substituted acetates) were used as initiators for the polymerization of $n \mathrm{BCA}$, a quasi-ideal living polymerization was reported for hydroxide-based initiators [27, 28, 29]. When a suitable inhibitor (e.g., boron trifluoride-acetic acid complex, acetic acid, propane-1,3-sultone) is introduced in the reaction medium, free-radical polymerization is predominant over ionic polymerizations $[30,31,32,33,34]$ and can be initiated by conventional radical initiators (e.g., azobisisobutyronitrile, benzoyl peroxide).

(a)<smiles>[R]OC(=O)C(C)(C)CC(C)(C(=O)O[R])C(=O)O[R]</smiles>

(b)<smiles>[R]OC(=C)/C(C#N)=C/CCCC[N]</smiles><smiles>[R]OC(=O)C(C)(C)CC(C)(C[C@H](C)N)C(=O)O[R]</smiles>

(c)<smiles>[R]OC(=O)C(=C)CCCCCP</smiles><smiles>[R]OC(=O)/C(C#N)=C/CCCCC(C)(CP)C(=O)O[R]</smiles><smiles>C#CC</smiles><smiles>[R]OC(=O)C1(CC(C)P)CCC1C#N</smiles>

Figure 1. Initiation and propagation steps involved during (a) anionic, (b) zwitterionic and (c) radical polymerization of alkyl cyanoacrylate monomer initiated by a base (B-), a nucleophile ( $\mathrm{Nu})$ and a radical $(\mathrm{P} \bullet)$, respectively. 
Interestingly, alkyl cyanoacrylate can also be copolymerized by free-radical copolymerization with other vinyl monomers such as methyl methacrylate (MMA) or styrene, leading to random or alternating copolymers, respectively.

\section{Preparation of PACA nanoparticles}

PACA nanoparticles can be prepared by polymerization of alkyl cyanoacrylate monomers or by formulation of preformed PACA polymers. Polymerization methods gives access to either nanospheres or nanocapsules. First examples of PACA nanospheres were prepared by anionic emulsion polymerization at low $\mathrm{pH}(\sim 2-3)$ initiated by bases present in the medium, such as $\mathrm{OH}$ - ions deriving from water dissociation [35, 36]. As for nanocapsules, Al Khouri-Fallouh et al. [9] proposed an original method in which the monomer was solubilized in an oil-containing alcohol phase followed by dispersion in an aqueous phase containing surfactants. In contact with water, the alcohol phase diffused and favored the formation of a very fine oil-in-water emulsion, where polymerization initiated by $\mathrm{OH}$ ions took place at the monomer droplet/continuous water phase interface. This simple process was successfully applied to the encapsulation of large quantities of lipophilic drugs. Other authors have suggested systems adapted to hydrophilic molecules in which the monomer polymerizes at the monomer droplet/continuous oily phase interface to form the nanocapsule shell [10, 37]. For intravenous administration, aqueous core-containing nanocapsules were transferred from the oily phase into an aqueous continuous phase by ultracentrifugation of the oily suspension over a layer of pure water containing a non-ionic surfactant $[10,37]$.

PACA nanoparticles can also be obtained from preformed polymer. The main advantage of this approach is that nanoparticles are made from well-characterized and purified polymers. Also, their intrinsic physico-chemical characteristics will not depend on the conditions encountered during the nanoparticle preparation as it can be the case with the previously described methods. Based on the solubility properties of a polymer, the general principle is to prepare a solution of the polymer and to induce a phase separation by the addition of a polymer non-solvent [38]. Phase separations leading to polymer nanoparticles are usually obtained with diluted solutions of polymers. In this method, polymers such as PACA are dissolved in a water-miscible solvent (e.g., acetone) and the polymer solution is added into water. The acetone diffuses into water, causing the formation of the nanoparticles, and is eventually evaporated [39]. Surface active agents are usually added to water to ensure the stability of the polymer nanoparticles [39]. Nanocapsules can easily be prepared by the same method just by adding a small amount of an organic oil in the polymer solution. When the latter is poured into the water phase, the oil is dispersed as tiny droplets in the solvent/non-solvent mixture and the polymer precipitates at the oil/droplet surface. This method led to the preparation of oilcontaining nanocapsules and was valuably used for the encapsulation of liposoluble drugs.

\section{Physico-chemical properties of PACA nanoparticles}


PACA nanospheres prepared by emulsion polymerization have an average size around $200 \mathrm{~nm}$ that can be reduced to $30-40 \mathrm{~nm}$ by addition of a nonionic surfactant in the polymerization medium [40] or $\mathrm{SO}_{2}$ to the monomer [41]. Entrapment efficiency, that is the percentage of drug present within the nanospheres compared to the initial amount of drug, has been shown to vary consistently with polymer physico-chemical properties, drug nature, and preparation method. It was also strongly dependent on the monomer alkyl chain length as well as the drug physico-chemical characteristics. Drugs can be entrapped into PACA nanoparticles during the polymerization process by dissolution in the polymerization medium or can be adsorbed onto nanoparticles surface after their preparation. Generally, the drug loading, that is the weight fraction of drug with respect to the total nanoparticle weight (i.e., drug + polymer) is higher when drug is incorporated during the polymerization reaction. Hydrophilic drugs, such as ampicillin and doxorubicin, can be entrapped with a high loading [42, 43]. High molecular weight substances, such as monoclonal antibodies, insulin or growth hormone releasing factor (GRF), can also be entrapped with a very high loading efficiency [44, 45, 46]. Interestingly, some drugs such as vidarabine [47], can initiate the polymerization of alkyl cyanoacrylate, leading to a covalent bond between the polymer and the drug, and hence a decrease in the drug activity since the drug is not released from the conjugate here formed non intentionally [48].

The drug release kinetics of PACA nanoparticles depends on whether the drug has been incorporated into the nanoparticle or simply adsorbed on its surface. The drug release, after either encapsulation and adsorption, is biphasic, with an initial fast release phase followed by a much slower second one. The initial release rate is faster when the drug is located onto the surface than when it is incorporated within the polymer matrix. The release rate generally decreases with the polymer molar mass, the particle size and the drug physico-chemical characteristics and eventual interactions with the polymer. Grangier et al. suggested that drug release from nanoparticles resulted from polymer bioerosion [45] rather than diffusion through the polymer matrix, as shown by Lenaerts et al. [49], who correlated drug release with polymer degradation, explaining the influence of alkyl chain length on modulation of drug release [50]. These results were supported by the observation that the drug release increased in presence of esterase in the medium $[42,45]$. Thus, preparing nanoparticles from different PACA polymers in different proportion allowed to modulate the drug release [50].

Oil-containing PACA nanocapsules have been developed to encapsulate lipophilic molecules such as indomethacin [51]. Interestingly, they have also been extensively used to encapsulate hydrophilic drugs such as peptides, including very large ones such as insulin [52,53] and calcitonin [54], which were entrapped at very high encapsulation efficiency compared to smaller peptides [55]. The encapsulation efficiency of small lipophilic drugs was shown to be related to their solubility, while for peptides it seemed to depend on other parameters such as their larger molecular weight. The extremely rapid polymerization of alkyl cyanoacrylate monomers occurring at the surface of the oil droplet limited the diffusion of the peptide towards the aqueous phase leading to its entrapment into the nanocapsules. This mechanism is consistent with the fact that the encapsulation is more efficient with 
higher molecular weight peptides since they are less likely to escape from the oil cavity and diffuse through the polymer film. In contrast to what has been observed with PACA nanospheres, peptides do not chemically react with the alkyl cyanoacrylate monomers during the preparation of nanocapsules. The presence of a large excess of alcohol likely prevented peptide hydroxyl and amino groups from initiating the polymerization, thus preserving their biological properties.

Water-containing PACA nanocapsules were also designed by the Couvreur's group. In general, the process led to nanocapsules with diameters ranging from 250 to $350 \mathrm{~nm}$. There were designed to encapsulate hydrophilic molecules such as antisense oligonucleotides (Figure 2) [10,37] and small interfering RNA [56] with high encapsulation yields as well. The internal localization of nucleic acids induced a better protection against nucleases compared to a simple adsorption onto cationic PACA nanospheres. Successful encapsulation of nucleosides was also achieved in such nanocapsules by the coencapsulation of polycations forming an ion pair with phosphate nucleosides [57].

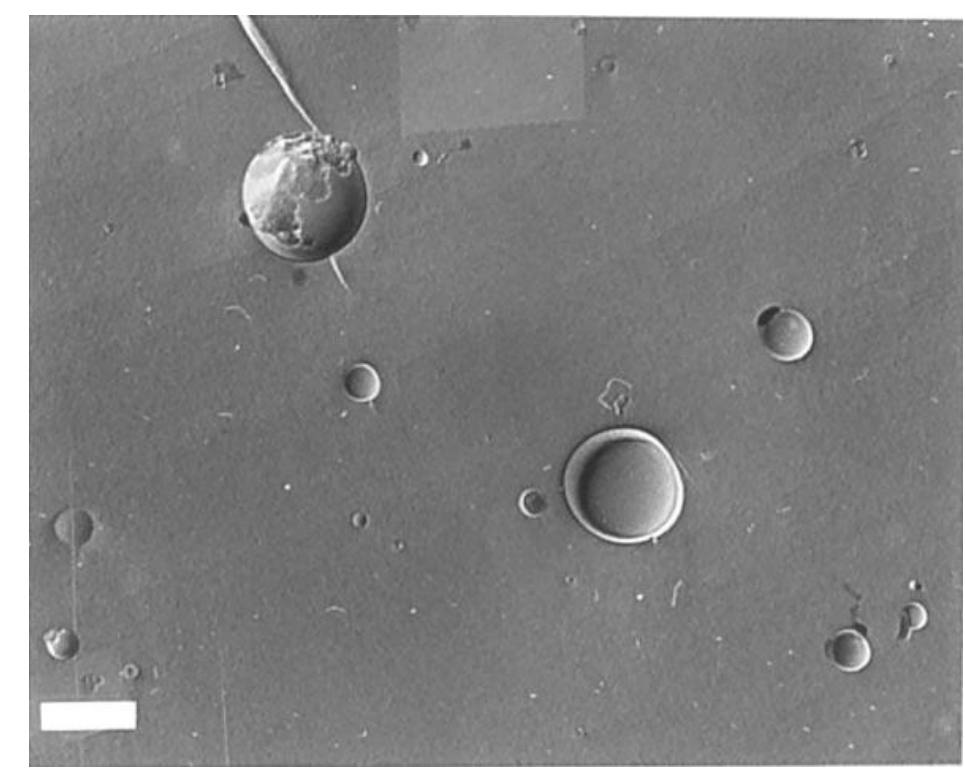

Figure 2. Freeze fracture electron microscopy of water-containing PACA nanocapsules. Scale bar = $500 \mathrm{~nm}$. Reproduced with permission from Ref. [37].

\section{Biological fate of PACA nanoparticles and their drug content}

Very early after designing PACA nanospheres, a study related to their in vivo fate was conducted. Their intravenous injection in mice and rats demonstrated that they were likely opsonized by plasma proteins and recognized by the MPS, resulting in their rapid clearance from the blood stream [58]. Autoradiographic studies have evidenced that nanoparticles were mainly concentrated in the liver, the spleen and the bone marrow [58]. The liver content in PACA nanospheres gradually decreased and, after being metabolized, the carrier was excreted via the feces and urine [58] and no physical blockade of Kupffer cell phagocytic function occurred in the long term [59]. Excretion of the nanoparticles was complete after 7 days. The situation can be summarized by suggesting that the liver acts as a reservoir 
of nanoparticles, conditioning their rapid first-phase disappearance from the blood and their secondphase release in the body under degraded and excretable forms. Since most nanoparticles were captured by the liver after intravenous administration, the intrahepatic distribution of PACA nanoparticles was investigated. Lenaerts demonstrated that Kupffer cells were the major liver site of accumulation of PACA nanospheres [60], while a very low uptake was observed by endothelial and especially parenchymal cells. Uptake of nanoparticles by Kupffer cells was consistent with in vitro experiments carried out with macrophages [61, 62] or fibroblasts [63] which showed that endocytosis played a major role in the uptake process. After being endocytosed, PACA nanospheres were located within the lysosomes $[62,64]$. Biodegradation through a bioerosion process occurred in this cellular compartment by means of esterases. During degradation, the hydrocarbon chain of the polymer remains unchanged while the enzymatic cleavage of lateral ester groups induced the solubilization of the resulting poly(cyano acrylic acid) polymer, leading to a progressive disassembly of the nanoparticles into phagolysosomes [49]. After loading into PACA nanoparticles, the fate of the drug, its tissue distribution and pharmacokinetic were significantly modified [65]. Since the drug distribution is generally correlated with the distribution profile of the carrier itself, it is admitted that the drug release does not occur in the blood stream after intravenous administration, because of the rapid elimination of the carriers. It was also shown at the same period that alteration of the drug distribution profile by encapsulation of some anticancer drugs into nanoparticles considerably reduced the toxicity of the drug. This phenomenon was attributed to the reduced accumulation of drug in organs where most acute toxic effects are exerted [66].

The modulation of the uptake of PACA nanospheres by MPS was made feasible through their surface coating with hydrophilic and flexible polymers such as poly(ethylene glycol) (PEG) or polysaccharides. Even though simple adsorption of PEG-based surfactants like poloxamers was envisioned it has been demonstrated, for instance from PACA nanoparticles on which poloxamer 388 or poloxamine 908 was adsorbed, that surfactant desorption after in vivo administration resulted in similar biodistribution than uncoated nanoparticles [84]. To circumvent this limitation, grafting PEG chains at the surface of PACA nanospheres was achieved in situ during anionic/zwitterionic emulsion polymerization using ability of PEG to act as initiator and therefore create a covalent bond with PACA chains [67, 68]. Similarly, polysaccharides (e.g., dextran, chitosan, etc.) were also used as stabilizing/initiating moieties to give surface-modified PACA nanospheres [69, 70]. Chauvierre et al. then adapted Couvreur's original protocol to a free-radical emulsion polymerization using a polysaccharide/Ce4+ couple as the initiator [71, 72]. Various polysaccharides were used and exhibited different conformation $[69,73,74]$ (e.g., brush, loop) at the surface of the PACA nanoparticles that impacted both complement activation (Figure 3) [70] and cytotoxicity [75].

Among the different approaches to obtain PEGylated PACA nanoparticles from preformed polymers, the synthesis of poly[(hexadecyl cyanoacrylate)-co-methoxypoly(ethylene glycol) cyanoacrylate] 
(P(HDCA-co-MePEGCA)) [76] was certainly the most promising one [77, 78] as protein adsorption considerably decreased when compared to non-PEGylated counterparts [79]. Also, liver uptake was reduced and the circulation time significantly increased in a mice model [80]. Interestingly, P(HDCAco-MePEGCA) [76] nanoparticles exhibited favorable and somewhat unexpected properties for the passage of the blood-brain barrier (BBB) compared to the non-PEGylated counterparts and NPs with pre-adsorbed PEG-based surfactants (Figure 4) [81, 82, 83, 84].

A

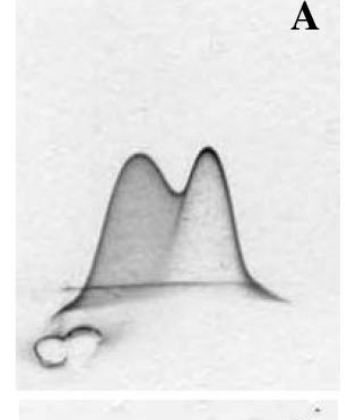

D

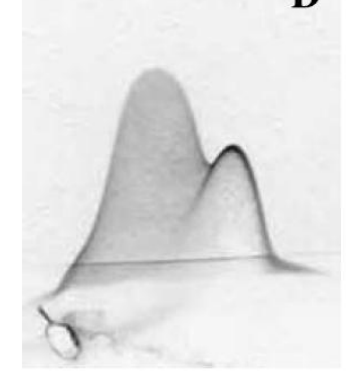

B

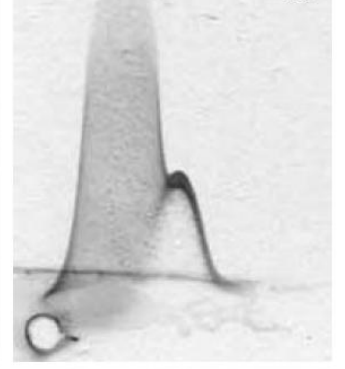

$\mathbf{E}$

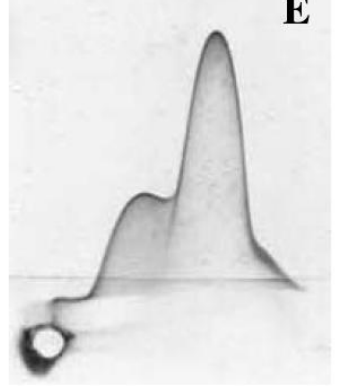

C
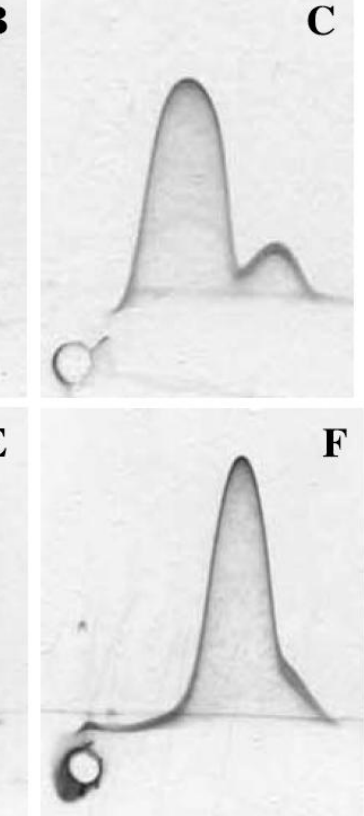

Figure 3. Complement activation profiles of NPs prepared via $(A, B, C)$ free-radical emulsion polymerization or (D, E, F) anionic emulsion polymerization at $\mathrm{pH} 2.5$ from (A, D) $10 \mathrm{kDa}$ dextran, (B, E) $67 \mathrm{kDa}$ dextran, or (C, F) $100 \mathrm{kDa}$ dextran. Reproduced with permission from Ref. [70].

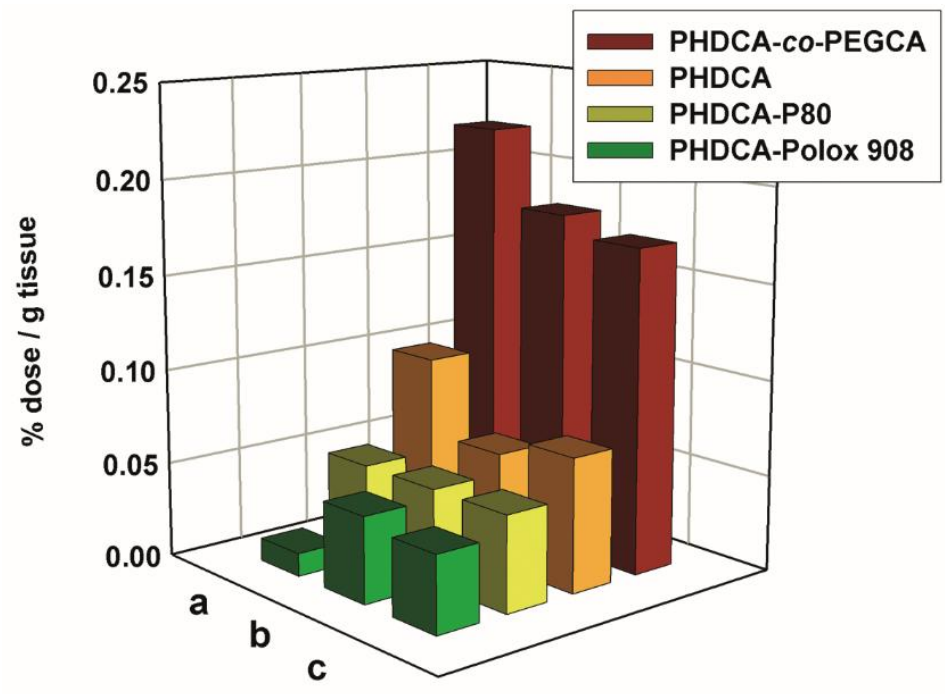


Figure 4. Concentration of radioactivity in (a) right hemisphere, (b) left hemisphere and (c) cerebellum or mice $1 \mathrm{~h}$ after IV administration of $\left[{ }^{14} \mathrm{C}\right]-\mathrm{P}(\mathrm{HDCA}-\mathrm{co}$-MePEGCA) NPs, poloxamine 908-coated $\left[{ }^{14} \mathrm{C}\right]$-PHDCA NPs, polysorbate 80 -coated $\left[{ }^{14} \mathrm{C}\right]$-PHDCA NPs, and uncoated $\left[{ }^{14} \mathrm{C}\right]$-PHDCA NPs. Adapted with permission from Ref. [7].

The identified mechanism by which $\mathrm{P}(\mathrm{HDCA}-$ co-MePEGCA) nanoparticles preferentially crossed the healthy BBB relied on a specific adsorption of apolipoprotein E and B-100 onto their surface which allowed their translocation via low-density lipoprotein receptors (LDLR). It was also shown that PEG chains exposed at the surface of P(HDCA-co-MePEGCA) nanoparticles had significant affinity for the $\mathrm{A} \beta_{1-42}$ amyloid peptide, which is a well-known biomarker of the Alzheimer's disease (AD) [85, 86, 87]. Stealth $\mathrm{P}(\mathrm{HDCA}-\mathrm{co}$-MePEGCA) nanoparticles were also made fluorescent by incorporation of rhodamine moieties in the copolymer structure [88] or by encapsulation of quantum dots [89], leading to useful imaging tools for the accurate determination of the fate of the nanoparticles either in vitro or in vivo.

\section{PACA nanoparticles for active targeting}

The synthesis of ligand-decorated PACA nanoparticles for achieving specific cells targeting on the basis of molecular recognition has been the topic of intensive research on the group of Patrick Couvreur. The most efficient approach relied on the coupling of biologically ligands at the extremity of PEG chains so that they would be displayed at the surface of nanoparticles. Depending on the size of the ligand, the coupling may be performed either before nanoparticle formulation (small size ligands) or after nanoparticle formulation (both small- and large-size ligands), for obvious physico-chemical and self-assembly reasons. This strategy was first investigated by Stella et al. [90] who formulated folic acid-functionalized, PEGylated PACA nanoparticles. They first prepared P(HDCA-co$\mathrm{H}_{2}$ NPEGCA) nanoparticles that were further functionalized with folic acid by carbodiimide coupling chemistry. (Figure 5) These nanoparticles were able target folate receptor over-expressing cancer cells [90, 91], thus opening up new perspectives for such so-called third generation PACA nanoparticles. 

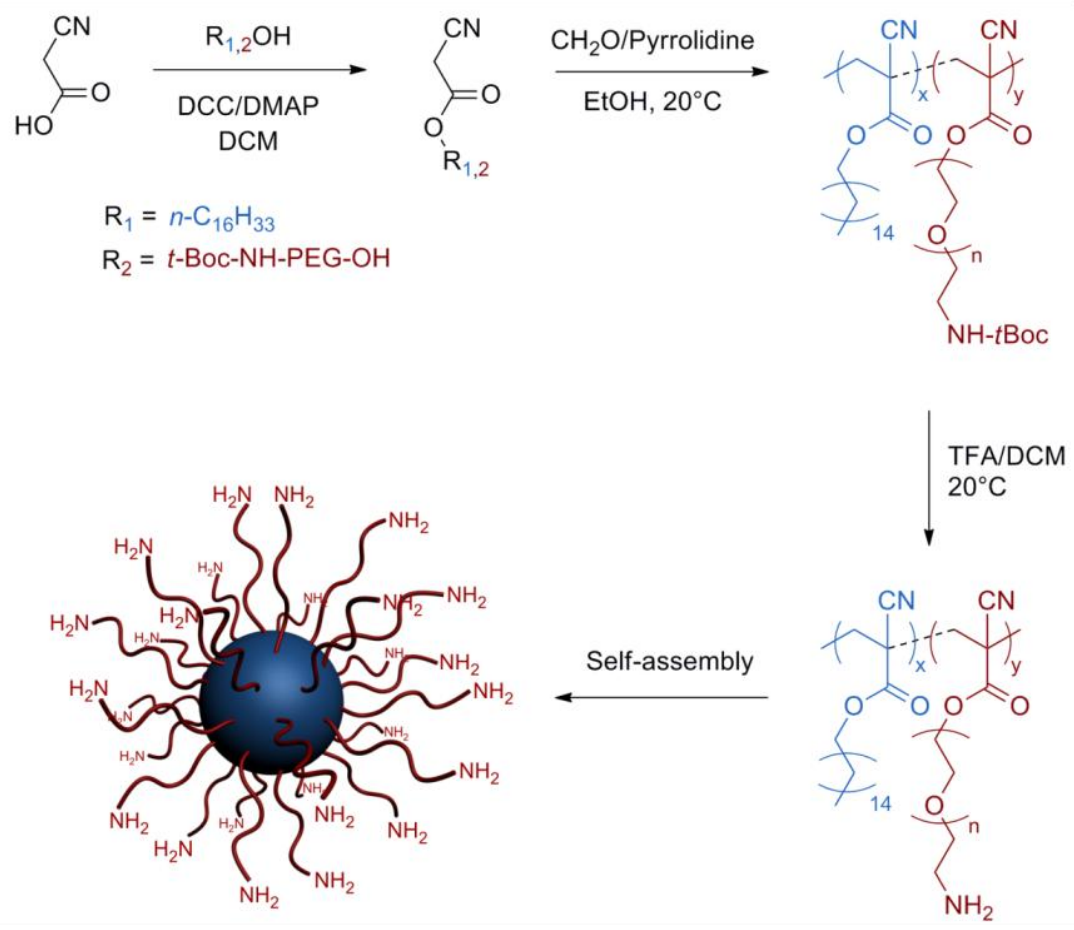

Figure 5. Synthesis of $\mathrm{P}\left(\mathrm{HDCA}-\mathrm{co}-\mathrm{NH}_{2} \mathrm{PEGCA}\right)$ copolymer for further $\mathrm{NP}$ surface functionalization. Adapted with permission from ref. [92].

A versatile nanoparticulate platform based on PACA, exhibiting stealth, fluorescent and targeting properties was reported by the group of Couvreur [93]. Targeted PACA nanoparticles were obtained by means of "click chemistry" which is a powerful orthogonal ligation strategy [94]. The ligand of interest was linked to P(HDCA-co-PEGCA) copolymer prior formulation into nanoparticles. The versatility of the synthetic route was illustrated by the coupling of a small library of ligands such as biotin, selegiline and curcumin derivatives to efficiently and selectively target cancer cells in vitro (biotin, [93] with enhanced cytotoxicity when paclitaxel was encapsulated), or the $A \beta_{1-42}$ peptide (selegiline, [95] curcumin derivatives [93]). The different ligands were previously derivatized with an alkyne group (except selegiline that already had one in its structure) (Figure 6). 


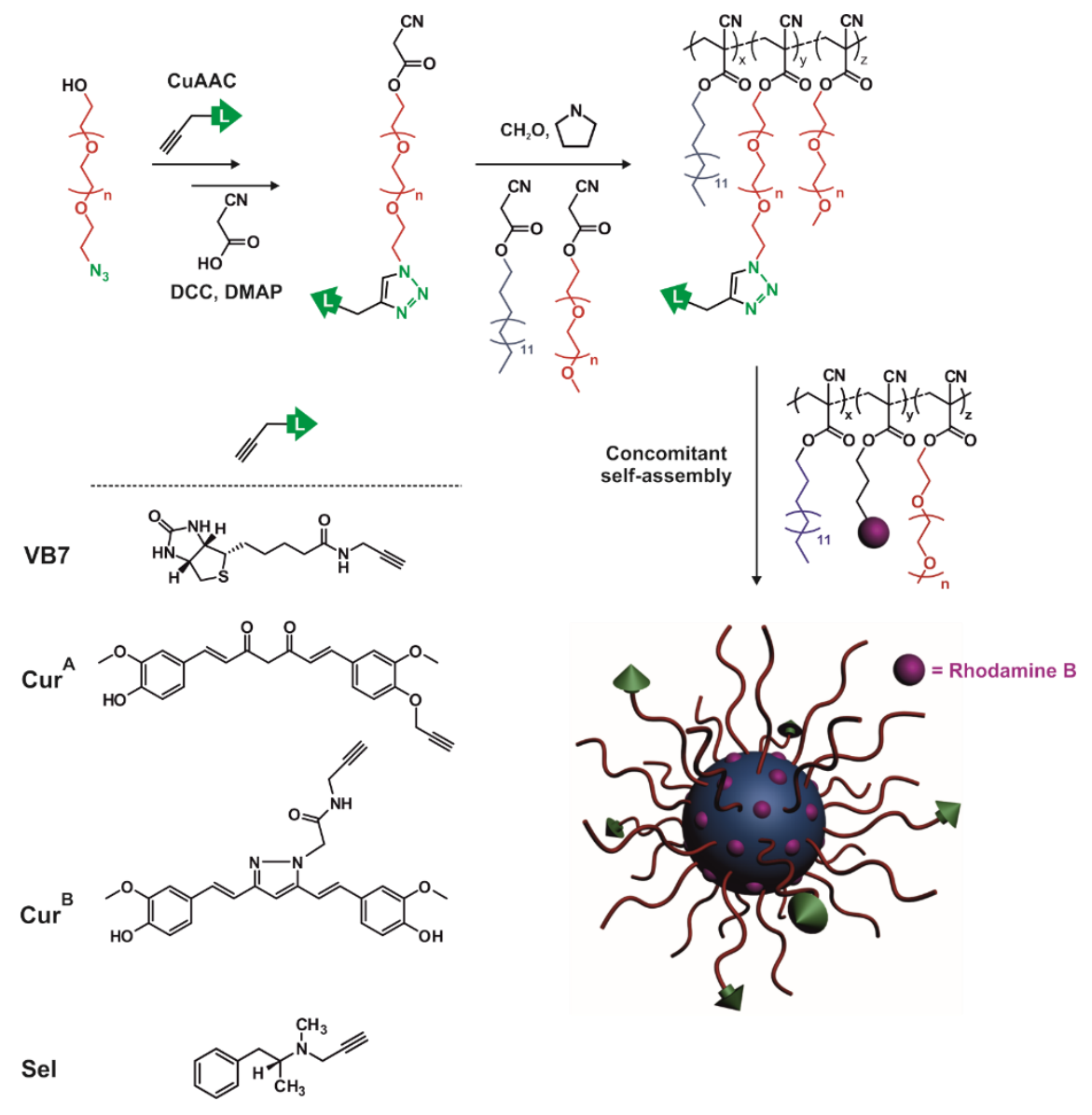

Figure 6. Synthesis of fluorescent, PEGylated and biodegradable P(HDCA-co-PEGCA) nanoparticles functionalized with biotin (VB7), selegiline (Sel) or curcuminoid derivatives (CurA and CurB). Adapted with permission from ref. [92].

Remarkably, the presence of biotin at the surface of PACA nanoparticles was also used to anchor monoclonal anti-A $\beta_{1-42}$ antibodies after incubation of the nanoparticles with streptavidin-anti-A $\beta_{1-42}$ through avidin/biotin ligation strategy [93]. It was shown a few years later that treatment of AD-like transgenic mice with anti-A $\beta_{1-42}$-functionalized PACA nanoparticles led to complete correction of the memory defect, significant reduction of the $\mathrm{A} \beta$ soluble peptide and its oligomer level in the brain, and significant increase of the $A \beta$ levels in plasma[96].

\section{Major applications of PACA nanoparticles by parenteral administration}

Different applications of PACA nanoparticles have been considered by the Couvreur's group. Among them, the most important dealt with the treatment of intracellular infections, anticancer therapy (including targeting of nucleic acids), brain delivery, and finally oral or controlled delivery of peptides and proteins. 


\subsection{PACA nanoparticles for the treatment of intracellular infections}

A great deal of work has been focused on the use of PACA nanospheres for drug delivery in the management of intracellular infections. Indeed, infected cells may constitute a "reservoir" for microorganisms whose accumulation inside the lysosomes protect them from antibiotics. The resistance of intracellular infections to chemotherapy is often related to the low uptake of commonly used antibiotics or to their reduced activity at the acidic $\mathrm{pH}$ of lysosomes. To overcome these drawbacks, the use of antibiotic-loaded PACA nanospheres was proposed as endocytozable formulation [97]. The preparation of ampicillin-loaded, biodegradable nanospheres, either poly(isobutyl cyanoacrylate) (PIBCA) or poly(isohexyl cyanoacrylate) (PIHCA), was achieved and the antimicrobial activity of the drug remained unaltered upon loading into the carrier [42, 98]. The effectiveness of PIHCA nanospheres was tested in the treatment of two experimental models of intracellular infections. Ampicillin-loaded nanospheres were first administered in the treatment of experimental Listeria monocytogenes infection in congenitally athymic nude mice, a model involving a chronic infection of both liver and spleen macrophages [99]. After adsorption onto nanospheres, the therapeutic activity of ampicillin was found to increase dramatically over that of the free drug. Bacterial counts in the liver were at least 20-fold diminished after loading ampicillin into PIHCA nanospheres. In addition, ampicillin-loaded PIHCA nanospheres ensured liver sterilization after two injections of $0.8 \mathrm{mg}$ whereas no effect was observed with any of other regimens tested.

Efficacy of ampicillin-loaded nanospheres was then evaluated in the treatment of experimental salmonellosis in C57/BL6 mice, a model involving an acute fatal infection [97]. All mice treated with a single injection of ampicillin-loaded nanospheres survived whereas in control and empty nanospheregroups, all mice died within 10 days post infection. To be noted that with free ampicillin an effectivecurative effect required 3 doses of $32 \mathrm{mg}$ each. Lower doses ( 3 x $0.8 \mathrm{mg}$ and $3 \times 16 \mathrm{mg}$ ) delayed but did not reduce mortality. Thus, the therapeutic index of ampicillin, calculated according to mice mortality, was increased by 120 -fold when the drug was loaded into nanospheres.

To clarify the mechanism by which nanospheres improved the antimicrobial efficacy of ampicillin, Forestier et al. [100] compared in vitro the efficacy of ampicillin loaded into PIBCA nanospheres with that of free ampicillin in terms of survival of L. monocytogenes in mouse peritoneal macrophages. After $30 \mathrm{~h}$ of incubation, nanospheres decreased the number of viable bacteria by $99 \%$ as compared to control treatments whereas only slight differences were observed between free ampicillin and controls. Ampicillin-loaded nanoparticle thus appeared to be much more effective than free drug for inhibiting intracellular growth of L. monocytogenes. A more complicated situation was observed with in vitro S. typhimurium-infected macrophages, since the bactericidal effect of ampicillinloaded PIHCA nanospheres was poor in spite of the dramatic increase of the intracellular capture of ampicillin and the reduction of its efflux in the extracellular medium [101]. In another study, confocal microscopy and transmission electron microscopy were used to establish the intracellular trafficking of ampicillin-loaded PIHCA nanospheres and their colocalization with the bacteria within the subcellular 
compartments [62]. The active uptake by phagocytosis of ampicillin-loaded PIHCA nanospheres by murine macrophages was clearly demonstrated together with their localization in the same vacuoles as the infecting bacteria [62]. Such a limited bactericidal effect of the nanospheres was therefore quite puzzling. The most probable explanation was to be found in the resistance mechanism of $S$. typhimurium involving the inhibition of the phagosome-lysosome fusion [102] which allowed the survival of the bacteria accumulating in the nanosphere-free phagosomes.

\subsection{PACA nanoparticles for anticancer therapy}

One of the most extensive research of Patrick Couvreur focused on the use of PACA NPs for the delivery of anticancer drugs. Dactinomycin-loaded poly(methyl cyanoacrylate) (PMCA) nanospheres were first developed in Brussels and tested against soft tissue sarcoma S 250, an experimental solid tumor of the rat [103]. This first experiment demonstrated a marked tumor growth inhibition in rats treated with dactinomycin-loaded PMCA nanospheres compared to the free dactinomycin. However, rat survival curves showed that the encapsulated drug form was more toxic than the free drug. Significant reduction of toxicity was achieved by using poly(hexyl cyanoacrylate) (PHCA), a less histotoxic structural homologue. Doxorubicin-loaded PIBCA nanoparticles [66] were then designed just before moving to the University of Paris-Sud. The first experiment evidenced that when the drug was loaded into nanospheres, significant reduction of both mortality and weight loss of healthy mice were recorded under various administration schedules. Furthermore, cardiotoxicity was decreased due to the poor uptake by the myocardium [66]. Since liver was shown to be one of the main site of nanoparticle uptake, a study was carried out on a sarcoma M 5076 model of liver metastases demonstrating a better efficiency of doxorubicin-loaded nanospheres due to their important capture by the Kupffer cells [104]. This effect likely led to the formation of an effective gradient of concentration favorable for a massive and prolonged diffusion of the drug towards the neighboring malignant cells. Therefore, and irrespective to the dose and the administration regime, the reduction of the number of metastases was far much larger with doxorubicin-loaded nanospheres than with the free drug [104]. The superiority of the nanoparticulate system was clearly evidenced by histological examinations, showing that both the number and the size of the tumoral cores were lower when doxorubicin was loaded into nanospheres.

The problem of cancer multidrug resistance (MDR), which is the main cause of chemotherapy failure, was then investigated using PIHCA nanospheres [105]. MDR is often associated with the overexpression of a 170-kDa cell membrane glycoprotein (i.e., P-glycoprotein (P-gp)) [106, 107], which could act as an efflux pump and clear numerous drugs from the cells as also shown for bacterial transport proteins $[106,107,108]$. Indeed, multidrug resistance is associated with a low intracellular accumulation of some drugs. To solve this problem, the efficacy of doxorubicin-loaded nanospheres was evaluated by Cuvier et al. [105] on five different multidrug resistant cell lines, whose mechanism of pleiotropic resistance was known to be related to the presence of P-glycoprotein. A complete 
reversion of drug resistance was obtained in vitro, which resulted in a cell growth inhibition comparable to that obtained with sensitive cells exposed to free doxorubicin. Similar results were obtained by Kubiak et al. [109] on the resistant DC3F-AD/AZA subline. It was thus made clear that in vitro resistance of tumor cells to doxorubicin can be fully circumvented using biodegradable PIHCA nanospheres. Doxorubicin-loaded nanospheres were also tested in doxorubicin-resistant C6 rat glioblastoma cell lines differing by their degree and mechanism of resistance [110]. The key finding of this study was that reversal of doxorubicin resistance by nanospheres was closely dependent on the nature of this resistance: nanospheres were only efficient on pure MDR phenotype cells and not on the additional mechanism of resistance to doxorubicin [110]. The mechanism behind this important observation was not fully understood although it was found that PIBCA nanospheres allowed higher intracellular concentrations of doxorubicin to be reached, which was correlated with a higher cytotoxicity compared to the free drug [111]. However, the lack of influence of cytochalasin B on the cellular drug uptake suggested that endocytosis was not the main mechanism of nanoparticle internalization [112]. According to the increase of intracellular drug accumulation, it was hypothesized that the rapid drug release of doxorubicin from nanospheres adhering to the cell membrane induced an overflow and saturation of P-gp [112]. However, the most probable explanation relies on the formation of an ion-pair association of doxorubicin with soluble degradation products of PACA which, conversely to free doxorubicin, were no longer a substrate for P-gp [113]. Doxorubicin-loaded PIHCA nanospheres were investigated in vivo in a model of hepatocarcinoma in transgenic mice overexpressing MDR genes, demonstrating a greater antitumor drug efficacy compared to the free drug [114].

A phase I clinical trial has been conducted in 21 patients with refractory solid tumors using these nanospheres. In this study [115], granulocytopenia appeared to be the dose-limiting toxicity. However, the comparison of this toxicity with historical data relative to free doxorubicin suggested that the nanospheres were, in some respects, less myelotoxic. Interestingly, no cardiotoxicity was noticed using Doppler-Echocardiography among 18 evaluable patients. Also, the lack of hepatic toxicity ruled out potential risk of liver damage following doxorubicin accumulation from nanoparticle capture by hepatic Kupffer cells. However, appearance of an allergy shortly after the beginning of the infusion (15 $\mathrm{mg} / \mathrm{m}^{2}$ ) in 2 patients at, without any history of allergic reactions, was an unexpected and a disturbing event [115]. This complication was almost circumvented by NP dilution in $5 \%$ dextrose and increasing of the perfusion duration up to $60 \mathrm{~min}$. This phase 1 study was completed around 15 years later by another phase 1-2 clinical trial in advanced hepatocellular carcinoma. It was carried out by the company BioAlliance Pharma ${ }^{\circledR}$ (now Onxeo ${ }^{\circledR}$ ) who gave the name of Livatag ${ }^{\circledR}$ to the drug. In phase 1, hematological and respiratory limited toxicities were reported at 35 and $40 \mathrm{mg} / \mathrm{m}^{2}$, giving a MTD at $30 \mathrm{mg} / \mathrm{m}^{2}$ [116]. Partial response rate was $10 \%$, and stable disease $70 \%$. The phase 2-3 trial presented preliminary survival results arising from patients with unresectable hepatocellular carcinoma (primary liver cancer). Out of the total 50 patients planned, 28 were randomized and received intra-arterial 
injection of $\operatorname{Livatag}^{\circledR}(\mathrm{n}=17)$ or chemoembolization $(\mathrm{n}=11)$, according to a $2 / 1$ scheme $(2$ patients with Livatag ${ }^{\circledR}$ for 1 patient with chemoembolization). Livatag ${ }^{\circledR}$ was administered every 4 weeks, up to 3 injections. Although the trial had been put on hold due to severe pulmonary adverse events, the assessment of survival has been continued based upon the recommendation of the independent data safety monitoring board. The survival results for patients treated with Livatag ${ }^{\circledR}$ showed a significant increase compared to the control group with 31.7 months median survival versus 15 months. Patients who received 3 injections had an even better response with an increased median survival (33 versus 15 months) [116]. The phase 3 is still ongoing but Onxeo ${ }^{\circledR}$ recently announced that the study did not meet its primary endpoint of improving survival over the comparative group. Indeed, since the study was not placebo-controlled, patients in the comparative group received other anticancer agents (e.g., oxaliplatin, gemcitabine or tyrosine kinase inhibitors) which might explain the high survival rate of the control arm. Livatag ${ }^{\circledR}$, as single agent, showed a similar effect as the one observed in the comparative group with active treatments. There was no difference in efficacy between the two arms (Livatag ${ }^{\circledR} 20$ $\mathrm{mg} / \mathrm{m}^{2}$ and $30 \mathrm{mg} / \mathrm{m}^{2}$ ). Nevertheless, the monitoring of the patients still enrolled in the study will continue to completion expected in 2019.

In the early 90's, the group oriented some research on the application of PACA for nucleic acid delivery. The first molecules to be delivered were antisense oligonucleotides (ASO) followed by small interfering RNA (siRNA). ASO and siRNA are potentially powerful new drugs because of their selective inhibition of gene expression. They are therefore very potent in the treatment of cancer mainly because of their capacity to target specific signaling pathways. However, their use as therapeutics is a challenge because of their high susceptibility to enzymatic degradation and their poor penetration across biological membranes. Their incorporation into nanoparticulate systems has appeared as an interesting alternative that would enhance their stability in biological fluids. In the case of nanospheres made of synthetic polymers, since oligonucleotides have no affinity for the polymeric matrix, association with nanoparticles has been achieved by ion pairing with a cationic surfactant (e.g., cetyltimethylammonium bromide (CTAB)) adsorbed onto the nanoparticle surface [117]. Oligonucleotides bound to PACA nanospheres in this way were protected from nucleases in vitro [118] and their intracellular uptake was increased [119]. In addition, nanospheres were able to concentrate intact oligonucleotides in the liver and in the spleen [120]. This formulation strategy also allowed ASO to specifically inhibit cell proliferation and tumorigenicity, mediated by mutated Ha-ras, in nude mice [121].

However, these nanospheres had two drawbacks which were their toxicity, mainly due to the presence of CTAB [122], and the quick desorption of the ASO in the presence of serum, which resulted from nanoparticle surface erosion by serum esterases [120]. Accordingly, PACA nanocapsules with an aqueous core containing the ASO were developed. Stability studies demonstrated that nanoencapsulation was able to protect ASO against degradation by serum nucleases with a higher efficacy compared to CTAB-coated nanospheres, in which the oligonucleotides were 
simply adsorbed onto the NP surface $[118,119]$ rather than encapsulated. Phosphorothioate ASO directed against the fusion gene EWS-Fli1 responsible of Ewing sarcoma were encapsulated within PACA nanocapsules and tested in vivo for their efficacy against the tumor model in mice after intratumoral administration [123]. Only intratumoral injection of ASO-loaded nanocapsules led to a significant inhibition of tumor growth. No antisense effect could be detected with the free oligonucleotide. In a previous study, using the same antisense sequence as a free drug, Tanaka et al. [124] demonstrated inhibition of a tumor growth in a similar model, but a cumulative dose of 500 nmol oligonucleotide was needed. With nanocapsules it was possible to obtain an comparable effect to that observed by these authors but with a 35-fold lower dose. Therefore, the PACA nanocapsule technology allowed to lower phosphorothioate doses and thus avoided the toxicity and the loss of specificity resulting from phosphorothioates at higher doses [125]. The mechanism at the basis of the effect on the tumor growth likely relies on the protection of the oligonucleotide provided by the nanocapsules, which may also act as a controlled oligonucleotide release system in the tumor. Thus, the use of phosphorothioates at low doses combined with nanocapsules represented a new and safe therapeutic strategy for antisense therapy. Using the same in vivo experimental model, Toub et al. [56] showed that PIBCA nanocapsules were efficient to deliver siRNAs into tumour (Figure 7). A dramatic inhibition of tumour growth was observed, especially when a higher cumulative dose was employed. In particular, a reduction of the tumour volume to about $80 \%$ was observed allowing a lower daily and cumulative dose and a better efficacy than for encapsulated ASO. For both the doses, naked siRNA had no inhibitory effect on tumour growth.

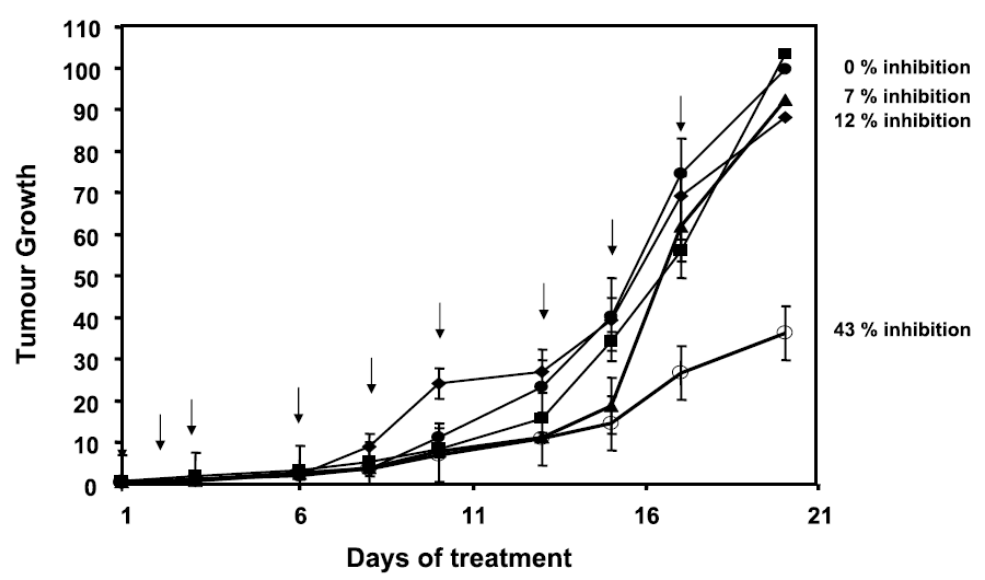

Figure 7. Inhibition of EWS-Fli1-expressing tumor growth in nude mice by siRNA-containing PIBCA nanocapsules at a dose of $0.8 \mathrm{mg} / \mathrm{kg}$. The treatment was performed by intratumoral administration for 20 days. Arrows correspond to the days of treatment. O, AntiSense siRNA-loaded PIBCA nanocapsules; $\boldsymbol{\Lambda}$, control siRNA-loaded PIBCA nanocapsules; $\boldsymbol{\bullet}$, AntiSense siRNA; $\bullet$, control siRNA; $\bullet$, saline. Bars indicate the standard deviation of the mean $(n=6)$. 


\subsection{PACA nanoparticles for peptide and protein delivery}

In the early 90's, several drug delivery scientists have considered the use of nanoparticles for oral or controlled delivery of peptides/proteins. The group of Couvreur has focused much attention over the oral administration of insulin trying to overcome its degradation in the gastrointestinal tract and improve its bioavailability. Interesting results have been obtained with insulin-loaded PIBCA nanocapsules [53]. In diabetic rats, insulin nanocapsules given by a single intragastric administration after an overnight fast, reduced glycemia by 50-60\%. This effect appeared 2 days after administration and was maintained for a period of 20 days. On the contrary, free insulin did not affect glycemia when administered orally under the same experimental conditions [53]. It has been hypothesized that the long-term hypoglycemic effect could be due in part to the progressive arrival of nanocapsules from the stomach to the gut, leading to a delayed absorption. Grislain et al. [58] have found an intense radioactivity signal in the gastrointestinal tract of rats $4 \mathrm{~h}$ after administration of radiolabeled PACA nanospheres. Radioactivity was still present in the mucosa $24 \mathrm{~h}$ later. After arrival into the gut, insulinloaded nanocapsules may be protected against proteolytic enzymes and absorbed by the intestinal mucosa. Then, they were probably transported to the liver by the portal route. Thus, a slow process of redistribution from that organ and/or a slow release of insulin from nanocapsules could have occurred. One transport pathway that was put forward was the uptake by M cells. Indeed, M-cells appeared to be the main site for the uptake of PACA nanoparticles after oral administration allowing NP translocation $[126,127]$.

After subcutaneous administration of PIBCA nanospheres, autoradiographic pictures revealed a progressive reduction of the staining at the injection site, suggesting that nanoparticles were slowly biodegraded [128]. In the same study, PIBCA nanoparticles were found to release growth hormone releasing factor (GRF) in a sustained-release manner. Comparison of the AUC of free GRF and GRF loaded into PIBCA nanoparticles showed that, in addition to the "slow release" process, nanoparticles were able to increase the bioavailability of the peptide. This improvement could be attributed to the partial protection from enzymatic degradation which may result from its encapsulation into nanoparticles while the free GRF is quickly metabolized at the injection site [128].

\section{The squalenoylation technology}

\section{The discovery}

Gemcitabine is an antimetabolite (i.e., nucleoside analogue) which has demonstrated activity against a variety of solid tumors [129]. However, its efficacy is dramatically hampered by the short half-life due to rapid metabolization, the limited intracellular penetration, restricted to the presence of specific membrane transporters and occurrence of resistances [130, 131, 132]. Therefore, design of efficient gemcitabine formulations is still an important medical need. To protect gemcitabine from degradation, a variety of lipid prodrugs has been synthetized by conjugation of acyl chains of different length to the 
amino group in 4' of the drug. However, the covalent modification caused reduction of the aqueous solubility, thus requiring encapsulation in lipid or polymer nanocarriers to allow intravenous administration [133, 134, 135, 136, 137]. Encapsulation of such lipid derivatives in PACA nanoparticles was proposed [138]. However, the drug concentration in the prepared formulations was extremely low reaching a maximum value of only $1 \mathrm{mg} \cdot \mathrm{mL}^{-1}$ for the stearoyl derivative. Expecting that more compact lipid chains would have improved the encapsulation yield, the idea to conjugate gemcitabine to cholesterol was envisioned but was rapidly abandoned due to a possible cardiovascular risks associated to the intravenous administration of cholesterol during a long-term treatment. Thus, attention was shifted to squalene, an acyclic triterpene precursor in the cholesterol biosynthesis. Surprisingly, whatever the initial amount of squalene-gemcitabine (SQGem) conjugate, no free prodrug was detected in the supernatant after centrifugation of PACA nanoparticles, suggesting 100\% drug encapsulation. In front of these results, Patrick Couvreur sensed that squalene-gemcitabine conjugates were able to independently self-assemble into nanoparticles and that the observed $100 \%$ loading eventually corresponded to quantitative formation of SQGem nanoparticles. This assumption was further confirmed without PACA copolymer, where SQGem prodrug self-assembled in water to form nanoparticles without the need of carrier material.

This fortuitous discovery opened the door to an original approach, termed "squalenoylation", that enabled the design of a brand-new family of nanomedicines. It refers to the synthesis of prodrugs resulting from covalent conjugation of the parent drug to a squalene molecule, followed by their selfassembly in aqueous medium into nanoparticles. Considering squalene both acted as a promoiety and drug carrier, this concept led to very high drug loading nanoparticles (much higher than any other traditional drug-loaded nanoparticulate systems) and increased therapeutic efficacy compared to the free drugs. Proposed for the first time in 2006 [139], this versatile concept has been successfully applied to a broad range of different anticancer drugs [140,141, 142, 143, 144], neuroprotective molecules [145], antibiotics [146, 147], antiretrovirals [148, 149], contrast agents [150], ligands [151], small peptides [152, 153, 154], fluorescent dyes,[151] siRNA [155, 156] and also PEG [157]. A complete list of all synthetized prodrugs or conjugates with contrast agents and ligands is reported in Table 1.

Although the idea of synthetizing a prodrug by covalent linkage between a drug and a (macro)molecule [158, 159] was not new, the use of a natural terpenoid such as the squalene as promoiety had never been previously reported. Squalene is abundant in shark oil and largely distributed in human tissues with the highest content found in the skin. In addition, it is well tolerated after intravenous administration or oral injection. Thus, while many of the synthetic materials employed for the formulation of nanomedicines may carry a risk of potential toxicity, immunogenicity or tissue accumulation because of poor degradability/excretion, squalene has a well-known safety profile which made it an ideal biomaterial for the design of drug delivery systems. After the first 
successful results obtained from SQ-based prodrugs, the extreme diversity in chemistry and physical properties of terpenoids prompted further studies on the applicability of the squalenoylation approach to other terpenes for the treatment of severe diseases such as cancer or infections. The groundbreaking nature of this approach led Patrick Coureur being awarded a European Research Council (ERC) Advanced Grant in 2010 (TERNANOMED, FP7-IDEAS-ERC, n²49835). Considering that many natural and biocompatible terpenoids are constituted by isoprene units, the goal of the project was not only to apply the squalenoylation to other drugs and pathologies, but also to identify the influence of the isoprenoid chain length on the capacity (or not) of the resulting prodrug to selfassemble into nanoparticles and to investigate the role of the promoiety on the in vitro/ in vivo pharmacological activity. 
Table 1. List Of Prodrugs Obtained By Covalent Conjugation Of Squalene To Active Molecules, Ligands Or Contrast Agents.

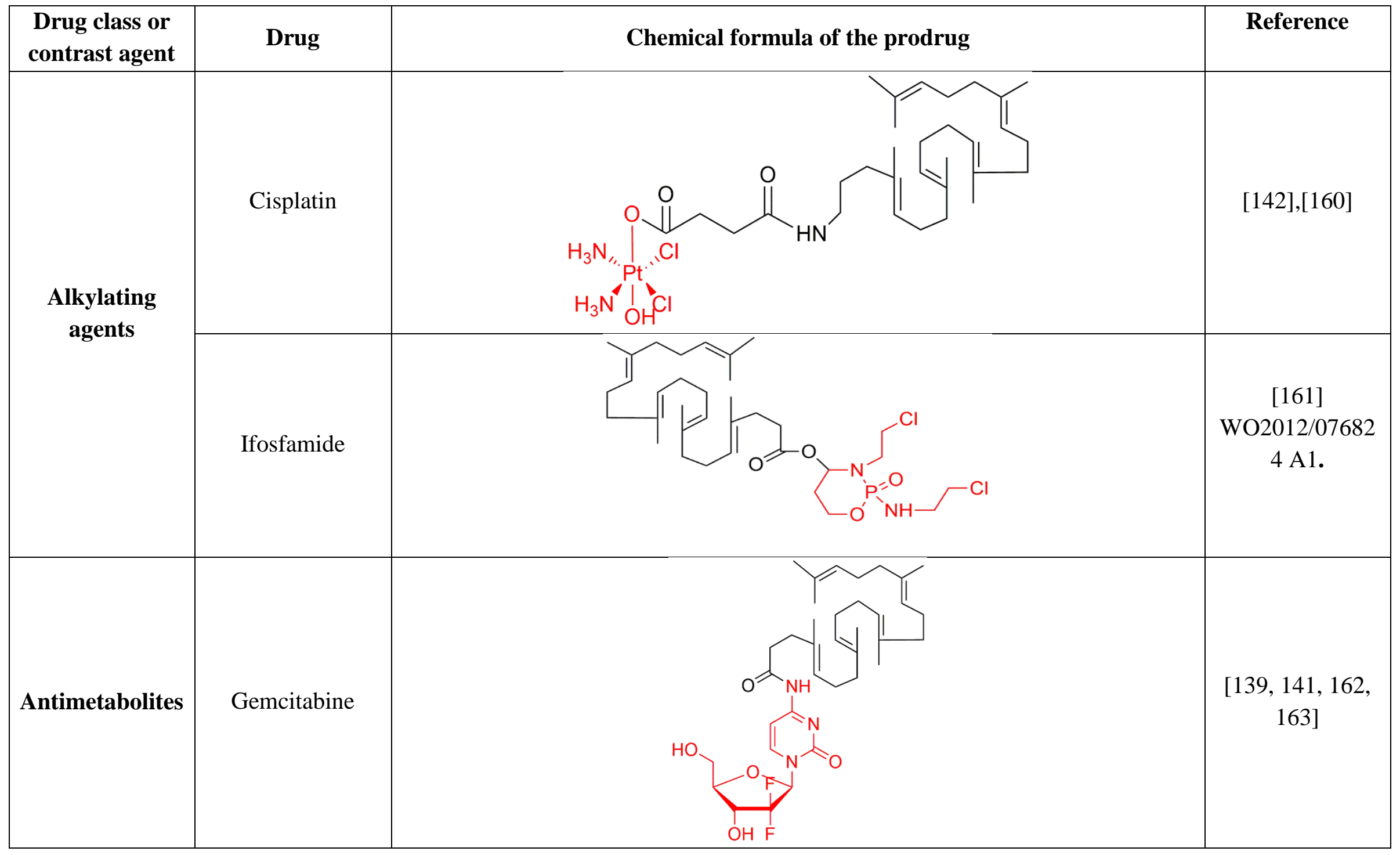




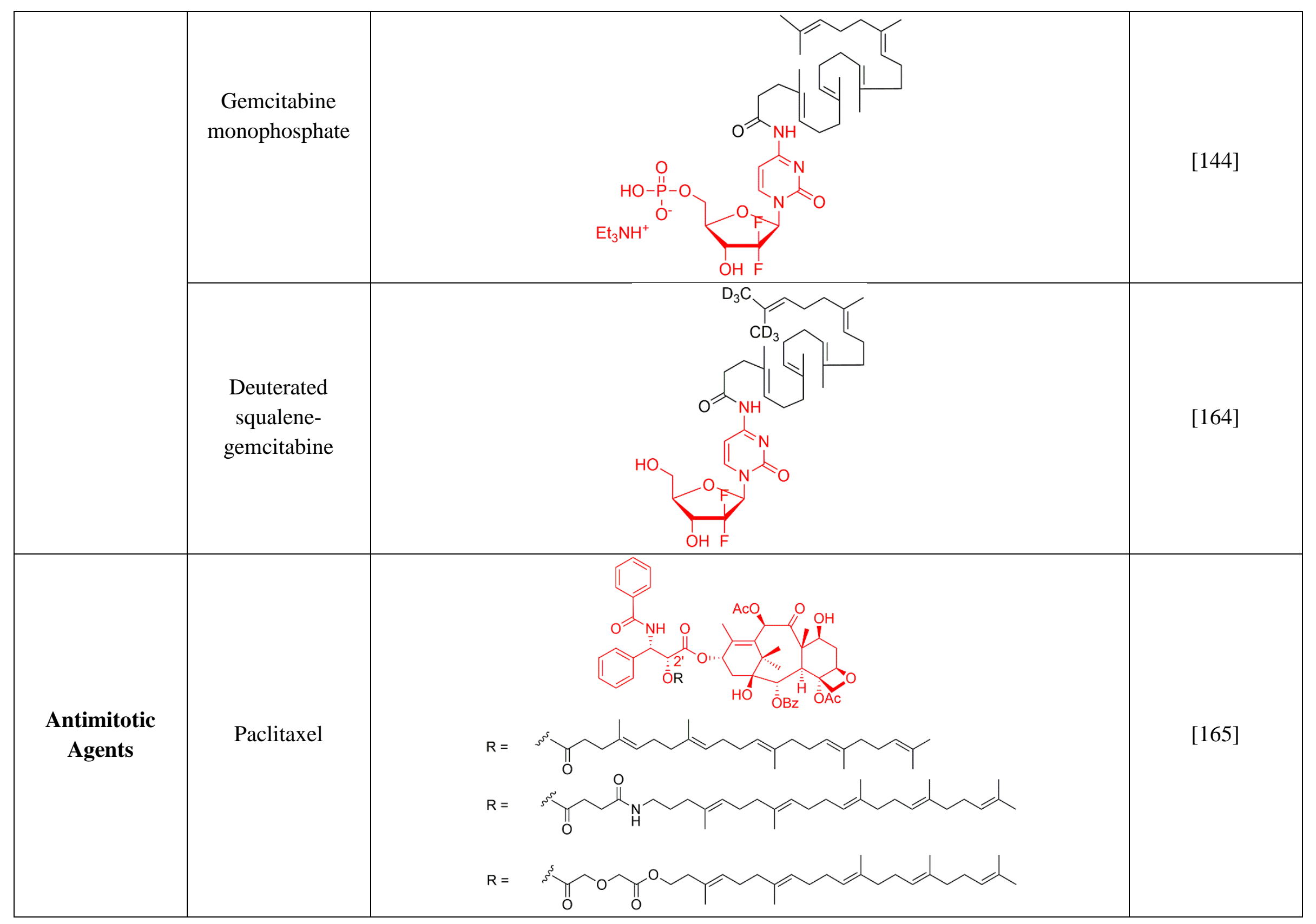




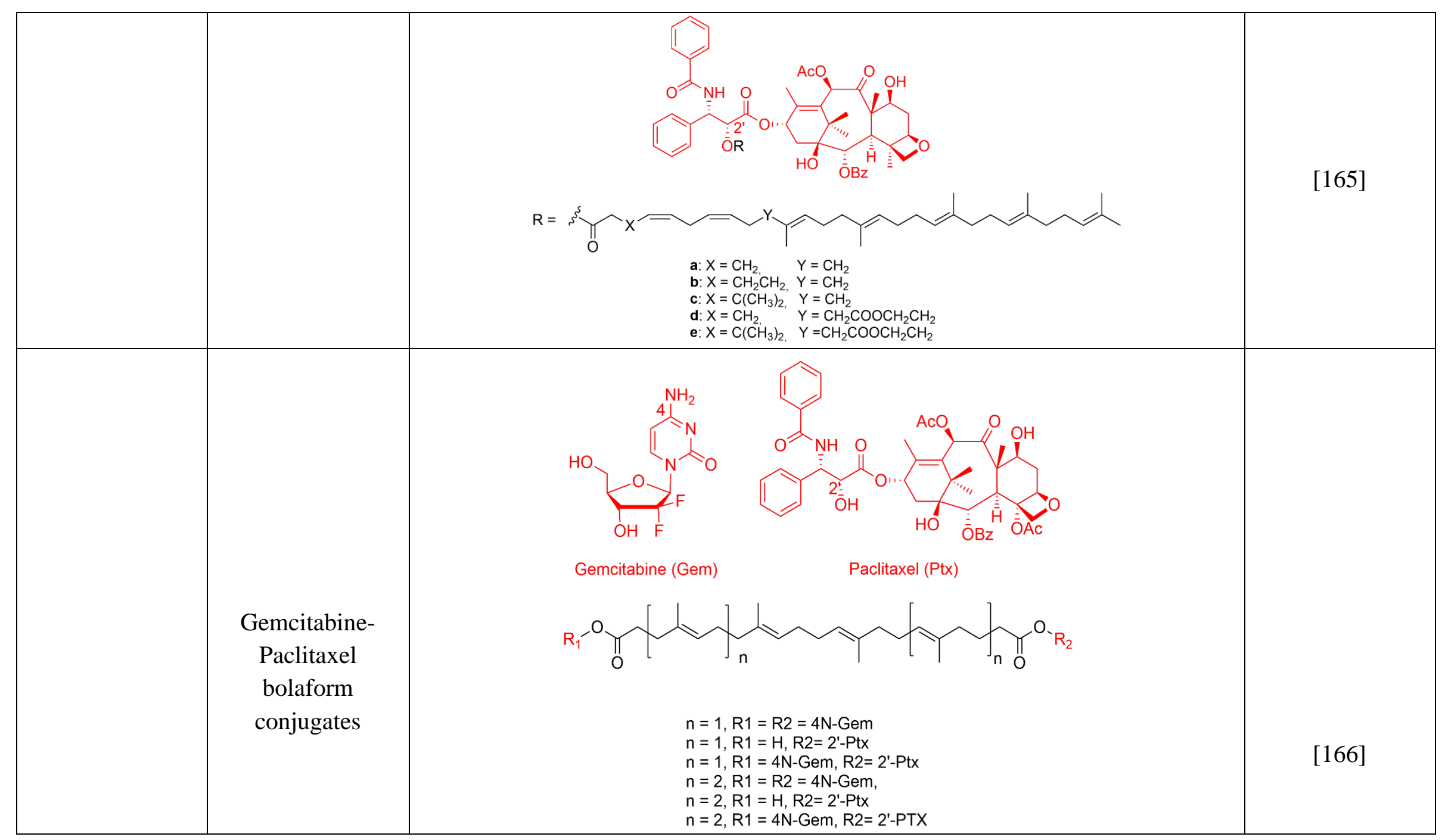




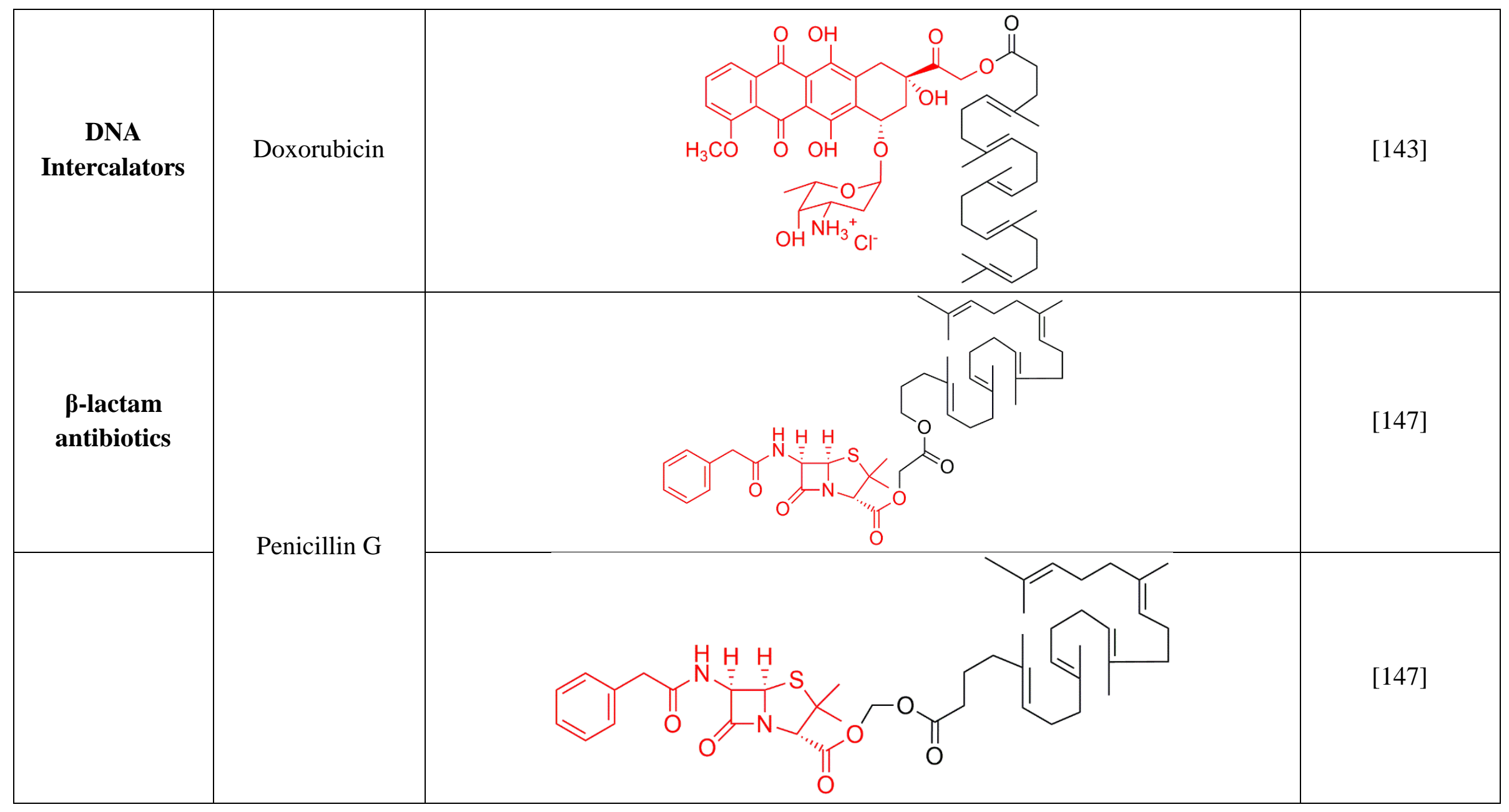




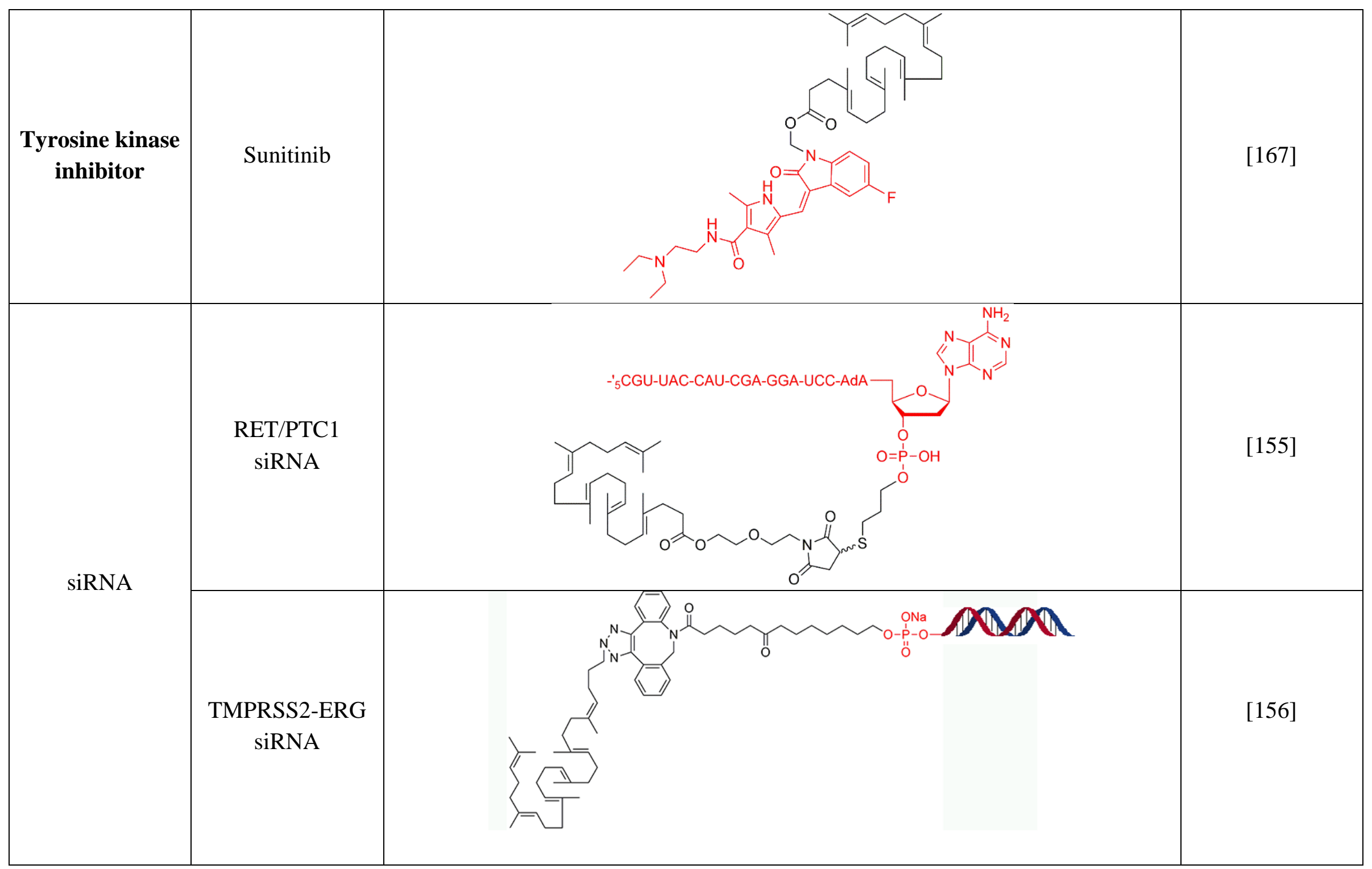




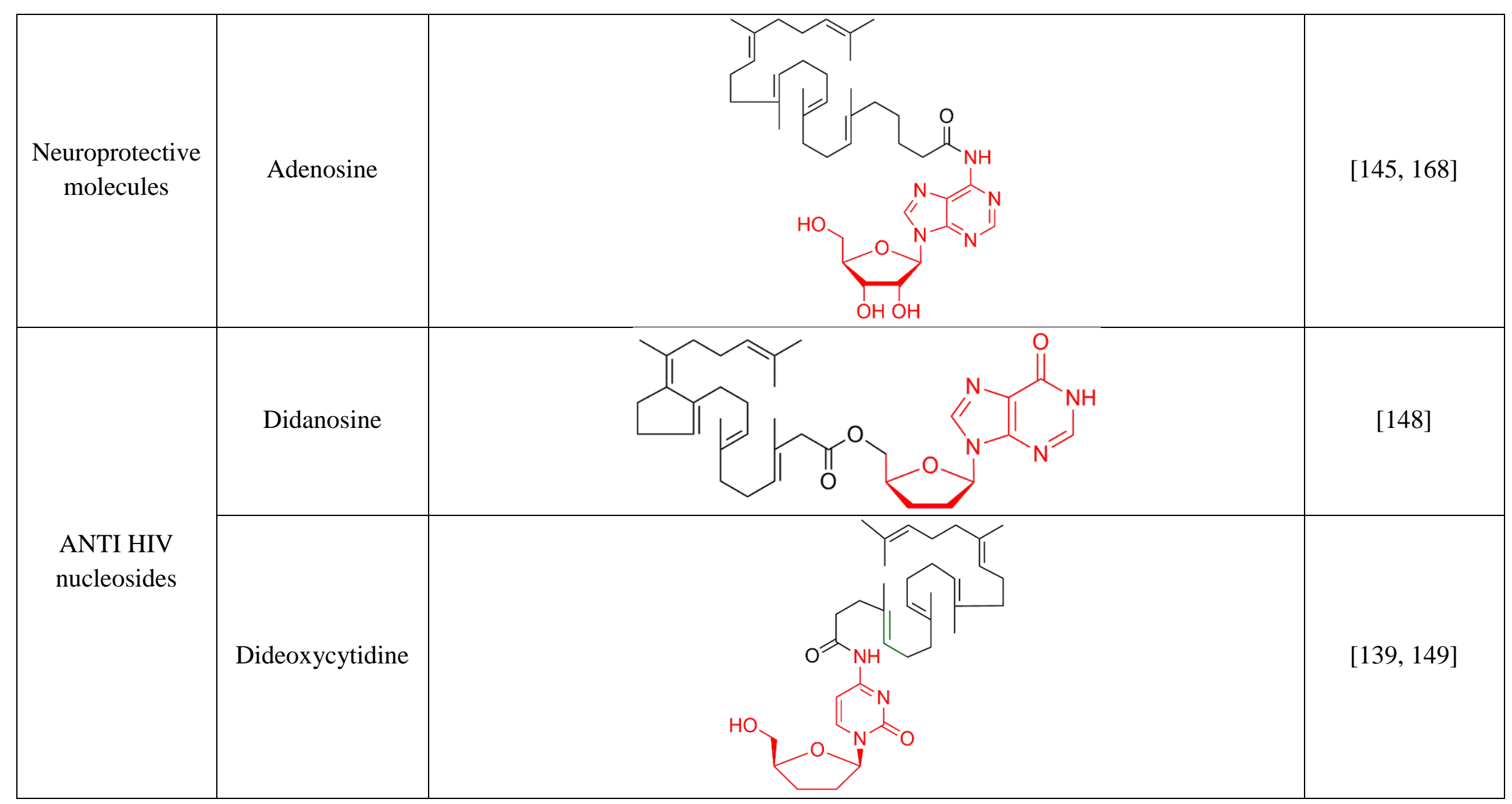




Neuropeptides




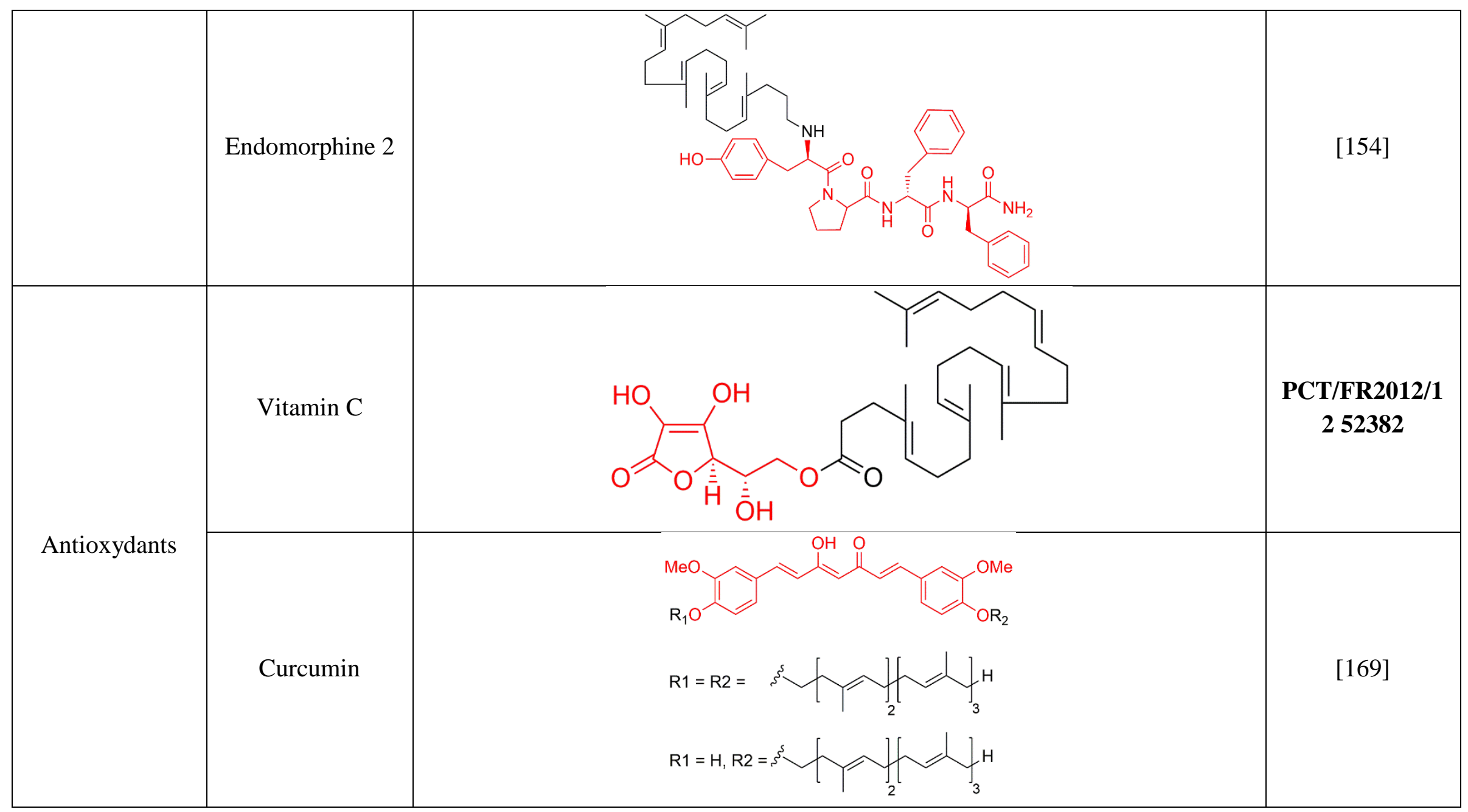




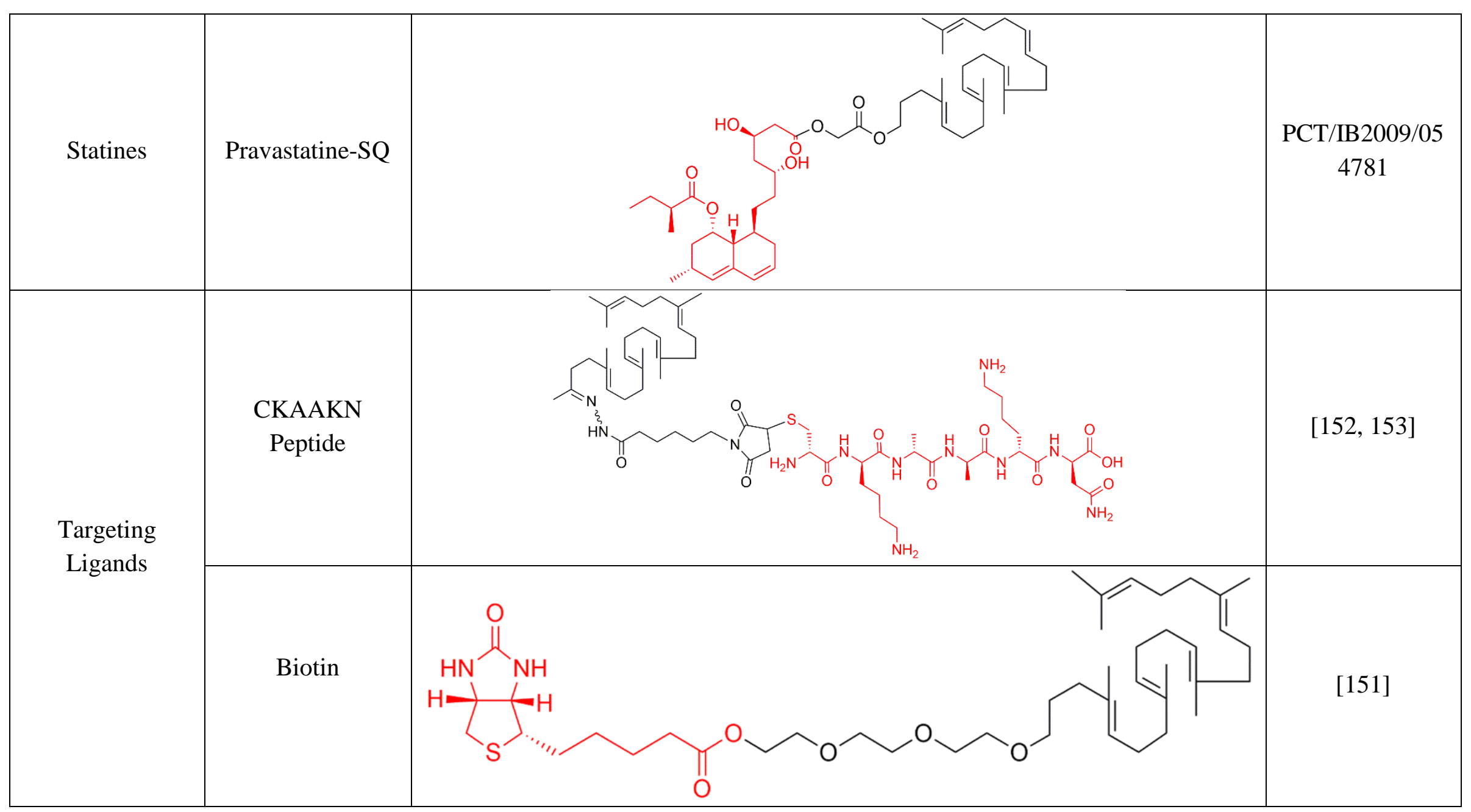




MRI Contrast
agents




\section{General synthetic strategy and formulation}

Squalene is an acyclic triterpene that possesses only non-conjugates, trisubstituted double bonds, without any functional group that would allow for a direct conjugation to drug molecules. Thus, a required preliminary step in the synthesis of all squalene-based prodrugs is the chemical modification of squalene to introduce a functional group at the chain-end suitable for conjugation. This functionalization step was also required for the synthesis of prodrugs in which the SQ moiety has been replaced by polyisoprenoyl chains of different lengths (Figure 8) [170, 171]. Briefly, squalene was readily converted in 2,3-epoxysqualene (1) according to the van Tamelen's method [172], followed by oxidative cleavage to obtain 1,1',2-trisnorsqualene aldehyde (2), a key molecule in the synthetic pathway. Indeed, the aldehyde derivative can then be readily modified to introduce hydroxyl (3), carboxyl (4) or amino groups (5) in the squalene structure to enable its coupling with drugs of interest (Figure 8). The conjugation reaction schema has been opportunely adapted as function of the drug molecule available functional groups.

For instance, the nucleoside analogues derived from cytidine (e.g., gemcitabine, didéoxycytidine) have been coupled to the squalenic acid derivative (4) by simple condensation of the corresponding mixed anhydride, obtained using ethyl chloroformate as an activator, onto the amino group of the nucleoside analogues (Figure 9) [139]. Conjugation to this position allowed for protection of gemcitabine from deamination by deaminases and delayed its metabolization into inactive difluorodeoxyuridine.

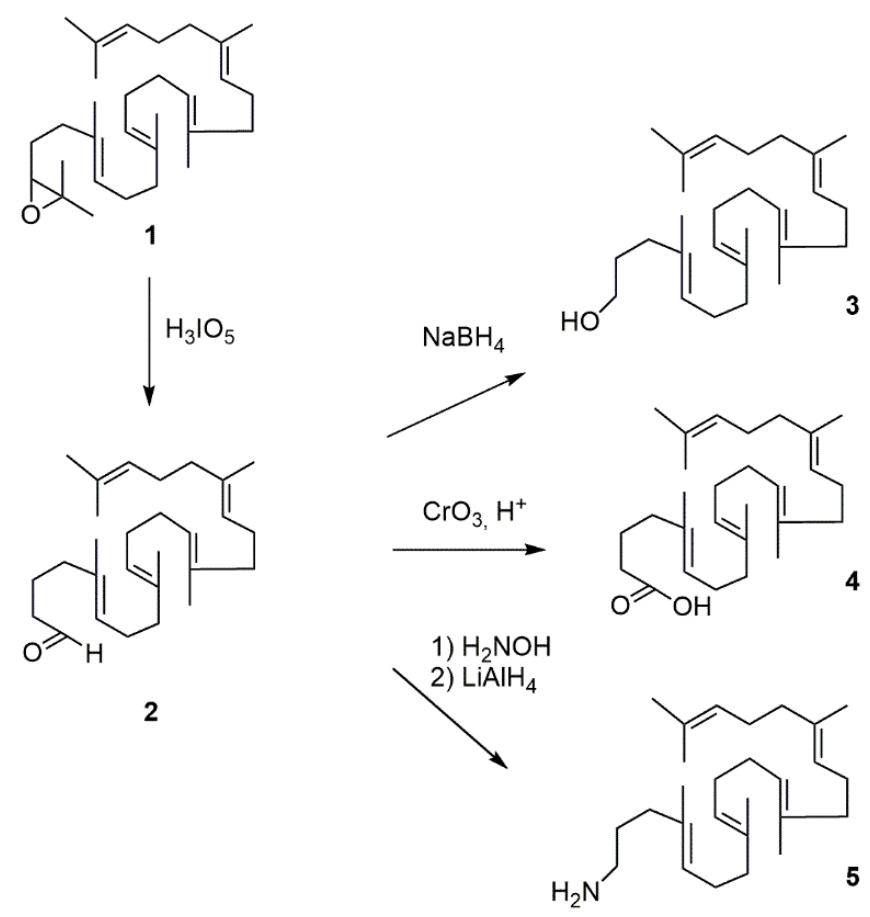

Figure 8. Syntheses of hydroxyl (3), carboxyl (4) and amino (5) squalene derivatives from 2,3epoxysqualene (1) Adapted with permission from ref. [173]. 

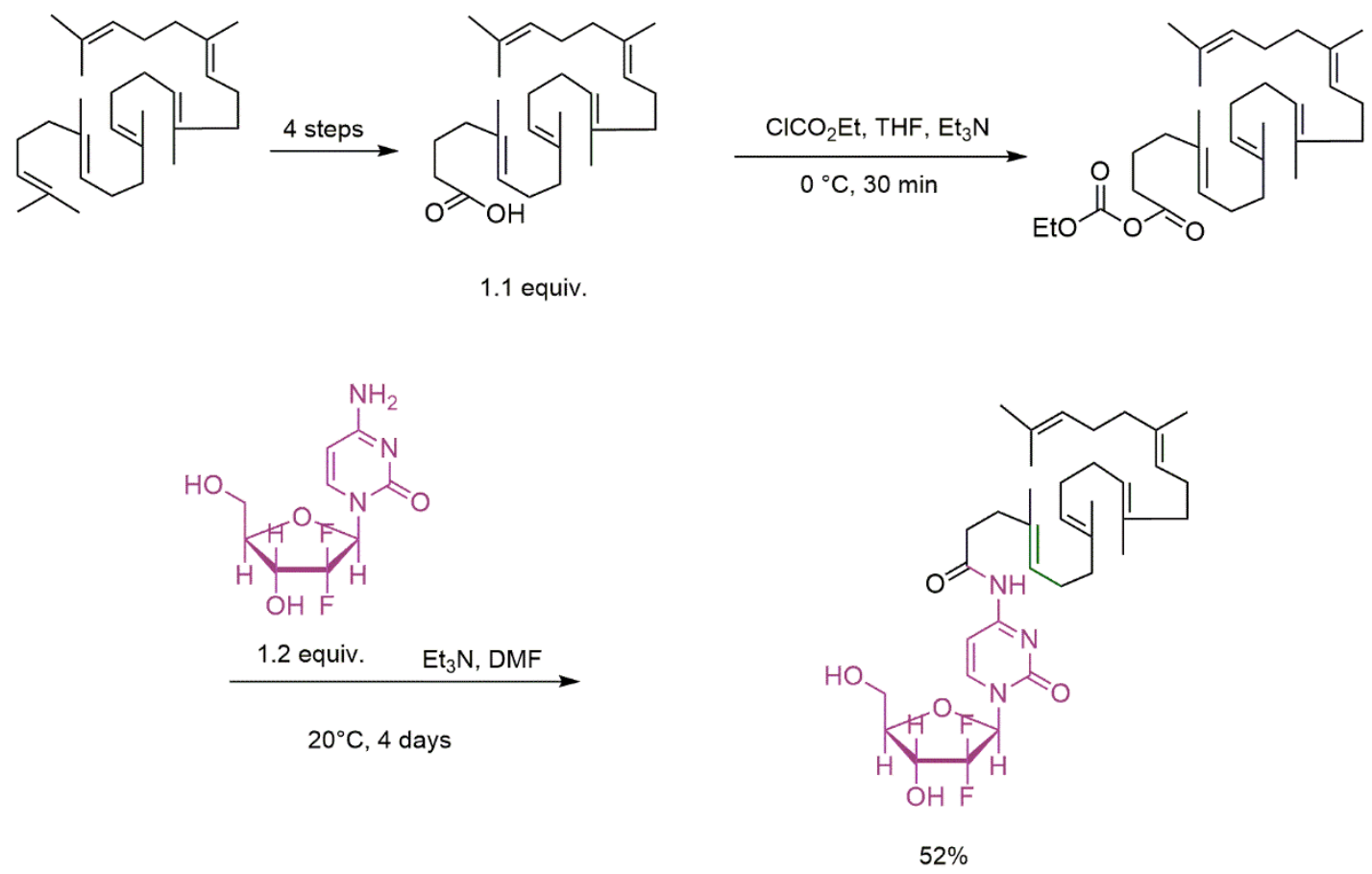

Figure 9. Synthesis of squalene-gemcitabine (SQGem).

For nucleoside analogues lacking the primary amino group on the purine/pyrimidine ring (e.g., didanosine [148]), conjugation was performed by esterification of the $5^{\prime} \mathrm{OH}$ group in the sugar moiety. Similarly, formation of an ester bond allowed for conjugation of squalene to doxorubicin and paclitaxel. For the latter, acylation has been selectively performed on the hydroxyl group in position C2', chosen for its higher accessibility and its crucial role in tubulin polymerization activity [174, 175]. Apart from direct coupling of squalene to paclitaxel, giving an ester linkage, different spacers resulting from intercalation of succinic acid, diglycolic acid, oligo(ethylene glycol) chains as well as dienic units, have been investigated $[165,176]$. By using these more polar/labile and/or less sterically hindered linkers, the idea was to promote release of the drug from the prodrug and therefore the biological activity. Environmentally-sensitive linkers have also been used to conjugate squalene to antibiotics (e.g., penicillin G), allowing spatio-temporal release of the drug and efficient activity against intracellular bacterial infections [147].

Sequential oxidation of cisplatin and condensation with succinic anhydride led to bis-succinyl platinum (IV) complex, that was then linked to squalene amine after ethyl chloroformate activation of the carboxyl groups, to give squalene-cisplatin prodrug [177]. In the case of the adenosine, conjugation of squalenic acid to its amino group did not yield nanoparticles after nanoprecipitation. Stable colloidal nanoparticles were rather obtained when squalenylacetic acid, bearing a longer hydrophobic chain, was used [145]. 
Analogues synthetic pathways were also used to conjugate squalene to macromolecules, such as siRNA [155]. This was achieved by copper-free "click chemistry" through a high yield, reproducible synthesis without by-products [156].

The nanoparticle preparation relied on simple nanoprecipitation of an organic solution of squalene-drug into an aqueous solution (pure water or with $5 \mathrm{wt} . \%$ dextrose) and successive solvent evaporation under reduce pressure. The formulation process does not require the use of any surfaceactive agent, which are generally employed to stabilize most of the nanoscale drug delivery systems, but simply took advantage of the capacity of squalene to adopt a compact conformation in water to form self-stabilized, stable nanoparticles. This feature was likely related to the flexibility of the squalene chain which, during cholesterol biosynthesis, allows interaction with the catalytic pocket of oxydosqualene cyclase enzyme in which the cyclization in lanosterol occurs [178].

The chemical linkage between squalene and the drug enabled $100 \%$ entrapment efficiency without the need for tedious purification steps to remove unloaded drugs. Remarkably, absence of any other materials during formulation resulted in a dramatic increase of drug loading as only squalene molecular weight has to be taken into account (drug loading (wt.\%) $=\mathrm{MW}_{\text {drug }} / \mathrm{MW}_{\text {Sq-drug }}$ ). Extremely high values have been reached while only a few percentages were generally obtained with conventional drug-loaded nanoscale systems. Another advantage, common to any kind of prodrugs, is the absence of "burst release", which is the quick and uncontrolled release of drugs postadministration, and the protection of the drug from early degradation, thus avoiding harmful toxicity until the drug gets cleaved from squalene.

\section{Representative examples of squalene-anticancer drugs}

\subsection{Squalene-gemcitabine}

Among the different squalene-based prodrugs that have been synthesized so far, the most representative and most studied ones is certainly the SQGem $[139,179]$. Nanoprecipitation in water of an ethanolic solution of SQGem resulted in the formation of nanoparticles with average diameter of $130 \mathrm{~nm}$, whose Cryo-TEM images showed a hexagonal/multifaceted shape and an internal structure composed of reticular planes. X-ray diffraction analysis revealed a stacking of direct or inverse cylinders and the packing of SQGem in an inverted hexagonal phase (Figure 10). 

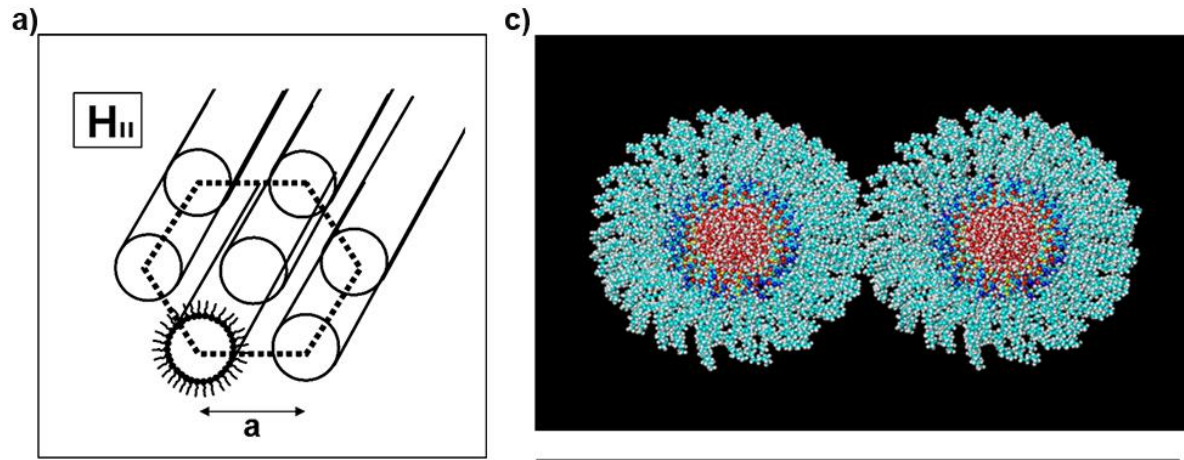

b)
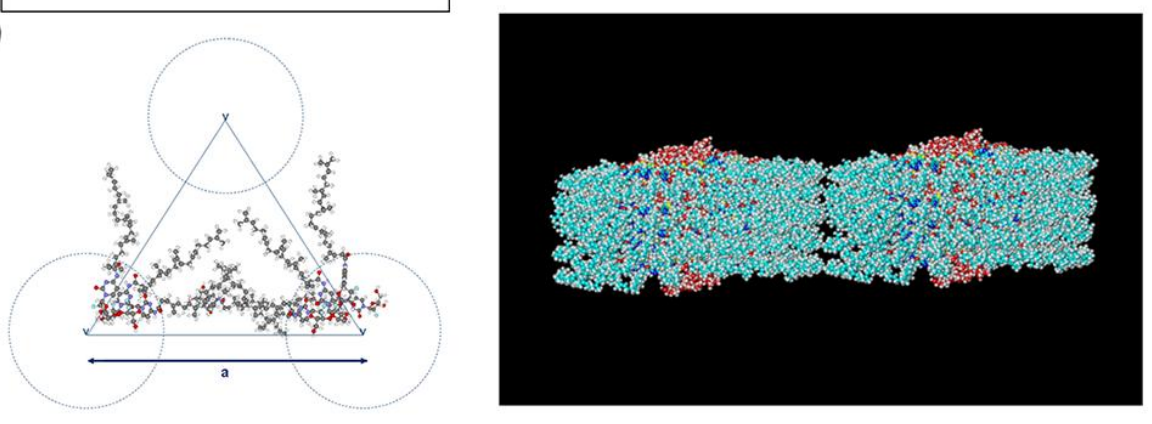

Figure 10. (a) Schematic drawing of the hexagonal $\left(\mathrm{H}_{\mathrm{II}}\right)$ packing of SQGem. (b) SQGem arranged along water channels containing hydrophilic moieties. (c) Face (upper part) and top (lower part) views of a construction of two sections of column, each made of six layers of a disk-like assembly composed of 20 SQGem prodrugs. On the face view, the aqueous core is clearly visible in the center (oxygen/red), and the extent of the polar heterocyclic part can be appreciated by the presence of a circle of pyrimidinone nitrogen atoms (blue) and fluorine atoms (yellow). The top view shows the central inclusion of the water spindle surrounded by lipophilic squalene side chains. Adapted with permission from refs. [173, 179]

Conjugation of squalene to Gem favourably modified the pharmacokinetic, metabolism and biodistribution profiles comparatively to the parent drug. SQGem nanoparticles displayed long circulation with controlled and prolonged drug release, thus increasing the blood $t_{1 / 2}(\sim 3.9$-fold), the mean residence time $(\sim 7.5$-fold) and the distribution volume compared to the drug administered as a free molecule [162]. Consequently, SQGem nanoparticles exhibited improved therapeutic efficacy in various preclinical animal models including subcutaneously grafted tumors and aggressive metastatic leukemia. Moreover, only treatment with SQGem induced a long-term survival both at equimolar and equitoxic doses [140, 141, 163]. These results have been attributed to: (i) improved accumulation of SQGem in the organs of the reticuloendothelial system (i.e., spleen, liver, lung), the major site of metastatic spread; (ii) an intracellular uptake independent from the human equilibrative nucleoside transporters (hENT) [180]; (iii) a slow release of free Gem after cleavage of the prodrug by intracellular cathepsins B and D [139] and (iv) the avoidance of saturation of kinases involved in the phosphorylation of Gem into the active forms responsible of the anticancer activity [181, 182]. 
However, the mechanism by which SQGem nanoparticles accumulated in cancer cells remained unclear for more than a decade until it was recently elucidated [183, 184]. Attention was first focused on evaluation of the interaction between SQGem nanoparticles and the complex microenvironment encountered in the bloodstream. It is composed of proteins, lipoproteins and blood cells, that are known to confer a "biological identity" to nanoparticles [185, 186, 187]. This identity usually governs the nanoparticle fate in vivo, influencing biodistribution, pharmacokinetic, therapeutic efficacy and potential toxicity. The vast majority of studies published so far in this field, relied on identification of adsorbed proteins at the nanocarrier surface, forming the so called "protein corona". However, only limited attention has been devoted to interaction with lipid components of the blood, transported in circulation by lipoproteins (LPs), and with lipoproteins as a whole [188, 189].

The lipidic nature of SQGem nanoparticles and the biosimilarity of squalene with cholesterol, let the Couvreur's group suppose that SQGem had a similar behaviour to that of cholesterol. Thus, SQGem nanoparticles would interact and be transported in the circulation by lipoproteins (LPs), which would act as an indirect endogenous carrier capable to drive SQGem to lipoprotein receptor (LDLR) overexpressing cells such as the cancer ones. Among the different lipoproteins, LDL are the main cholesterol transporters in humans to the peripheral tissues [190] and hence seemed to be the best candidate to perform this indirect targeting. To be noted that the overexpression of LDL receptors in cancer cells and the increased uptake of LDL observed in several tumors [191, 192, 193, 194] reflects their increased metabolic need of cholesterol due to their fast proliferating rate [194, 195, 196].

This hypothesis was confirmed by in vitro and in vivo studies. Incubation with human blood indeed revealed a 500-fold higher affinity between SQGem and LDL compared to albumin (Figure 11a). Analogous behaviour was observed after intravenous administration of SQGem nanoparticles to rats (Figure 11b) and confirmed the tendency of SQGem nanoparticles to accumulate in the main cholesterol transporting particles (that is, LDL in humans and HDL in rodents [197]). Interaction between SQGem nanoparticles and lipoproteins might be imagined as a dynamic process in which the LDL ( $20 \mathrm{~nm}$ in diameter) adsorb at the nanoparticle surface ( 130 $\mathrm{nm}$ in diameter), followed by insertion of SQGem monomers into lipoproteins and further disassembly of SQGem nanoparticles in the biological compartment, as demonstrated by Förster Resonance Energy Transfer (FRET) [184, 198]. 
a)

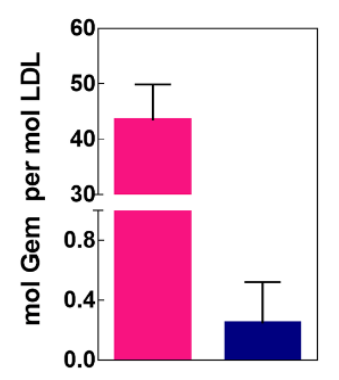

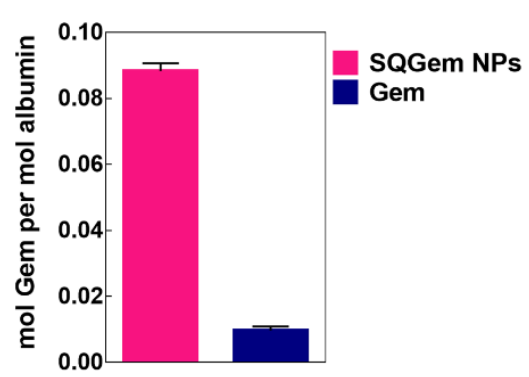

b)

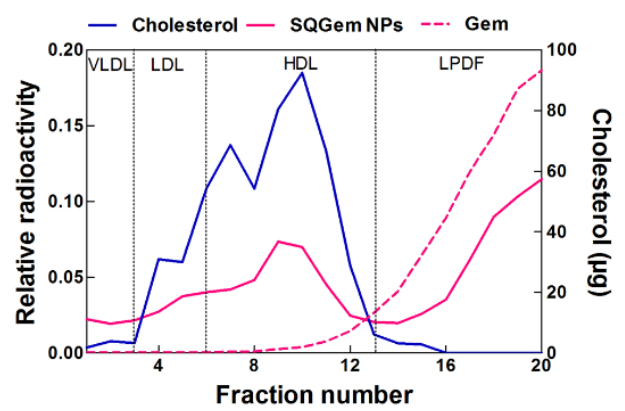

Figure 11. (a) Molar concentration of SQGem and Gem per mole of LDL or albumin after incubation with total human blood (5 min). Bars represent mean \pm standard error of the mean. (b) Radioactivity (magenta lines) and cholesterol (blue line) distribution among the collected fractions of plasma obtained from rats treated with ${ }^{3} \mathrm{H}-\mathrm{SQGem}$ or free ${ }^{3} \mathrm{H}-\mathrm{Gem} 5 \mathrm{~min}$ post administration. Results are expressed as relative radioactivity (mean values) compared to total plasma. Adapted with permission from ref. [184]

These experimental evidences were further supported by molecular dynamic simulations which showed that transfer of SQGem to the lipid core of the lipoproteins was energetically favourable and that SQGem was able to penetrate in the lipoprotein core and then be transported in the circulation. Conversely, a significant energy barrier $\left(\sim 60 \mathrm{~kJ}^{\mathrm{mol}}{ }^{-1}\right)$ not only prevented penetration of free Gem into the LDL core but even any simple interaction with their surface (Figure 12). This corroborated the idea of a specific interaction with lipoproteins mediated by squalene moieties.

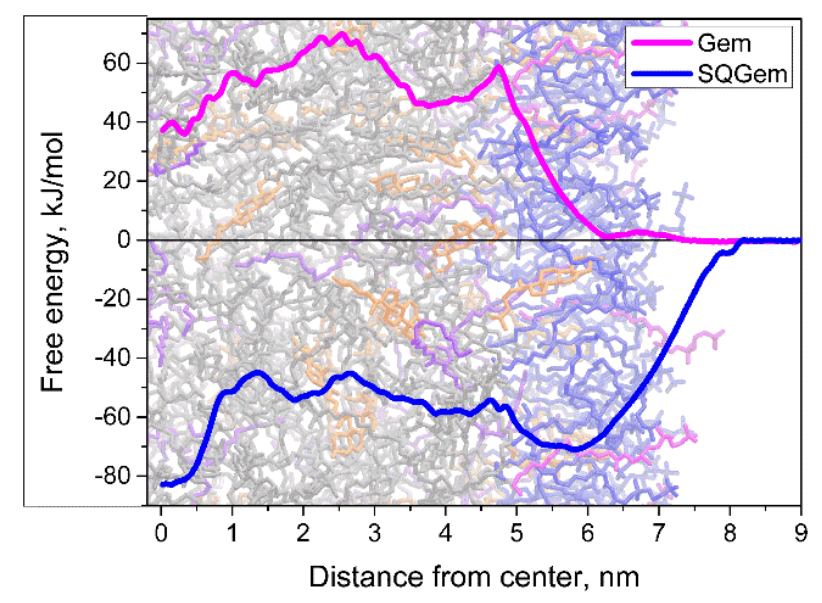

Figure 12. Potentials of mean force of transferring individual Gem (magenta line) and SQGem (blue line) molecules from bulk water to the lipid core of model LDL particle. The plots are superimposed onto a snapshot of the equilibrated LDL system. 1-palmitoyl-2-oleoyl-sn glycero-3-phosphocholine lipids are shown in blue, 1-palmitoyl-2-hydroxy-sn-glycero-3-phosphocholine in red, cholesterol in 
orange, cholesterol oleate in grey and glyceryl trioleate in violet. Adapted with permission from ref. [184]

The biological evaluation demonstrated that lipoproteins were effectively transporting SQGem prodrugs to cancer cells, in which intracellular uptake occurred via LDL receptors. This endogenous transport was also responsible of a higher anticancer activity of SQGem compared to the free drug [183].

Interestingly, such interactions with lipoproteins was not only limited to the SQGem as analogous repartition was also observed for other squalene derivatives [184], thus revealing the key role of the squalene moiety in this drug delivery mechanism. Although the use of the lipoproteins as drug carriers is not a novel concept, the affinity of squalene to lipoproteins has the potential to simplify and enlarge their use as delivery vehicles. Indeed, previously described approaches involved complex isolation of lipoproteins or the synthesis of LDL-like particles in which drugs have to be loaded before administration [199, 200, 201, 202]. Here, thanks to the presence of squalene, it was possible to exploit intravascular events, which spontaneously occurred after intravenous administration of the SQ-based nanoparticles. This biomimetic approach thus represented a novel strategy to deliver anticancer drugs to tumors.

\subsection{Squalene-doxorubicin}

The squalenoylation was also applied to doxorubicin (Doxo) [143]. Nanoprecipitation of SQDoxo led to nanoparticles with a drug loading as high as $57 \mathrm{wt} \%$ and an unexpected loop-train, rod-like morphology as observed by Cryo-TEM, conversely to the vast majority of drug delivery systems that predominantly displayed spherical morphologies (Figure 13).

a)

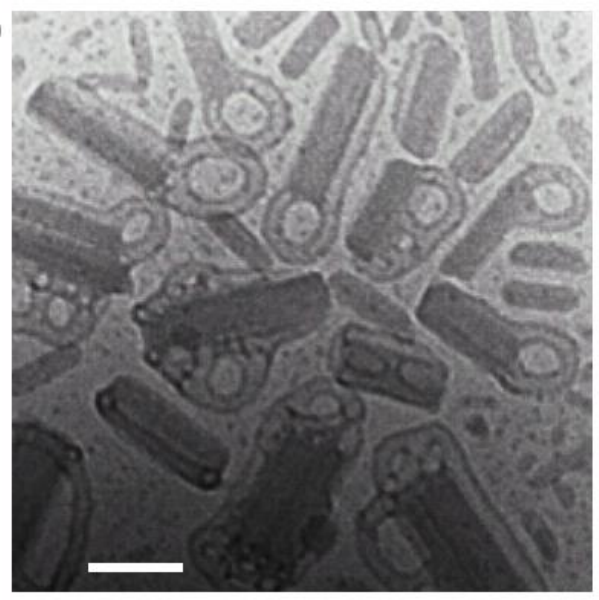

b)

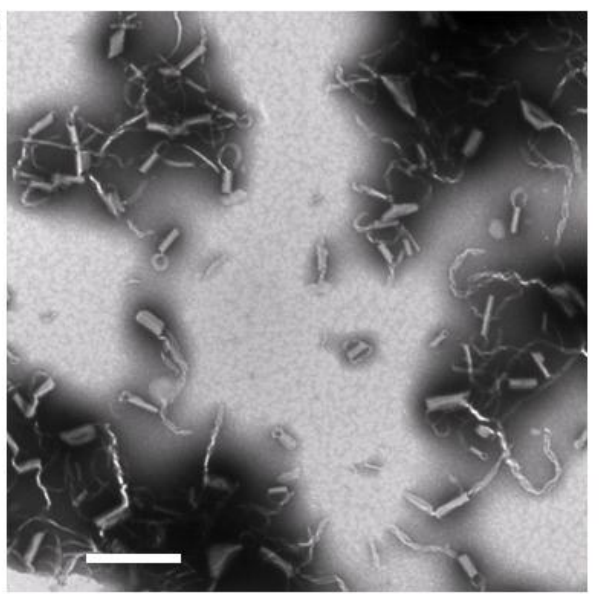

Figure 13. (a) Cryogenic transmission electron microscopy (Cryo-TEM) and (b) transmission electron microscopy (TEM) of SQDoxo nanoparticles. Scale bars: (a) $100 \mathrm{~nm}$ and (b) $500 \mathrm{~nm}$. Adapted with permission from ref. [143]. 
Surprisingly, despite the lack of stealth coating at their surface, SQDoxo nanoparticles significantly prolonged the Doxo circulation time in the bloodstream and reduced its cardiac accumulation compared to the free drug [143]. These results were assigned to the elongated shape of SQDoxo nanoparticles, allowing for their alignment in the bloodstream, in a longitudinal fashion to macrophages, thus preventing their capture. In static conditions (e.g., in vitro in cell culture experiment), however, a strong uptake by cancer cells was observed. These results were in agreement with previous data obtained with filomicelles [203] and clearly highlighted the crucial role of the shape of nanoparticulate systems on their fate following intravenous administration. These so called "nanospaghetti" strongly reduced the cardiac toxicity compared to the free drug, even when tested at 2-fold-higher dose (1 or 2 mg.kg.week equiv. Doxo) in a model of hypertensive rat as attested by absence of myocardial injury either at histological and biochemical (e.g., quantification of troponin-T) level. Moreover, while the free drug negatively affected the food intake causing a significant loss of body weight, no sign of toxicity was observed during the whole study with SQDoxo nanoparticles.

This formulation was even benchmarked against commercial liposomal formulations (i.e., Doxyl, Myocet). In two experimental models of lung (M109) and pancreatic (MiaPACA-2) cancer, SQDoxo nanoparticles led to the highest anticancer efficacy with a tumor mass reduction up to 90 and 95\%, respectively, whereas Myocet and Doxyl were both below 60\% [143]. In agreement with these results, immunohistological evaluation of tumor biopsies revealed the highest apoptotic activity (caspase-3 activation) in the SQDoxo treated group (Figure 14).

a)

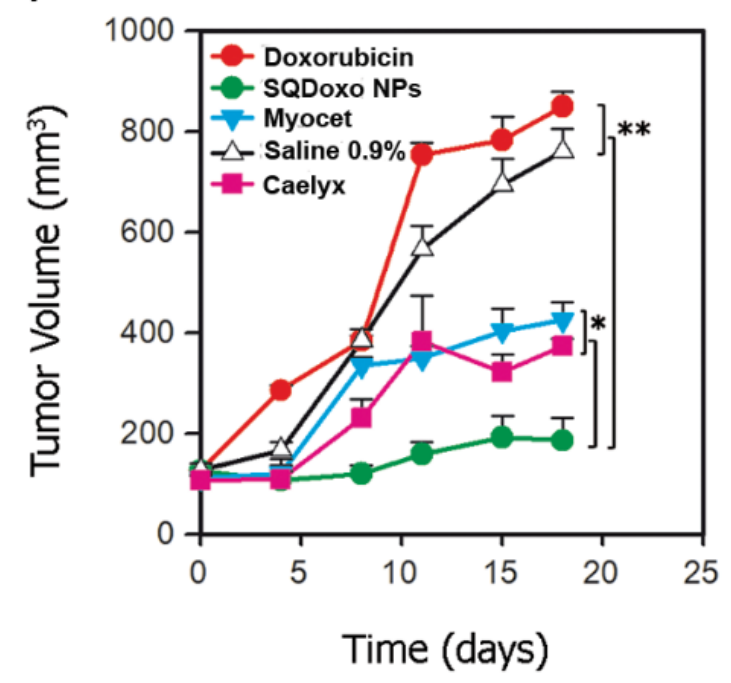

b)

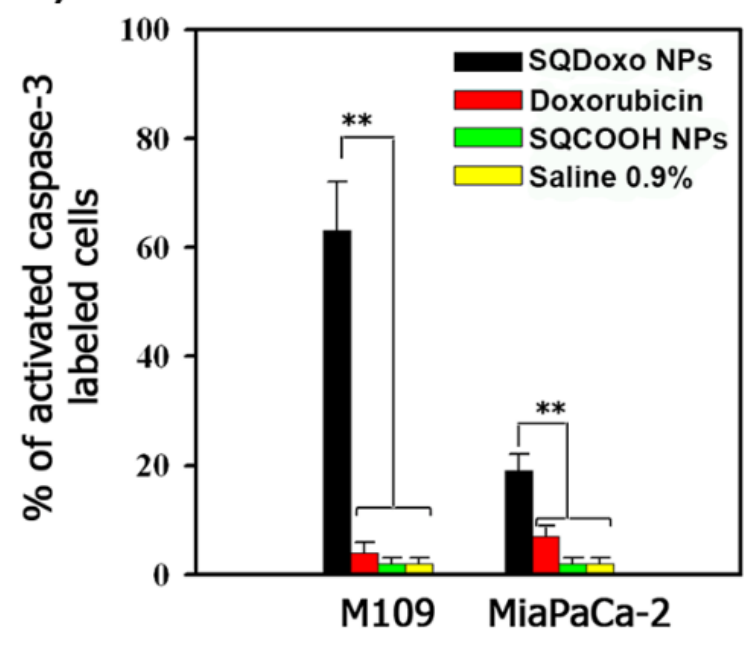

Figure 14. (a) Comparison of the antitumor activity SQDoxo nanoparticles with commercial Doxo liposomal formulations (Myocet and Caelyx) administered to MiaPaCa-2 (human pancreatic) tumorbearing mice $(* \mathrm{p}<0.05$, $* * \mathrm{P}<0.01)$. Results are reported as means $\pm \mathrm{SD}$. (b) Count of activated caspase-3 labelled cells in biopsies of M109 (murine lung) and MiaPaCa-2 tumor collected from mice treated with SQDoxo nanoparticles $(* * p<0.01)$. Adapted with permission from ref. [143]. 


\subsection{Squalene-adenosine}

Disorders of the central nervous systems (CNS) represent a major health problem for which efficient therapeutic strategies are urgently required. However, delivery of drugs to the CNS is extremely challenging. Not only active molecules are rapidly degraded and excreted, but the blood brain barrier (BBB) and the blood-cerebrospinal fluid barrier acts as gatekeepers blocking access to central tissues. These problems are faced for instance by adenosine (Ad) which, despite its therapeutic potential due to its capacity to stimulate neuronal survival, inhibit inflammation and improve the blood flow [204, 205, 206, 207], has never been used for the treatment of CNS disorders because of its shorth half-life and its inability to cross the above-mentioned biological barriers [208, 209, 210]. In this context, by aiming to improve Ad circulation time and promote the BBB crossing by exploiting the affinity of squalene for cell membranes [211], synthesis and biological evaluation of squalene-adenosine (SQAd) nanoparticles were performed [145]. Such NPs showed spherical shape mean diameter of $120 \mathrm{~nm}$ and a unique sponge-like supramolecular organization.

The therapeutic activity of SQAd nanoparticles was evaluated on preclinical animal models of cerebral ischemia and spinal cord injury. In the first model, pre-ischemia intravenous administration of SQAd nanoparticles resulted in a dramatic dose-dependent neuroprotective effect, leading to a reduction of the infarct volume compared to Ad-treated mice and control groups. Such neuroprotective action was also observed when nanoparticles were administered $2 \mathrm{~h}$ post-ischemia, which represented a more clinically relevant condition. As expected, the smaller infarcted area translated to a better functional recovery $24 \mathrm{~h}$ after injury (Figure 15). This protection was likely assigned to an improvement of the cerebral microcirculation consequent to a vasodilatory action, mediated by the released adenosine, on endothelial cells and pericyte/astrocytes receptors. Noteworthy is that despite the multiple receptors on which Ad could act, the treatment did not induce any undesired effect or alteration of haematological, cardiovascular and hepatic biochemical parameters.

The neuroprotective action was also observed in rats which underwent to a T9 contusion, the most relevant model of spinal damage in human [212]. Treatments were administered immediately after the trauma and functional scores of animals were monitored over time for up to 28 days. At $72 \mathrm{~h}$ post trauma, the large damage observed in the cord of rats treated with the free Ad or control formulations strongly affected their locomotory activity and they were unable to move their hindlimbs. Remarkably, no traumatic area was visible in SQAd-treated rats, which were capable to walk without difficulties. These animals reached a score level of 14.4, considered indicative of an axonal activity in the lesioned area, as soon as 9 days post trauma [212]. This result was in good agreement with lower white matter damage and persistence of a higher number of intact small myelinated axons (Figure 16). 

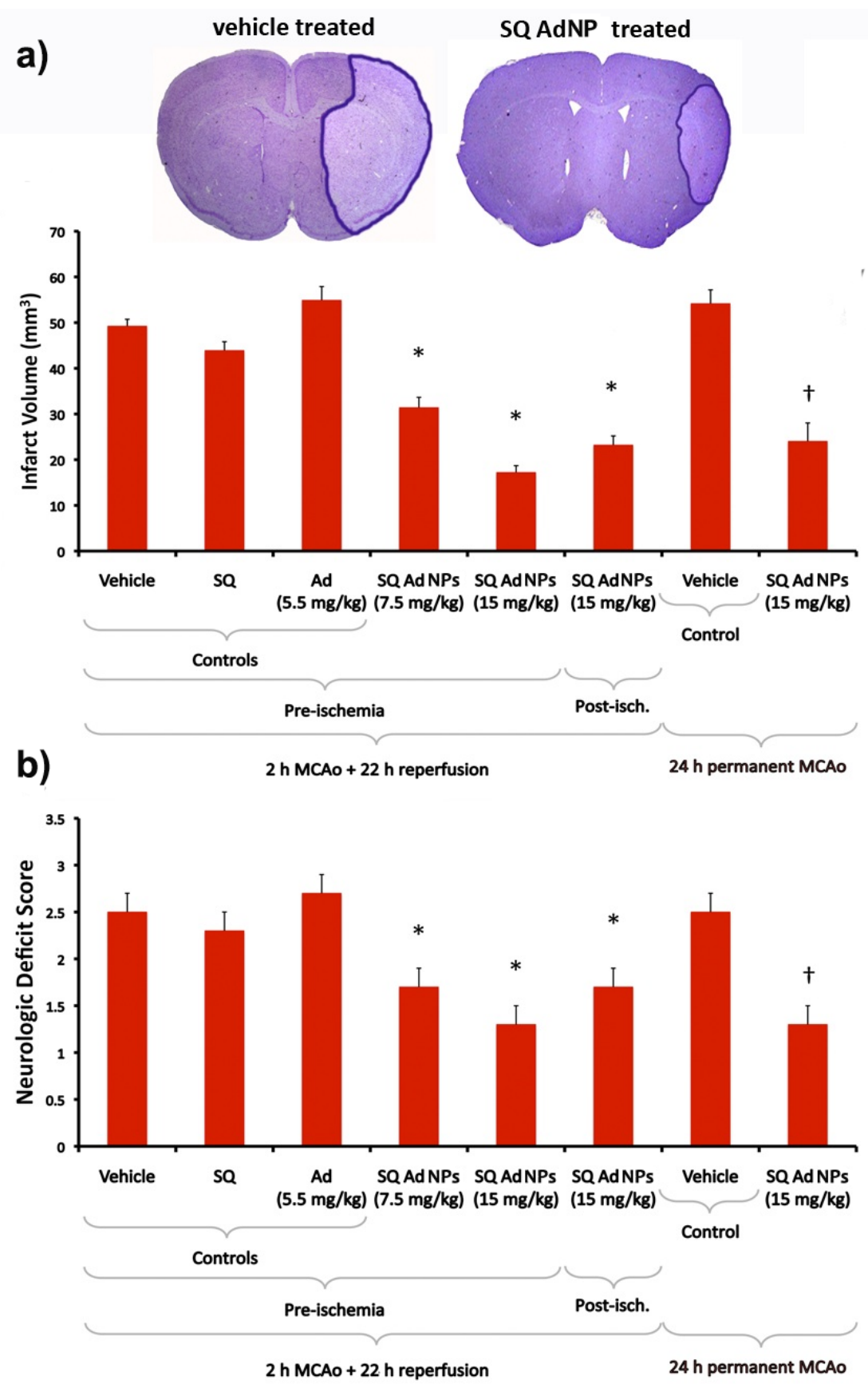

Figure 15. (a) Intravenous administration of SQAd nanoparticles just before or $2 \mathrm{~h}$ post middle cerebral artery occlusion (MCAo) compared to control groups that received vehicle (dextrose 5\%), empty SQ nanoparticles or free Ad. Data presented as mean $\mathrm{mm}^{3} \pm \mathrm{SD} ;{ }^{\dagger}$ and $*$ indicate $\mathrm{p}<0.05$ compared to respective controls. Insets: ischemic volumes in mice subjected to transient $(2 \mathrm{~h} \mathrm{MCAo}$ and $22 \mathrm{~h}$ reperfusion) and permanent ( $24 \mathrm{~h}$ MCAo) ischemia identified by reduced Nissl staining (magnification $\times 10$ ). (b) Neurologic deficit scores assessed $24 \mathrm{~h}$ after the induction of stroke. Data presented as mean $\mathrm{mm}^{3} \pm \mathrm{SD} ;{ }^{\dagger}$ and $*$ indicate $\mathrm{p}<0.05$ compared to respective controls. Adapted with permission from ref. [145]. 


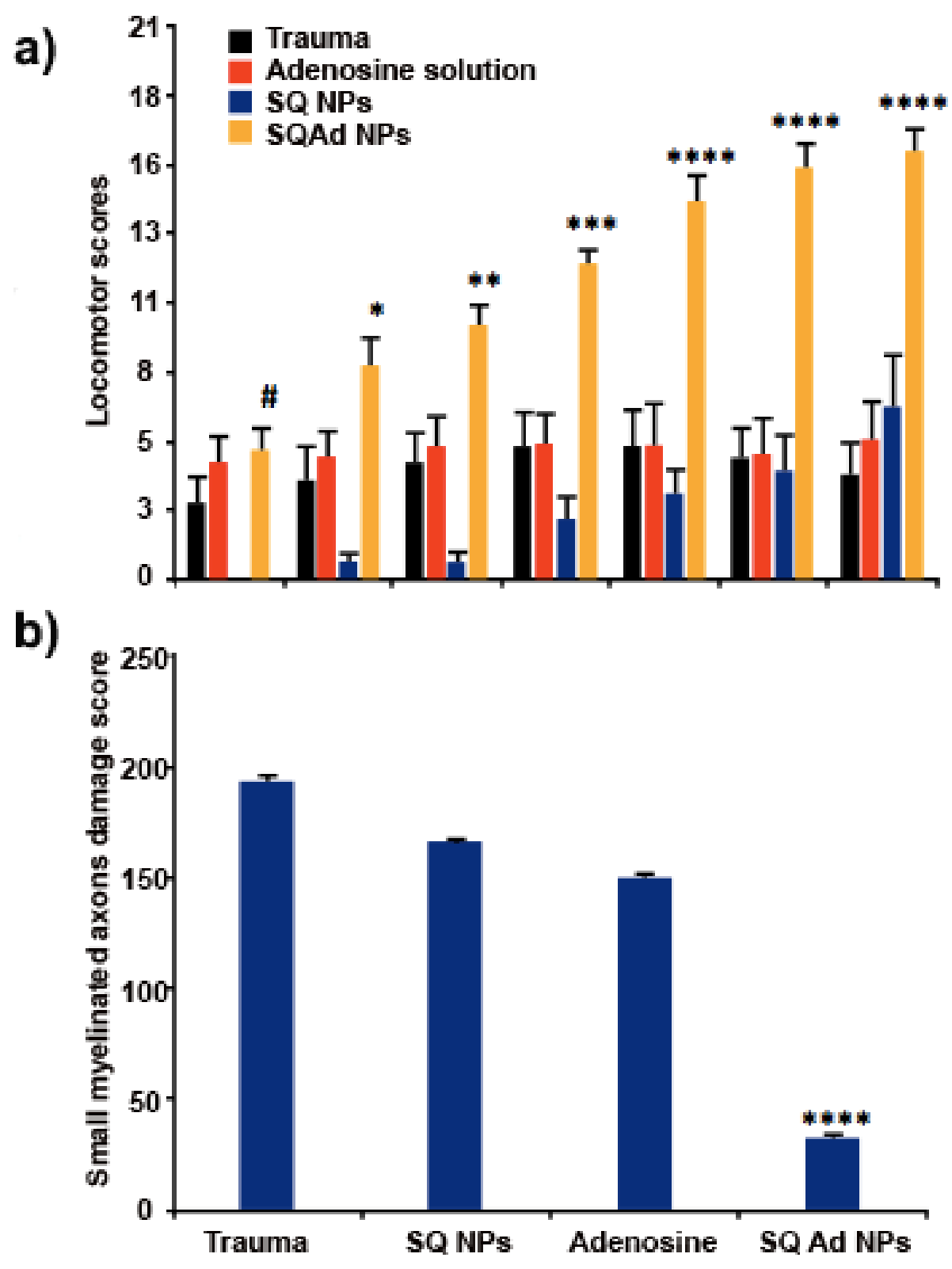

Figure 16. Pharmacological efficiency of SQAd nanoparticles in a model of spinal cord injury in rats. (a) Locomotor scores after 24, 48 and $72 \mathrm{~h}$, and up to 28 days post-trauma using the Basso, Beattie and Bresnahan grading scale. Data presented as mean \pm SEM, \# ns, $* \mathrm{p}<0.05, * * \mathrm{p}<0.01,{ }^{*} * \mathrm{p} p<0.001$, $* * * * \mathrm{p}<0.0001$. (b) Quantification of damages on small myelinated axons. Data are presented as mean $\pm \mathrm{SD}, * * * * \mathrm{p}<0.0001$ compared to all groups. Adapted with permission from ref. [145].

Unexpectedly, no passage of SQAd nanoparticles across the BBB was observed in vitro and their disassembly occurred in the intracellular compartment during translocation as shown by double radioactive labelling and FRET. Conjugation of Ad to squalene protected the drug from early degradation and allowed to form an adenosine reservoir in the circulation, resulting in increased plasma half-life for up to $1 \mathrm{~h}$ post-administration. Conversely, free Ad was rapidly degraded in less 
than 1 min. Consequently, the observed central effect was the result of a direct vascular action mediated by Ad which then induced an indirect neuronal protection. Since it was the first time that such a therapeutic activity had been provided, these results may pave the road to application of squalenoylation in the field of neurosciences.

The versatility of the squalenoylation approach was also illustrated by its application to small interfering RNA for the inhibition of the ret/PTC fusion oncogene in the papillary thyroid carcinoma [155], to antibiotics for the treatment of resistant intracellular infections [146, 147], as well as antiviral molecules for the treatment of HIV-resistant strains [148]. Combination of therapeutic and imaging properties has also been witnessed through construction of a squalene-based nanotheranostic system [177].

\section{Surface modification of squalene-based nanoparticles}

\subsection{PEGylation}

To confer stealth properties to squalene-based nanoparticles, their PEGylation was investigated [157]. It relied on the concomitant self-assembly of a squalene-based prodrug with $2 \mathrm{kDa}$ PEG derivatives; either SQPEG or cholesterol-PEG (CholPEG). This was successfully applied to SQGem and SQ-dideoxycytidine. It was found that, whatever the SQDrug:SQPEG weight ratio (ranging from 1:0.008 to 1:07), SQPEG were efficiently incorporated (72-97 wt.\%) in the resulting composite nanoparticles, as shown by radioactivity studies, zeta potential and size measurements. It was also noted that the higher the PEG derivative amount, the lower the size of the resulting nanoparticles. For instance, SQGem:SQPEG with 1:01; 1:0.3; 1:0.5 and 1:0.7 wt.\% led to 120, 115, 104 and $89 \mathrm{~nm}$, respectively (Figure 17a). It was hypothesized that by anchoring of SQPEG or CholPEG moieties, PEG altered the supramolecular organization of the nanoparticles via swelling of the inverted hexagonal phases. Freeze fracture studies showed that the SQGem nanoparticles prepared using either CholPEG or SQPEG lost their toroidal shape (Figure 17b) to adopt a spherical appearance with a regular inner structure and a golf ball-like shell (Figure 17c). Interestingly, PEG derivatives were also incorporated into already-formed SQGem nanoparticles, leading to slightly lower incorporating efficiencies (71-82 wt.\%) and rapid increase of the average diameter and the particle size distribution. PEGylated SQGem nanoparticles exhibited superior in vitro anticancer activity on Gem-resistant leukemia L1210 10K cells than either their non-PEGylated counterparts (IC50 3 fold higher) or free Gem (IC50 6.4 fold higher) (Figure 17d), likely because of an improved intracellular penetration. Indeed, the relatively smaller size of the SQGem:SQPEG nanoparticles together with the surface activity of SQPEG may induce a temporary perturbation of the cell membrane, facilitating an improved uptake of the nanoparticles. Considering that SQGem nanoparticles are already more efficient than Gem on Gem-resistant L1210 10K cells, presence of SQPEG may still further increase the anticancer activity, thus contributing to resistance reversal. 

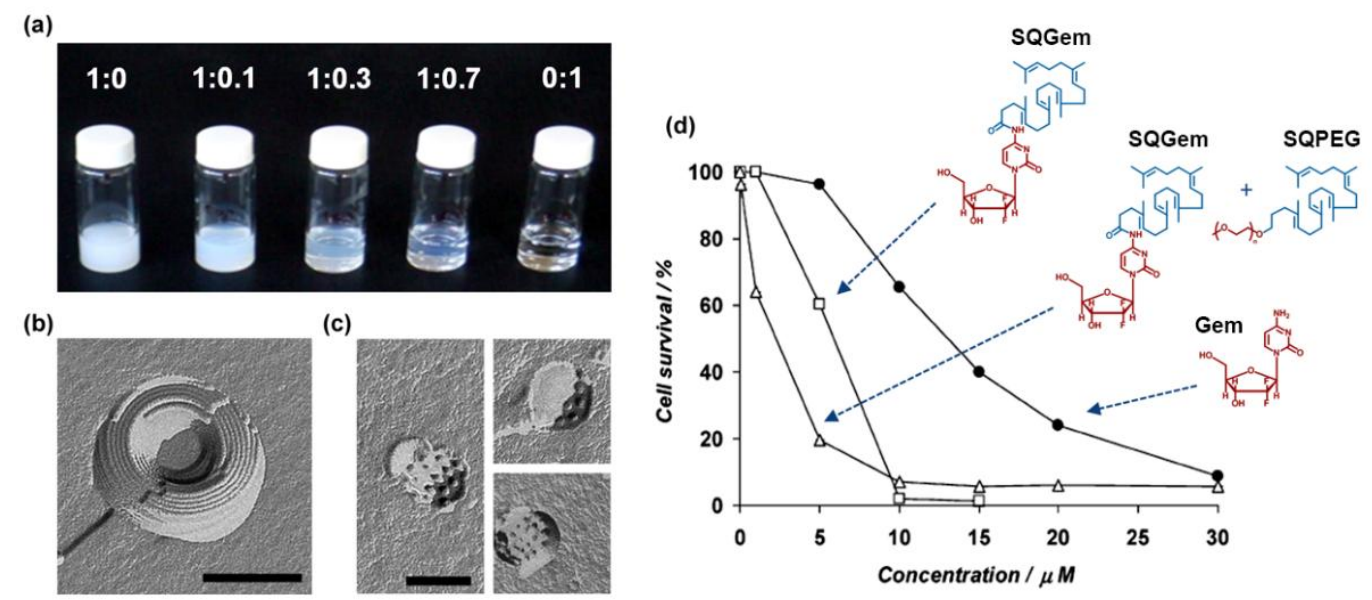

Figure 17. Photographs of the aqueous suspensions of SQGem:SQPEG composite nanoparticles at 50 mg.L -1 $^{-1}$ with various SQGem:SQPEG weight ratios (a). TEM images after freeze-fracture of SQGem (b) and SQGem:SQPEG nanoparticles (c). Scale bars $=100 \mathrm{~nm}$. Cytotoxicity on L1210 10K cells of PEGylated SQGem composite nanoparticles. The values are the mean \pm standard deviation of three determinations. Adapted with permission from ref.[157]

\subsection{Targeted squalene-based nanoparticles}

It was possible to confer both fluorescence and targeting features to SQGem nanoparticles by a simple co-nanoprecipitation approach from the corresponding SQ-based functional components (Figure 18): (i) SQGem; (ii) squalene rhodamine (SQRho) and (iii) squalene-biotin (SQBiotin). Biotin was linked to a triethylene glycol linker to promote its surface exposition and was used as a ligand to selectively target cancer cells via biotin receptors overexpressed at the surface of many cancer cells. The resulting multifunctional nanoparticles were then tested for their tumor targeting ability on MCF7, M109 and HeLa cells by FACS and cytotoxicity assays. Biotin-decorated nanoparticles showed a higher cell internalization and cytotoxicity profile, as opposed to non-biotinylated counterparts. This suggested the surface availability of biotin and the effectiveness of the targeting strategy.

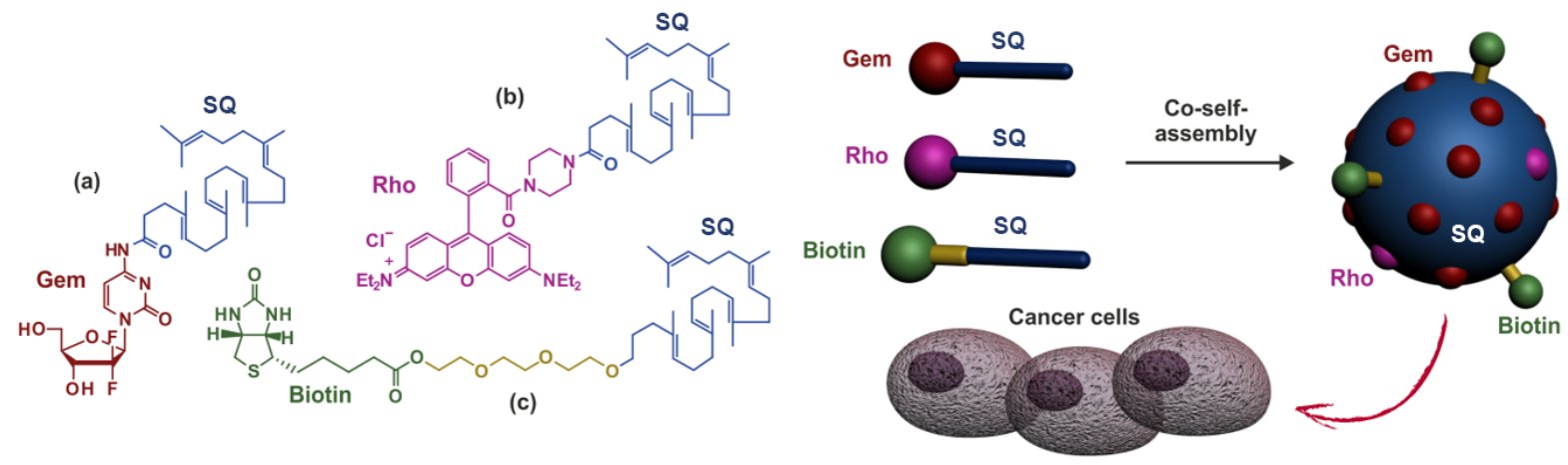

Figure 18. Structure of (a) squalene-gemcitabine (SQGem), (b) squalene-rhodamine (SQRho) and (c) squalene-biotin (SQBiotin), and their co-self-assembly to prepare multifunctional nanoparticles for cancer cell targeting. 
To obtain higher specificity and affinity towards a certain type of cancer, a similar strategy was developed to surface-functionalize SQGem nanoparticles by a six amino acid peptide (CKAAKN) to specifically target pancreatic cancer cells [153]. This peptide was previously identified on RipTag-2 mice (transgenic model of islet cells carcinoma) by in vivo phage display screening [213]. To prepare CKAAKN-functionalized NPs, two approaches have been explored (Figure 19), followed by a systematic investigation to evaluate each strategy in terms of functionalization, colloidal stability and targeting ability in vitro and in vivo $[152,153]$. These approaches relied on: (i) conjugation of CKAAKN to a maleimide squalenoyl derivative (SQMal) leading to formation of the SQCKAAKN and successive co-nanoprecipitation with $\mathrm{SQGem}\left(\mathrm{NPs}_{(\text {before) }}\right.$, Figure 19a) or (ii) co-nanoprecipitation of SQGem and SQMal, and direct conjugation of the CKAAKN peptide at the surface of the NPs $\left(\mathrm{NPs}_{(\text {after })}\right.$, Figure 19b).

Although both synthetic strategies allowed to obtain NPs able to interact with the corresponding receptor, enhanced target binding and higher specific avidity were observed with SQGem_CKAAKN NPs functionalized before NP formation. Therapeutic efficacy of these NPs was evaluated on RipTag2 mice, which spontaneously develop a pancreatic islet cell carcinoma. Interestingly, while both SQGem_CKAAKN NPs and SQGem NPs induced a significant reduction of tumor burden compared to free Gem, the greatest efficacy was observed with the peptide functionalized NPs (tumor reduction by $40 \%$ and by $60 \%$ compared to SQGem NPs and free drug, respectively). In addition, functionalized NPs induced the highest apoptotic rate (increase by $44 \%$ of active caspase-3 immunostaining compared to SQGem NPs). To be noted that functionalized NPs strongly accumulated not only in cancer cell but also in tumor vessels, thus the tumor regression observed with these nanoparticles resulted from both a cytotoxic and an antiangiogenic activity.

a)

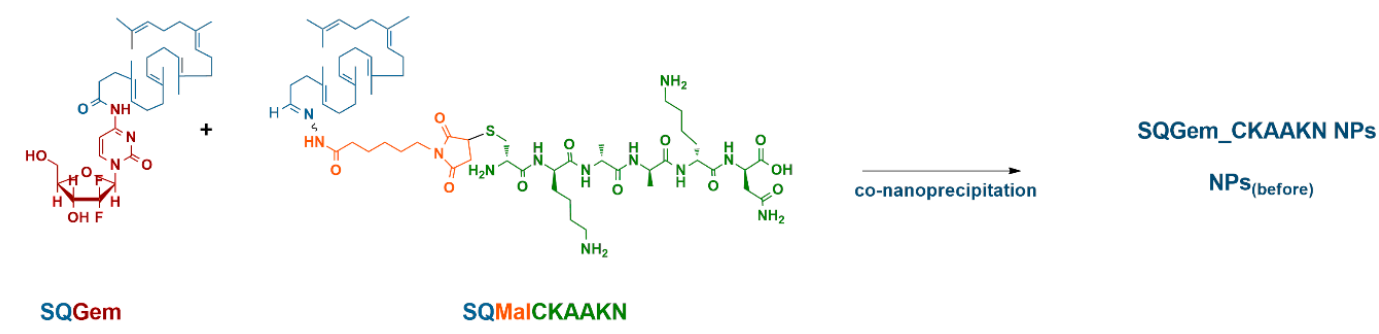

b)

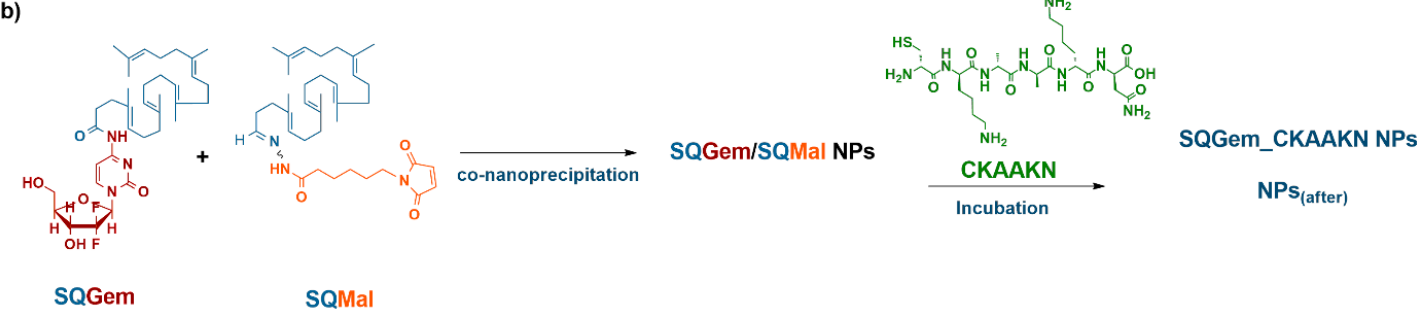


Figure 19. Formulation of CKAAKN-functionalized SQGem nanoparticles: (a) conjugation before NP formation $\left(\mathrm{NPs}_{(\text {before) }}\right)$ and (b) conjugation after $\mathrm{NP}$ formation $\left(\mathrm{NPs}_{(\mathrm{after})}\right)$.

\section{Enlarging the squalenoylation concept}

To investigate the possibility to enlarge the squalenoylation concept to other terpenes, a series of prodrugs was synthetized by conjugation of Gem to polyisoprenoyl chains of variable length (from 1 to 6 isoprene units) (Figure 20) [170]. Prodrugs were obtained by reaction of the corresponding (poly)isoprenic acid with the amino group of Gem, following the reaction scheme already described for SQGem (Figure 9) [139].
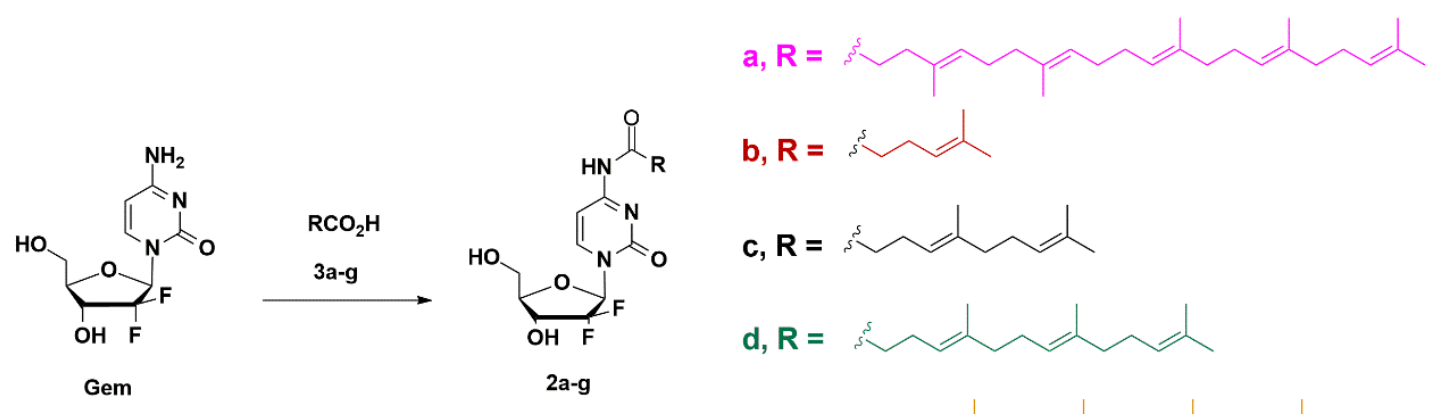

$e, R=$

$f, R=$

$\mathrm{g}, \mathrm{R}=$

Figure 20. Structure of polyisoprenoyl gemcitabine prodrugs (2a-g) obtained by conjugation of the corresponding isoprenic acids (3a-g) to Gem (1). The 2a prodrug corresponds to SQGem. Adapted with permission from ref. [170].

Each prodrug was nanoprecipitated to determine the minimal requisite size of the isoprenoyl chain to allow formation of stable nanoparticles. Only prodrugs containing from 4 to 6 isoprene units led to stable nanoparticles in water while optimization of the formulation conditions was required for shorter polyisoprenoyl prodrugs (e.g., addition of $10 \%$ trehalose or $20 \%(\mathrm{wt} / \mathrm{v})$ of SQPEG) to ensure colloidal stability. Whereas the isoprenoyl chain length dramatically influenced the prodrug ability to form stable nanoparticles, the distribution (symmetrical or not) of the double bonds of the isoprenoyl chain did not seem to affect the self-assembly. In addition, the reduction of the length of the isoprenoyl chain compared to squalene was associated to a faster drug release in serum containing medium, which reached $\sim 30 \%$ in $24 \mathrm{~h}$ for $2 \mathrm{c}$ ( 2 isoprene unit) while only $3 \%$ was released for the SQGem nanoparticles. Further reduction of the chain length down to 1 isoprene unit (2b) did not lead to higher drug release, probably because of the supramolecular organization of the prodrugs, thus reduced enzyme accessibility.

The therapeutic efficacy of $\mathbf{2 d}$ ( 3 isoprene units, the most cytotoxic in vitro) was then evaluated in vivo and compared to SQGem nanoparticles (2a) on an experimental model of pancreatic 
cancer (Mia PaCa-2). While free Gem did not show any efficacy, nanoparticles made of $\mathbf{2 d}$ (3 isoprene units) showed the highest anticancer activity, even higher than SQGem nanoparticles (2a) without inducing any toxicity (Figure 21).

a)

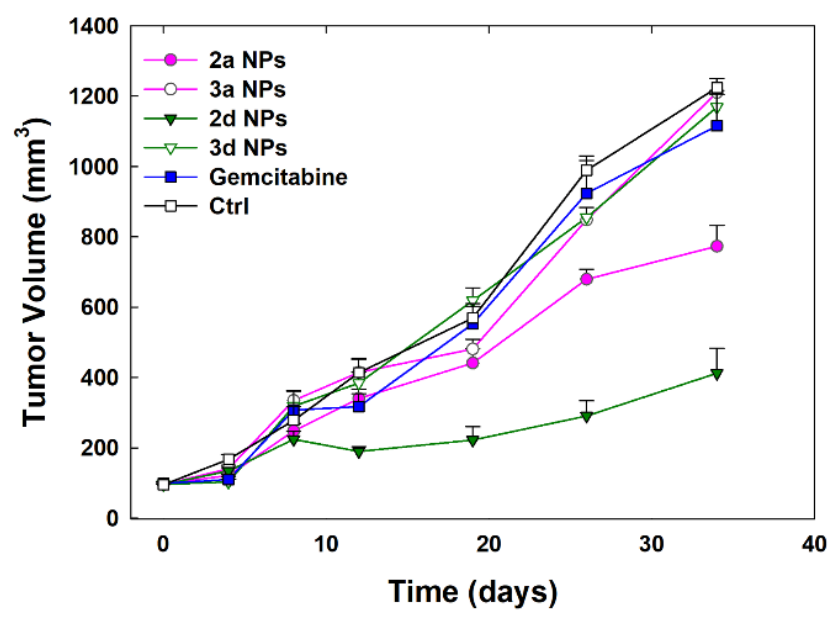

b)

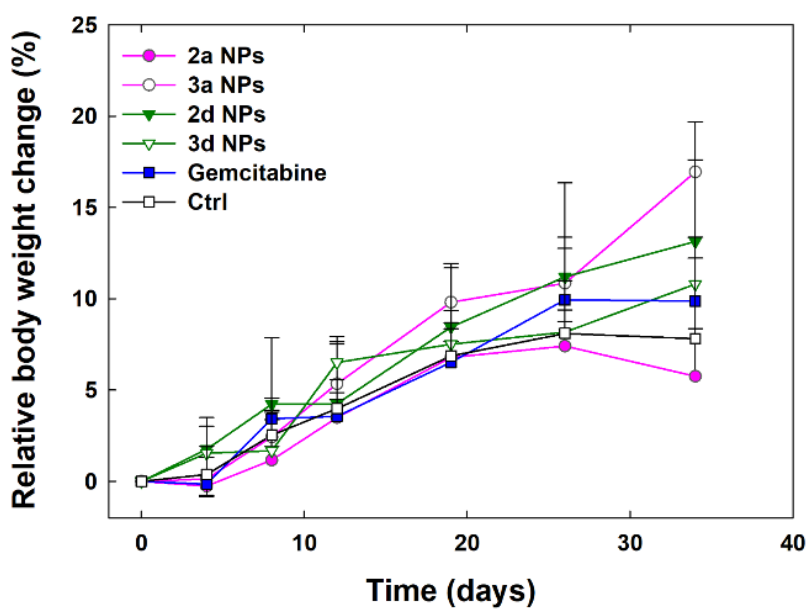

Figure 21. (a) Tumor growth inhibition and (b) relative body weight change in Mia $\mathrm{PaCa}-2$ tumor bearing mice after administration of Gem-based prodrugs with isoprenoyl chain of variable length. Values represent mean \pm SD. Adapted with permission from ref. [170].

Further extension of the squalenoylation concept to other drugs with a different isoprenoyl moiety was illustrated by conjugation of paclitaxel to a single isoprene unit through the hydroxyl group in position C2'of the drug (PtxMIP) [171]. The design of such a prodrug was motivated by the wish to develop an alternative to the Cremophor ${ }^{\circledR}$-containing commercial formulation $\left(\right.$ Taxol $^{\circledR}$ ), whose administration is usually associated to the insurgence of several side effects such as hypersensitivity reactions, hematological toxicity, peripheral neurotoxicity and neuropathy. Nanoprecipitation of an ethanolic solution of PtxMIP into water led to stable NPs conversely to free Ptx that gave formation of typical insoluble needle crystals. Co-nanoprecipitation with SQPEG (8 wt.\%) (PtxMIP_SQPEG NPs) allowed to achieve a concentration suitable for in vivo studies $(4 \mathrm{mg} / \mathrm{mL})$. Noteworthy is that these NPs showed an impressively high drug loading (82 wt.\%), while conventional colloidal formulations of Ptx rarely achieved drug payloads higher than a few percent. This result clearly highlighted the crucial contribution of even a single isoprene unit to the physico-chemical behaviour of the prodrug. Compared to the previously designed SQPtx NPs [165], those formulated from PtxMIP showed significantly higher activity on cancer cells.

Systemic toxicity evaluation revealed a higher tolerability of PtxMIP_SQPEG NPs. Also, no visible tissue irritation could be observed at the injection site. On the contrary, hypersensitivity reaction with clear necrosis of soft tissues, ulceration, oedema and erythema, were observed in the group treated with free Ptx formulation containing Cremophor ${ }^{\circledR}$ (Figure 22). The greater safety profile 
of PtxMIP NPs compared to that of the Ptx allowed for administration of higher doses and resulted in improved anticancer efficacy in vivo on a lung metastatic mouse model of stage IV breast cancer.
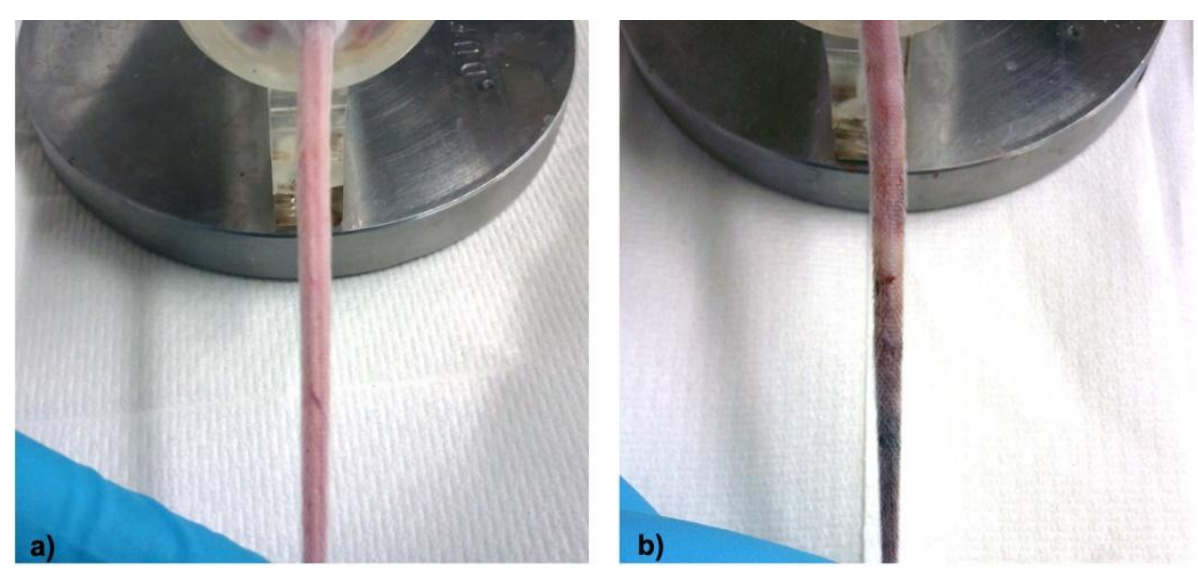

Figure 22. Representative images of a mouse tail after 5 consecutive injections of: (a) PtxMIP_SQPEG NPs and (b) free Ptx at $25 \mathrm{mg} / \mathrm{kg}$. Adapted with permission from ref. [171].

\section{Other routes of administration}

Efficacy of nanoparticles made of squalene prodrugs has been largely demonstrated following their systemic administration via intravenous injection, but promising results have been obtained also when nanoparticles were given orally [142]. Probably thanks to a higher intracellular uptake and the overcome of resistance, the oral treatment with SQcisPt nanoparticles significantly inhibited tumor development on two different model of colon carcinogenesis (i.e., ApcMin/+ mice, and azomethaneinduced) compared to the free drug. The higher efficacy was also associated to a lower toxicity clearly revealing an enhancement of the drug therapeutic index.

\section{Conclusion}

Since the beginning, Patrick Couvreur's scientific career has been marked by important discoveries. Two main axes were considered as major breakthroughs and have had an extraordinary impact on the research work of many young researchers. First, the discovery of biodegradable PACA NPs has shown that it was possible to investigate synthetic materials in a nanoparticulate form at a time when scientists were rather considering using drug carriers produced from natural molecules such as liposomes. The design of PACA NPs led to a Phase III clinical trial with the Livatag $^{\circledR}$ in the treatment of doxorubicin-resistant hepatocarcinoma. Secondly, lessons from the past led to the formulation of NPs from drug-squalene conjugates solving out problems associated to drug encapsulation or leakage. Results from applications of these NPs in the treatment of unmet medical needs (e.g., pancreatic cancer, brain ischemia and spinal cord injury) demonstrated impressive therapeutic effects opening large perspectives for a new generation of novel drug carriers. 


\section{References}

1. Couvreur P, Gillard J, Van den Schrieck HG, et al. Mechanism of disintegration of tablets with a starch base. J Pharm Belg. 1974;29:399-414

2. Couvreur P, Gillard J, Roland M. Influence of water penetration on the disintegration of tablets with a starchy base. Ann Pharm Fr. 1975;33:683-92

3. Couvreur P, Roland M. The desintegration mechanism of pharmaceutical tablets with starch. $\mathrm{J}$ Pharm Belg. 1976;31:511-32

4. Kreuter J. Nanoparticles - a historical perspective. Int J Pharm. 2007;331:1-10

5. Kreuter J, Speiser PP. New adjuvants on a polymethylmethacrylate base. Infect Immun. 1976;13:204-10

6. Couvreur P, Tulkens P, Roland M, et al. Nanocapsules: a new type of lysosomotropic carrier. FEBS Lett. 1977;84:323-6

7. Nicolas J, Couvreur P. Synthesis of poly(alkyl cyanoacrylate)-based colloidal nanomedicines. WIREs Nanomed Nanobiotech. 2009;1:111-127

8. Vauthier C, Dubernet C, Fattal E, et al. Poly(alkylcyanoacrylates) as biodegradable materials for biomedical applications. Adv Drug Deliv Rev. 2003;55:519-48

9. Al Khouri Fallouh N, Roblot-Treupel L, Fessi H, et al. Development of a new process for the manufacture of polyisobutylcyanoacrylate nanocapsules. Int J Pharm. 1986;28:125-132

10. Lambert G, Fattal E, Pinto-Alphandary H, et al. Polyisobutylcyanoacrylate nanocapsules containing an aqueous core as a novel colloidal carrier for the delivery of oligonucleotides. Pharm Res. 2000;17:707-14

11. King ME, Kinney AY. Tissue adhesives: a new method of wound repair. Nurse Pract. 1999;24:66, 69-70, 73-4

12. Oowaki H, Matsuda S, Sakai N, et al. Non-adhesive cyanoacrylate as an embolic material for endovascular neurosurgery. Biomaterials. 2000;21:1039-46

13. Reece TB, Maxey TS, Kron IL. A prospectus on tissue adhesives. Am J Surg. 2001;182:S40S44

14. Hallock GG. Expanded applications for octyl-2-cyanoacrylate as a tissue adhesive. Ann Plast Surg. 2001;46:185-9

15. Marcovich R, Williams AL, Rubin MA, et al. Comparison of 2-octyl cyanoacrylate adhesive, fibrin glue, and suturing for wound closure in the porcine urinary tract. Urology. 2001;57:806810

16. Coover H, Dreifus D, O'Connor J. Cyanoacrylate Adhesives. In: Skeist I, editor. Handbook of Adhesives. New-York: Van Nostrand Reinhold; 1990. p. 463-477.

17. Skeist I, Miron J. In: Bikales N, editor. Encyclopedia of Polymer Science and Engineering. Vol. 2: Wiley-Interscience; 1977. p. 1-19.

18. Pollak JS, White RI, Jr. The use of cyanoacrylate adhesives in peripheral embolization. J Vasc Interv Radiol. 2001;12:907-13

19. Donnelly EF, Johnston DS, Pepper DC, et al. Ionic and zwitterionic polymerization of n-alkyl 2-cyanoacrylates. J Polym Sci Polym Lett Ed. 1977;15:399-405

20. Eromosele IC, Pepper DC, Ryan B. Water effects on the zwitterionic polymerization of cyanoacrylates. Makromol Chem. 1989;190:1613-1622

21. Cronin JP, Pepper DC. Zwitterionic polymerization of butyl cyanoacrylate by triphenylphosphine and pyridine. Makromol Chem. 1988;189:85-102

22. Pepper DC, Ryan B. Initiation processes in polymerizations of alkyl cyanoacrylates by tertiary amines: Inhibition by strong acids. Makromol Chem. 1983;184:383-394

23. Pepper DC. Kinetics and Mechanisms of Zwitterionic Polymerizations of Alkyl Cyanoacrylates. Polym J. 1980;12:629 
24. Johnston DS, Pepper DC. Polymerisation via macrozwitterions, 1. Ethyl and butyl cyanoacrylates polymerised by triethyl and triphenylphosphines. Makromol Chem. 1981;182:393-406

25. Johnston DS, Pepper DC. Polymerisation via macrozwitterions, 3. Ethyl and butyl cyanoacrylates polymerised by benzyldimethyl, triethyl and tribenzylamines. Makromol Chem. 1981;182:421-435

26. Johnston DS, Pepper DC. Polymerisation via macrozwitterions, 2. Ethyl and butyl cyanoacrylates polymerised by pyridine and polyvinylpyridine. Makromol Chem. 1981;182:407-420

27. Eromosele IC, Pepper DC. Anionic polymerization of butyl cyanoacrylate by tetrabutylammonium salts, 1. Initiation processes. Makromol Chem. 1989;190:3085-3094

28. Eromosele IC, Pepper DC. Anionic polymerization of butyl cyanoacrylate by tetrabutylammonium salts, 2. Propagation rate constants. Makromol Chem. 1989;190:30953103

29. Eromosele IC, Pepper DC. Free and paired-ion propagation in the polymerization of butyl cyanoacrylate by tetrabutylammonium salts. Makromol Chem Rapid Commun. 1986;7:639-643

30. Kinsinger JB, Panchak JR, Kelso RL, et al. Methyl $\alpha$-cyanoacrylate. II. Copolymerization studies. J Appl Polym Sci. 1965;9:429-437

31. Yamada B, Yoshioka M, Otsu T. Determination of absolute rate constants for radical polymerization and copolymerization of ethyl $\alpha$-cyanoacrylate in the presence of effective inhibitors against anionic polymerization. Makromol Chem. 1983;184:1025-1033

32. Bevington JC, Jemmett JAL, Onyon PF. Polymerization of methyl $\alpha$-cyanoacrylate-II: Conditions for radical polymerization. Eur Polym J. 1976;12:255-257

33. Otsu T, Yamada B. Determination of Q, e parameters for methyl $\alpha$-cyanoacrylate. Makromol Chem. 1967;110:297-299

34. Canale AJ, Goode WE, Kinsinger JB, et al. Methyl $\alpha$-cyanoacrylate. I. Free-radical homopolymerization. J Appl Polym Sci. 1960;4:231-236

35. Couvreur $\mathrm{P}$, Kante $\mathrm{B}$, Roland $\mathrm{M}$, et al. Polycyanoacrylate nanocapsules as potential lysosomotropic carriers: preparation, morphological and sorptive properties. J Pharm Pharmacol. 1979;31:331-2

36. Couvreur P, Roland M, Speiser P, Biodegradable submicroscopic particles containing a biologically active substance and composition containing them. US-4329332 1982.

37. Lambert G, Fattal E, Pinto-Alphandary H, et al. Polyisobutylcyanoacrylate nanocapsules containing an aqueous core for the delivery of oligonucleotides. Int J Pharm. 2001;214:13-6

38. Fessi H, Puisieux F, Devissaguet JP, et al. Nanocapsule formation by interfacial polymer deposition following solvent displacement. Int J Pharm. 1989;55:R1-R4

39. Legrand P, Lesieur S, Bochot A, et al. Influence of polymer behaviour in organic solution on the production of polylactide nanoparticles by nanoprecipitation. Int J Pharm. 2007;344:33-43

40. Seijo B, Fattal E, Roblot-Treupel L, et al. Design of nanoparticles of less than $50 \mathrm{~nm}$ diameter: preparation, characterization and drug loading. Int J Pharm. 1990;62:1-7

41. Lenaerts V, Raymond P, Juhasz J, et al. New method for the preparation of cyanoacrylic nanoparticles with improved colloidal properties. J Pharm Sci. 1989;78:1051-1052

42. Henry-Michelland S, Alonso MJ, Andremont A, et al. Attachment of antibiotics to nanoparticles: preparation, drug-release and antimicrobial activity in vitro. Int $\mathrm{J}$ Pharm. 1987;35:121-127

43. Verdun C, Couvreur P, Vranckx H, et al. Development of a nanoparticle controlled-release formulation for human use. J Control Release. 1986;3:205-210

44. Manil L, Roblot-Treupel L, Couvreur P. Isobutyl cyanoacrylate nanoparticles as a solid phase for an efficient immunoradiometric assay. Biomaterials. 1986;7:212-6

45. Grangier JL, Puygrenier M, Gautier JC, et al. Nanoparticles as carriers for growth hormone releasing factor. J Control Release. 1991;15:3-13

46. Couvreur P, Puisieux F. Nano- and microparticles for the delivery of polypeptides and proteins. Adv Drug Deliv Rev. 1993;10:141-162

47. Guise V, Drouin JY, Benoit J, et al. Vidarabine-loaded nanoparticles: a physicochemical study. Pharm Res. 1990;7:736-41 
48. Alonso MJ, Losa C, Calvo P, et al. Approaches to improve the association of amikacin sulphate to poly(alkylcyanoacrylate) nanoparticles. Int J Pharm. 1991;68:69-76

49. Lenaerts V, Couvreur P, Christiaens-Leyh D, et al. Degradation of poly (isobutyl cyanoacrylate) nanoparticles. Biomaterials. 1984;5:65-8

50. Couvreur $\mathrm{P}$, Kante $\mathrm{B}$, Roland $\mathrm{M}$, et al. Adsorption of antineoplastic drugs to polyalkylcyanoacrylate nanoparticles and their release in calf serum. $J$ Pharm Sci. 1979;68:1521-4

51. Ammoury N, Fessi H, Devissaguet JP, et al. In vitro release kinetic pattern of indomethacin from poly(D,L-lactide) nanocapsules. J Pharm Sci. 1990;79:763-7

52. Aboubakar M, Puisieux F, Couvreur P, et al. Physico-chemical characterization of insulinloaded poly(isobutylcyanoacrylate) nanocapsules obtained by interfacial polymerization. Int $\mathbf{J}$ Pharm. 1999;183:63-6

53. Damge C, Michel C, Aprahamian M, et al. New approach for oral administration of insulin with polyalkylcyanoacrylate nanocapsules as drug carrier. Diabetes. 1988;37:246-51

54. Lowe PJ, Temple CS. Calcitonin and insulin in isobutylcyanoacrylate nanocapsules: protection against proteases and effect on intestinal absorption in rats. J Pharm Pharmacol. 1994;46:547-52

55. Damge C, Vonderscher J, Marbach P, et al. Poly(alkyl cyanoacrylate) nanocapsules as a delivery system in the rat for octreotide, a long-acting somatostatin analogue. J Pharm Pharmacol. 1997;49:949-54

56. Toub N, Bertrand JR, Tamaddon A, et al. Efficacy of siRNA nanocapsules targeted against the EWS-Fli1 oncogene in Ewing sarcoma. Pharm Res. 2006;23:892-900

57. Hillaireau H, Le Doan T, Besnard M, et al. Encapsulation of antiviral nucleotide analogues azidothymidine-triphosphate and cidofovir in poly(iso-butylcyanoacrylate) nanocapsules. Int $\mathbf{J}$ Pharm. 2006;324:37-42

58. Grislain L, Couvreur P, Lenaerts V, et al. Pharmacokinetics and distribution of a biodegradable drug-carrier. Int J Pharm. 1983;15:335-345

59. Fernandez-Urrusuno R, Fattal E, Rodrigues JM, Jr., et al. Effect of polymeric nanoparticle administration on the clearance activity of the mononuclear phagocyte system in mice. $\mathbf{J}$ Biomed Mater Res. 1996;31:401-8

60. Lenaerts V, Nagelkerke JF, Van Berkel TJ, et al. In vivo uptake of polyisobutyl cyanoacrylate nanoparticles by rat liver Kupffer, endothelial, and parenchymal cells. J Pharm Sci. 1984;73:980-2

61. Guiot P, Couvreur P. Quantitative study of the interaction between polybutylcyanoacrylate nanoparticles and mouse peritoneal macrophages in culture. J Pharm Belg. 1983;38:130-4

62. Pinto-Alphandary $\mathrm{H}$, Balland $\mathrm{O}$, Laurent $\mathrm{M}$, et al. Intracellular visualization of ampicillinloaded nanoparticles in peritoneal macrophages infected in vitro with Salmonella typhimurium. Pharm Res. 1994;11:38-46

63. Lherm C, Müller RH, Puisieux F, et al. Alkylcyanoacrylate drug carriers: II. Cytotoxicity of cyanoacrylate nanoparticles with different alkyl chain length. Int J Pharm. 1992;84:13-22

64. Guise V, Jaffray P, Delattre J, et al. Comparative cell uptake of propidium iodide associated with liposomes or nanoparticles. Cell Mol Biol. 1987;33:397-405

65. Verdun C, Brasseur F, Vranckx H, et al. Tissue distribution of doxorubicin associated with polyisohexylcyanoacrylate nanoparticles. Cancer Chemother Pharmacol. 1990;26:13-8

66. Couvreur P, Kante B, Grislain L, et al. Toxicity of polyalkylcyanoacrylate nanoparticles II: Doxorubicin-loaded nanoparticles. J Pharm Sci. 1982;71:790-2

67. Peracchia MT, Vauthier C, Puisieux F, et al. Development of sterically stabilized poly(isobutyl 2-cyanoacrylate) nanoparticles by chemical coupling of poly(ethylene glycol). J Biomed Mater Res. 1997;34:317-326

68. Peracchia MT, Vauthier C, Passirani C, et al. Complement consumption by poly(ethylene glycol) in different conformations chemically coupled to poly(isobutyl 2-cyanoacrylate) nanoparticles. Life Sci. 1997;61:749-761

69. Bravo-Osuna I, Ponchel G, Vauthier C. Tuning of shell and core characteristics of chitosandecorated acrylic nanoparticles. Eur J Pharm Sci. 2007;30:143-154 
70. Bertholon I, Vauthier C, Labarre D. Complement Activation by Core-Shell Poly(isobutylcyanoacrylate)-Polysaccharide Nanoparticles: Influences of Surface Morphology, Length, and Type of Polysaccharide. Pharm Res. 2006;23:1313-1323

71. Bertholon-Rajot I, Labarre D, Vauthier C. Influence of the initiator system, ceriumpolysaccharide, on the surface properties of poly(isobutylcyanoacrylate) nanoparticles. Polymer. 2005;46:1407-1415

72. Chauvierre C, Labarre D, Couvreur P, et al. Radical Emulsion Polymerization of Alkylcyanoacrylates Initiated by the Redox System Dextran-Cerium(IV) under Acidic Aqueous Conditions. Macromolecules. 2003;36:6018-6027

73. Labarre D, Vauthier C, Chauvierre $\mathrm{C}$, et al. Interactions of blood proteins with poly(isobutylcyanoacrylate) nanoparticles decorated with a polysaccharidic brush. Biomaterials. 2005;26:5075-5084

74. Bertholon I, Hommel H, Labarre D, et al. Properties of Polysaccharides Grafted on Nanoparticles Investigated by EPR. Langmuir. 2006;22:5485-5490

75. Chauvierre C, Leclerc L, Labarre D, et al. Enhancing the tolerance of poly(isobutylcyanoacrylate) nanoparticles with a modular surface design. Int $\mathrm{J}$ Pharm. 2007;338:327-332

76. Peracchia MT, Desmaële D, Couvreur P, et al. Synthesis of a Novel Poly(MePEG cyanoacrylate-co-alkyl cyanoacrylate) Amphiphilic Copolymer for Nanoparticle Technology. Macromolecules. 1997;30:846-851

77. Peracchia MT, Desmaele D, d'Angelo J, et al. Synthesis of a novel poly(MePEG-coalkyl)cyanoacrylate, amphiphilic copolymer for the preparation of PEG-coated nanoparticles. In: Diederichs J, Muller R, editors. Future Strategies for Drug Delivery with Particulate Systems 1998. p. 23-28.

78. Peracchia MT, Vauthier C, Desmaele D, et al. Pegylated nanoparticles from a novel methoxypolyethylene glycol cyanoacrylate hexadecyl cyanoacrylate amphiphilic copolymer. Pharm Res. 1998;15:550-556

79. Peracchia MT, Harnisch S, Pinto-Alphandary H, et al. Visualization of in vitro protein-rejecting properties of PEGylated stealth (R) polycyanoacrylate nanoparticles. Biomaterials. 1999;20:1269-1275

80. Peracchia MT, Fattal E, Desmaele D, et al. Stealth (R) PEGylated polycyanoacrylate nanoparticles for intravenous administration and splenic targeting. J Control Release. 1999;60:121-128

81. Garcia-Garcia E, Gil S, Andrieux K, et al. A relevant in vitro rat model for the evaluation of blood-brain barrier translocation of nanoparticles. Cell Mol Life Sci. 2005;62:1400-8

82. Brigger I, Morizet J, Aubert G, et al. Poly(ethylene glycol)-coated hexadecylcyanoacrylate nanospheres display a combined effect for brain tumor targeting. J Pharmacol Exp Ther. 2002;303:928-36

83. Calvo P, Gouritin B, Villarroya $\mathrm{H}$, et al. Quantification and localization of PEGylated polycyanoacrylate nanoparticles in brain and spinal cord during experimental allergic encephalomyelitis in the rat. Eur J Neurosci. 2002;15:1317-1326

84. Garcia-Garcia E, Andrieux K, Gil S, et al. Colloidal carriers and blood-brain barrier (BBB) translocation: A way to deliver drugs to the brain? Int J Pharm. 2005;298:274-292

85. Brambilla D, Verpillot R, Taverna M, et al. New method based on capillary electrophoresis with laser-induced fluorescence detection (CE-LIF) to monitor interaction between nanoparticles and the amyloid-beta peptide. Anal Chem. 2010;82:10083-9

86. Brambilla D, Souguir H, Nicolas J, et al. Colloidal properties of biodegradable nanoparticles influence interaction with amyloid-beta peptide. J Biotechnol. 2011;156:338-40

87. Brambilla D, Verpillot R, Le Droumaguet B, et al. PEGylated nanoparticles bind to and alter amyloid-beta peptide conformation: toward engineering of functional nanomedicines for Alzheimer's disease. ACS Nano. 2012;6:5897-908

88. Brambilla D, Nicolas J, Le Droumaguet B, et al. Design of fluorescently tagged poly(alkyl cyanoacrylate) nanoparticles for human brain endothelial cell imaging. Chemical communications (Cambridge, England). 2010;46:2602-4 
89. Nicolas J, Brambilla D, Carion O, et al. Quantum dot-loaded PEGylated poly(alkyl cyanoacrylate) nanoparticles for in vitro and in vivo imaging. Soft Matter. 2011;7:6187-6193

90. Stella B, Arpicco S, Peracchia MT, et al. Design of folic acid-conjugated nanoparticles for drug targeting. J Pharm Sci. 2000;89:1452-64

91. Stella B, Marsaud V, Arpicco S, et al. Biological characterization of folic acid-conjugated poly(H2NPEGCA-co-HDCA) nanoparticles in cellular models. J Drug Target. 2007;15:146-53

92. Nicolas J, Mura S, Brambilla D, et al. Design, functionalization strategies and biomedical applications of targeted biodegradable/biocompatible polymer-based nanocarriers for drug delivery. Chem Soc Rev. 2013;42:1147-1235

93. Le Droumaguet B, Nicolas J, Brambilla D, et al. Versatile and efficient targeting using a single nanoparticulate platform: application to cancer and Alzheimer's disease. ACS Nano. 2012;6:5866-79

94. Nicolas J, Bensaid F, Desmaële D, et al. Synthesis of Highly Functionalized Poly(alkyl cyanoacrylate) Nanoparticles by Means of Click Chemistry. Macromolecules. 2008;41:84188428

95. Le Droumaguet B, Souguir H, Brambilla D, et al. Selegiline-functionalized, PEGylated poly(alkyl cyanoacrylate) nanoparticles: Investigation of interaction with amyloid- $\beta$ peptide and surface reorganization. Int J Pharm. 2011;416:453-460

96. Carradori D, Balducci C, Re F, et al. Antibody-functionalized polymer nanoparticle leading to memory recovery in Alzheimer's disease-like transgenic mouse model. Nanomedicine. 2018;14:609-618

97. Fattal E, Youssef M, Couvreur P, et al. Treatment of experimental salmonellosis in mice with ampicillin-bound nanoparticles. Antimicrob Agents Chemother. 1989;33:1540-3

98. Fattal E, Rojas J, Roblot-Treupel L, et al. Ampicillin-loaded liposomes and nanoparticles: comparison of drug loading, drug release and in vitro antimicrobial activity. J Microencap. 1991;8:29-36

99. Youssef M, Fattal E, Alonso MJ, et al. Effectiveness of nanoparticle-bound ampicillin in the treatment of Listeria monocytogenes infection in athymic nude mice. Antimicrob Agents Chemother. 1988;32:1204-7

100. Forestier F, Gerrier P, Chaumard C, et al. Effect of nanoparticle-bound ampicillin on the survival of Listeria monocytogenes in mouse peritoneal macrophages. J Antimicrob Chemother. 1992;30:173-9

101. Balland O, Pinto-Alphandary H, Viron A, et al. Intracellular distribution of ampicillin in murine macrophages infected with Salmonella typhimurium and treated with $(3 \mathrm{H})$ ampicillin-loaded nanoparticles. J Antimicrob Chemother. 1996;37:105-115

102. Buchmeier NA, Heffron F. Inhibition of macrophage phagosome-lysosome fusion by Salmonella typhimurium. Infect Immun. 1991;59:2232-8

103. Brasseur F, Couvreur P, Kante B, et al. Actinomycin D adsorbed on polymethylcyanoacrylate nanoparticles: Increased efficiency against an experimental tumor. Eur J Cancer. 1980;16:14411445

104. Chiannilkulchai N, Driouich Z, Benoit JP, et al. Doxorubicin-loaded nanoparticles: increased efficiency in murine hepatic metastases. Sel Cancer Ther. 1989;5:1-11

105. Cuvier C, Roblot-Treupel L, Millot JM, et al. Doxorubicin-loaded nanospheres bypass tumor cell multidrug resistance. Biochem Pharmacol. 1992;44:509-17

106. Kartner N, Ling V. Multidrug resistance in cancer. Sci Am. 1989;260:44-51

107. Roninson IB. Molecular mechanism of multidrug resistance in tumor cells. Clin Physiol Biochem. 1987;5:140-151

108. Gros P, Croop J, Housman D. Mammalian multidrug resistance gene: complete cDNA sequence indicates strong homology to bacterial transport proteins. Cell. 1986;47:371-80

109. Kubiak C, Couvreur P, Manil L, et al. Increased cytotoxicity of nanoparticle-carried Adriamycin in vitro and potentiation by verapamil and amiodarone. Biomaterials. 1989;10:553-6

110. Bennis S, Chapey C, Couvreur P, et al. Enhanced cytotoxicity of doxorubicin encapsulated in polyisohexylcyanoacrylate nanospheres against multidrug-resistant tumour cells in culture. Eur J Cancer. 1994;30A:89-93 
111. Némati F, Dubernet C, de Verdière AC, et al. Some parameters influencing cytotoxicity of free doxorubicin and doxorubicin-loaded nanoparticles in sensitive and multidrug resistant leucemic murine cells: incubation time, number of nanoparticles per cell. Int J Pharm. 1994;102:55-62

112. Colin de Verdiere A, Dubernet C, Nemati F, et al. Uptake of doxorubicin from loaded nanoparticles in multidrug-resistant leukemic murine cells. Cancer Chemother Pharmacol. 1994;33:504-8

113. Pepin X, Attali L, Domrault C, et al. On the use of ion-pair chromatography to elucidate doxorubicin release mechanism from polyalkylcyanoacrylate nanoparticles at the cellular level. J Chromatogr B Biomed Sci Appl. 1997;702:181-91

114. Barraud L, Merle P, Soma E, et al. Increase of doxorubicin sensitivity by doxorubicin-loading into nanoparticles for hepatocellular carcinoma cells in vitro and in vivo. J Hepatol. 2005;42:736-43

115. Kattan J, Droz JP, Couvreur P, et al. Phase I clinical trial and pharmacokinetic evaluation of doxorubicin carried by polyisohexylcyanoacrylate nanoparticles. Invest New Drugs. 1992;10:191-9

116. Merle P, Camus P, Abergel A, et al. Safety and efficacy of intra-arterial hepatic chemotherapy with doxorubicin-loaded nanoparticles in hepatocellular carcinoma. ESMO Open. 2017;2:e000238

117. Fattal E, Vauthier C, Aynie I, et al. Biodegradable polyalkylcyanoacrylate nanoparticles for the delivery of oligonucleotides. J Control Release. 1998;53:137-43

118. Chavany C, Le Doan T, Couvreur P, et al. Polyalkylcyanoacrylate nanoparticles as polymeric carriers for antisense oligonucleotides. Pharm Res. 1992;9:441-9

119. Chavany C, Saison-Behmoaras T, Le Doan T, et al. Adsorption of oligonucleotides onto polyisohexylcyanoacrylate nanoparticles protects them against nucleases and increases their cellular uptake. Pharm Res. 1994;11:1370-8

120. Nakada Y, Fattal E, Foulquier M, et al. Pharmacokinetics and biodistribution of oligonucleotide adsorbed onto poly(isobutylcyanoacrylate) nanoparticles after intravenous administration in mice. Pharm Res. 1996;13:38-43

121. Schwab G, Chavany C, Duroux I, et al. Antisense oligonucleotides adsorbed to polyalkylcyanoacrylate nanoparticles specifically inhibit mutated Ha-ras-mediated cell proliferation and tumorigenicity in nude mice. Proc Natl Acad Sci USA. 1994;91:10460-4

122. Lambert G, Fattal E, Brehier A, et al. Effect of polyisobutylcyanoacrylate nanoparticles and lipofectin loaded with oligonucleotides on cell viability and PKC alpha neosynthesis in HepG2 cells. Biochimie. 1998;80:969-76

123. Lambert G, Bertrand JR, Fattal E, et al. EWS fli-1 antisense nanocapsules inhibits ewing sarcoma-related tumor in mice. Biochem Biophys Res Commun. 2000;279:401-6

124. Tanaka K, Iwakuma T, Harimaya K, et al. EWS-Fli1 antisense oligodeoxynucleotide inhibits proliferation of human Ewing's sarcoma and primitive neuroectodermal tumor cells. J Clin Invest. 1997;99:239-47

125. Stein CA. Phosphorothioate antisense oligodeoxynucleotides: questions of specificity. Trends Biotechnol. 1996;14:147-9

126. Michel C, Aprahamian M, Defontaine L, et al. The effect of site of administration in the gastrointestinal tract on the absorption of insulin from nanocapsules in diabetic rats. J Pharm Pharmacol. 1991;43:1-5

127. Shakweh M, Ponchel G, Fattal E. Particle uptake by Peyer's patches: a pathway for drug and vaccine delivery. Expert Opin Drug Deliv. 2004;1:141-63

128. Gautier JC, Grangier JL, Barbier A, et al. Biodegradable nanoparticles for subcutaneous administration of growth hormone releasing factor (hGRF). J Control Release. 1992;20:67-77

129. Hertel LW, Boder GB, Kroin JS, et al. Evaluation of the antitumor activity of gemcitabine (2',2'difluoro-2'-deoxycytidine). Cancer Res. 1990;50:4417-22

130. Bergman AM, Pinedo HM, Peters GJ. Determinants of resistance to $2^{\prime}, 2^{\prime}$-difluorodeoxycytidine (gemcitabine). Drug Resist Update. 2002;5:19-33

131. Bouffard DY, Laliberté J, Momparler RL. Kinetic studies on 2',2'-difluorodeoxycytidine (gemcitabine) with purified human deoxycytidine kinase and cytidine deaminase. Biochem Pharmacol. 1993;45:1857-1861 
132. Nordh S, Ansari D, Andersson R. hENT1 expression is predictive of gemcitabine outcome in pancreatic cancer: A systematic review. World J Gastroenterol. 2014;20:8482-8490

133. Castelli F, Sarpietro MG, Ceruti M, et al. Characterization of lipophilic gemcitabine prodrugliposomal membrane interaction by differential scanning calorimetry. Mol Pharm. 2006;3:73744

134. Castelli F, Sarpietro MG, Rocco F, et al. Interaction of lipophilic gemcitabine prodrugs with biomembrane models studied by Langmuir-Blodgett technique. J Colloid Interface Sci. 2007;313:363-8

135. Immordino ML, Brusa P, Rocco F, et al. Preparation, characterization, cytotoxicity and pharmacokinetics of liposomes containing lipophilic gemcitabine prodrugs. J Control Release. 2004;100:331-46

136. Brusa P, Immordino ML, Rocco F, et al. Antitumor activity and pharmacokinetics of liposomes containing lipophilic gemcitabine prodrugs. Anticancer Res. 2007;27:195-9

137. Chung WG, Sandoval MA, Sloat BR, et al. Stearoyl gemcitabine nanoparticles overcome resistance related to the over-expression of ribonucleotide reductase subunit M1. J Control Release. 2012;157:132-40

138. Stella B, Arpicco S, Rocco F, et al. Encapsulation of gemcitabine lipophilic derivatives into polycyanoacrylate nanospheres and nanocapsules. Int J Pharm. 2007;344:71-77

139. Couvreur P, Stella B, Reddy LH, et al. Squalenoyl Nanomedicines as Potential Therapeutics. Nano Lett. 2006;6:2544-2548

140. Reddy LH, Dubernet C, Mouelhi SL, et al. A new nanomedicine of gemcitabine displays enhanced anticancer activity in sensitive and resistant leukemia types. J Control Release. 2007; $124: 20-27$

141. Reddy LH, Marque P-E, Dubernet C, et al. Preclinical Toxicology (Subacute and Acute) and Efficacy of a New Squalenoyl Gemcitabine Anticancer Nanomedicine. J Pharmacol Exp Ther. 2008;325:484-490

142. Kotelevets L, Chastre E, Caron J, et al. A Squalene-Based Nanomedicine for Oral Treatment of Colon Cancer. Cancer Res. 2017;77:2964-2975

143. Maksimenko A, Dosio F, Mougin J, et al. A unique squalenoylated and nonpegylated doxorubicin nanomedicine with systemic long-circulating properties and anticancer activity. Proc Natl Acad Sci USA. 2014;111:E217-26

144. Maksimenko A, Caron J, Mougin J, et al. Gemcitabine-based therapy for pancreatic cancer using the squalenoyl nucleoside monophosphate nanoassemblies. Int J Pharm. 2015;482:38-46

145. Gaudin A, Yemisci M, Eroglu H, et al. Squalenoyl adenosine nanoparticles provide neuroprotection after stroke and spinal cord injury. Nat Nano. 2014;9:1054-1062

146. Abed N, Said-Hassane F, Zouhiri F, et al. An efficient system for intracellular delivery of betalactam antibiotics to overcome bacterial resistance. Sci Rep. 2015;5:13500

147. Semiramoth N, Di Meo C, Zouhiri F, et al. Self-assembled squalenoylated penicillin bioconjugates: an original approach for the treatment of intracellular infections. ACS Nano. 2012;6:3820-31

148. Hillaireau H, Dereuddre-Bosquet N, Skanji R, et al. Anti-HIV efficacy and biodistribution of nucleoside reverse transcriptase inhibitors delivered as squalenoylated prodrug nanoassemblies. Biomaterials. 2013;34:4831-4838

149. Caron J, Reddy LH, Lepetre-Mouelhi S, et al. Squalenoyl nucleoside monophosphate nanoassemblies: new prodrug strategy for the delivery of nucleotide analogues. Bioorg Med Chem Lett. 2010;20:2761-4

150. Othman M, Desmaële D, Couvreur P, et al. Synthesis and physicochemical characterization of new squalenoyl amphiphilic gadolinium complexes as nanoparticle contrast agents. Org Biomol Chem. 2011;9:4367-4386

151. Bui DT, Nicolas J, Maksimenko A, et al. Multifunctional squalene-based prodrug nanoparticles for targeted cancer therapy. Chemical communications (Cambridge, England). 2014;50:53365338

152. Valetti S, Mura S, Noiray M, et al. Peptide Conjugation: Before or After Nanoparticle Formation? Bioconjugate Chem. 2014;25:1971-1983 
153. Valetti S, Maione F, Mura S, et al. Peptide-functionalized nanoparticles for selective targeting of pancreatic tumor. J Control Release. 2014;192:29-39

154. Gopalakrishnan G, Lepetre S, Maksimenko A, et al. Lipid-Conjugation of Endogenous Neuropeptides: Improved Biotherapy against Human Pancreatic Cancer. Adv Healthc Mater. 2015;4:1015-22

155. Raouane M, Desmaele D, Gilbert-Sirieix M, et al. Synthesis, Characterization, and in Vivo Delivery of siRNA-Squalene Nanoparticles Targeting Fusion Oncogene in Papillary Thyroid Carcinoma. J Med Chem. 2011;54:4067-4076

156. Massaad-Massade L, Boutary S, Caillaud M, et al. New Formulation for the Delivery of Oligonucleotides Using "Clickable" siRNA-Polyisoprenoid-Conjugated Nanoparticles: Application to Cancers Harboring Fusion Oncogenes. Bioconjugate Chem. 2018;29:1961-1972

157. Bekkara-Aounallah F, Gref R, Othman M, et al. Novel PEGylated Nanoassemblies Made of Self-Assembled Squalenoyl Nucleoside Analogues. Adv Funct Mater. 2008;18:3715-3725

158. Rautio J, Meanwell NA, Di L, et al. The expanding role of prodrugs in contemporary drug design and development. Nat Rev Drug Discov. 2018;17:559

159. Mura S, Bui DT, Couvreur P, et al. Lipid prodrug nanocarriers in cancer therapy. J Control Release. 2015;208:25-41

160. Epaule C, Maksimenko A, Bastian G, et al. X-ray microfluorescence for biodistribution studies of nanomedicines. Int J Pharm. 2017;531:343-349

161. Skarbek C, Lesueur LL, Chapuis H, et al. Preactivated Oxazaphosphorines Designed for Isophosphoramide Mustard Delivery as Bulk Form or Nanoassemblies: Synthesis and Proof of Concept. J Med Chem. 2015;58:705-717

162. Reddy LH, Khoury H, Paci A, et al. Squalenoylation Favorably Modifies the in Vivo Pharmacokinetics and Biodistribution of Gemcitabine in Mice. Drug Metab Dispos. 2008;36:1570-1577

163. Reddy LH, Renoir J-M, Marsaud V, et al. Anticancer Efficacy of Squalenoyl Gemcitabine Nanomedicine on 60 Human Tumor Cell Panel and on Experimental Tumor. Mol Pharm. 2009;6:1526-1535

164. Buchy E, Vukosavljevic B, Windbergs M, et al. Synthesis of a deuterated probe for the confocal Raman microscopy imaging of squalenoyl nanomedicines. Beilstein $J$ Org Chem. 2016;12:1127-1135

165. Caron J, Maksimenko A, Wack S, et al. Improving the antitumor activity of squalenoylpaclitaxel conjugate nanoassemblies by manipulating the linker between paclitaxel and squalene. Adv Healthc Mater. 2013;2:172-85

166. Caron J, Maksimenko A, Mougin J, et al. Combined antitumoral therapy with nanoassemblies of bolaform polyisoprenoyl paclitaxel/gemcitabine prodrugs. Polym Chem. 2014;5:1662-1673

167. Mura S, Buchy E, Askin G, et al. In vitro investigation of multidrug nanoparticles for combined therapy with gemcitabine and a tyrosine kinase inhibitor: Together is not better. Biochimie. 2016;130:4-13

168. Gaudin A, Tagit O, Sobot D, et al. Transport Mechanisms of Squalenoyl-Adenosine Nanoparticles Across the Blood-Brain Barrier. Chem Mater. 2015;27:3636-3647

169. Cheikh-Ali Z, Caron J, Cojean S, et al. "Squalenoylcurcumin" Nanoassemblies as WaterDispersible Drug Candidates with Antileishmanial Activity. ChemMedChem. 2015;10:411-418

170. Maksimenko A, Mougin J, Mura S, et al. Polyisoprenoyl gemcitabine conjugates self assemble as nanoparticles, useful for cancer therapy. Cancer Lett. 2013;334:346-53

171. Mura S, Zouhiri F, Lerondel S, et al. Novel isoprenoyl nanoassembled prodrug for paclitaxel delivery. Bioconjugate Chem. 2013;24:1840-9

172. Van Tamelen EE. Bioorganic chemistry: sterols and acrylic terpene terminal expoxides. Accounts of Chemical Research. 1968;1:111-120

173. Desmaële D, Gref R, Couvreur P. Squalenoylation: A generic platform for nanoparticular drug delivery. J Control Release. 2012;161:609-618

174. Kingston DG. Recent advances in the chemistry of taxol. J Nat Prod. 2000;63:726-34

175. Zhu Q, Guo Z, Huang N, et al. Comparative molecular field analysis of a series of paclitaxel analogues. J Med Chem. 1997;40:4319-28 
176. Dosio F, Reddy LH, Ferrero A, et al. Novel Nanoassemblies Composed of Squalenoyl-Paclitaxel Derivatives: Synthesis, Characterization, and Biological Evaluation. Bioconjugate Chem. 2010;21:1349-1361

177. Arias JL, Reddy LH, Othman M, et al. Squalene Based Nanocomposites: A New Platform for the Design of Multifunctional Pharmaceutical Theragnostics. ACS Nano. 2011;5:1513-1521

178. Stork G, Burgstahler AW. The Stereochemistry of Polyene Cyclization. J Am Chem Soc. 1955;77:5068-5077

179. Couvreur P, Reddy LH, Mangenot S, et al. Discovery of New Hexagonal Supramolecular Nanostructures Formed by Squalenoylation of an Anticancer Nucleoside Analogue. Small. 2008;4:247-253

180. Mackey JR, Mani RS, Selner M, et al. Functional nucleoside transporters are required for gemcitabine influx and manifestation of toxicity in cancer cell lines. Cancer Res. 1998;58:434957

181. Kufe DW, Major PP, Egan EM, et al. Correlation of cytotoxicity with incorporation of ara-C into DNA. J Biol Chem. 1980;255:8997-8900

182. Iwasaki H, Huang P, Keating MJ, et al. Differential Incorporation of Ara-C, Gemcitabine, and Fludarabine Into Replicating and Repairing DNA in Proliferating Human Leukemia Cells. Blood. 1997;90:270-278

183. Sobot D, Mura S, Rouquette M, et al. Circulating Lipoproteins: A Trojan Horse Guiding Squalenoylated Drugs to LDL-Accumulating Cancer Cells. Mol Ther. 2017;25:1596-1605

184. Sobot D, Mura S, Yesylevskyy SO, et al. Conjugation of squalene to gemcitabine as unique approach exploiting endogenous lipoproteins for drug delivery. Nat Commun. 2017;8:15678

185. Sobot D, Mura S, Couvreur P. Nanoparticles: Blood Components Interactions. In: Kobayashi S, Müllen K, editors. Encyclopedia of Polymeric Nanomaterials. Berlin, Heidelberg: Springer Berlin Heidelberg; 2014. p. 1-10.

186. Nel AE, Madler L, Velegol D, et al. Understanding biophysicochemical interactions at the nanobio interface. Nat Mater. 2009;8:543-557

187. Lundqvist M, Stigler J, Elia G, et al. Nanoparticle size and surface properties determine the protein corona with possible implications for biological impacts. Proc Natl Acad Sci USA. 2008; $105: 14265-14270$

188. Kapralov AA, Feng WH, Amoscato AA, et al. Adsorption of Surfactant Lipids by SingleWalled Carbon Nanotubes in Mouse Lung upon Pharyngeal Aspiration. ACS Nano. 2012;6:4147-4156

189. Hellstrand E, Lynch I, Andersson A, et al. Complete high-density lipoproteins in nanoparticle corona. FEBS Journal. 2009;276:3372-3381

190. Brown MS, Goldstein JL. A receptor-mediated pathway for cholesterol homeostasis. Science. 1986;232:34-47

191. Vitols S, Gahrton G, Ost A, et al. Elevated low density lipoprotein receptor activity in leukemic cells with monocytic differentiation. Blood. 1984;63:1186-1193

192. Vitols S, Söderberg-Reid K, Masquelier M, et al. Low density lipoprotein for delivery of a water-insoluble alkylating agent to malignant cells. In vitro and in vivo studies of a druglipoprotein complex. Br J Cancer. 1990;62:724-729

193. Versluis AJ, van Geel PJ, Oppelaar H, et al. Receptor-mediated uptake of low-density lipoprotein by B16 melanoma cells in vitro and in vivo in mice. Br J Cancer. 1996;74:525-532

194. Gal D, Macdonald PC, Porter JC, et al. Cholesterol metabolism in cancer cells in monolayer culture. III. Low-density lipoprotein metabolism. Int J Cancer. 1981;28:315-319

195. Ho YK, Brown S, Bilheimer DW, et al. Regulation of low density lipoprotein receptor activity in freshly isolated human lymphocytes. J Clin Invest. 1976;58:1465-74

196. Firestone RA. Low-density lipoprotein as a vehicle for targeting antitumor compounds to cancer cells. Bioconjugate Chem. 1994;5:105-13

197. Oschry Y, Eisenberg S. Rat plasma lipoproteins: re-evaluation of a lipoprotein system in an animal devoid of cholesteryl ester transfer activity. J Lipid Res. 1982;23:1099-106

198. Cayre F, Mura S, Andreiuk B, et al. In Vivo FRET Imaging to Predict the Risk Associated with Hepatic Accumulation of Squalene-Based Prodrug Nanoparticles. Adv Healthc Mater. 2018;7:1700830 
199. Samadi-Baboli M, Favre G, Bernadou J, et al. Comparative study of the incorporation of ellipticine-esters into low density lipoprotein (LDL) and selective cell uptake of drug--LDL complex via the LDL receptor pathway in vitro. Biochem Pharmacol. 1990;40:203-12

200. Ng KK, Lovell JF, Zheng G. Lipoprotein-inspired nanoparticles for cancer theranostics. Acc Chem Res. 2011;44:1105-13

201. Kader A, Pater A. Loading anticancer drugs into HDL as well as LDL has little affect on properties of complexes and enhances cytotoxicity to human carcinoma cells. J Control Release. 2002;80:29-44

202. Rensen PCN, de Vrueh RLA, Kuiper J, et al. Recombinant lipoproteins: lipoprotein-like lipid particles for drug targeting. Adv Drug Deliv Rev. 2001;47:251-276

203. Christian DA, Cai S, Garbuzenko OB, et al. Flexible Filaments for in Vivo Imaging and Delivery: Persistent Circulation of Filomicelles Opens the Dosage Window for Sustained Tumor Shrinkage. Mol Pharm. 2009;6:1343-1352

204. Boison D. Adenosine as a neuromodulator in neurological diseases. Curr Opin Pharmacol. 2008;8:2-7

205. Williams-Karnesky RL, Stenzel-Poore MP. Adenosine and Stroke: Maximizing the Therapeutic Potential of Adenosine as a Prophylactic and Acute Neuroprotectant. Curr Neuropharmacol. 2009;7:217-227

206. de Mendonça A, Sebastião AM, Ribeiro JA. Adenosine: does it have a neuroprotective role after all? Brain Res Rev. 2000;33:258-274

207. Fredholm BB, Chen J-F, Cunha RA, et al. Adenosine and Brain Function. International Review of Neurobiology. Vol. 63: Academic Press; 2005. p. 191-270.

208. Moser GH, Schrader J, Deussen A. Turnover of adenosine in plasma of human and dog blood. Am J Physiol. 1989;256:C799-806

209. Gomes CV, Kaster MP, Tome AR, et al. Adenosine receptors and brain diseases: neuroprotection and neurodegeneration. Biochim Biophys Acta. 2011;1808:1380-99

210. Isakovic AJ, Abbott NJ, Redzic ZB. Brain to blood efflux transport of adenosine: blood-brain barrier studies in the rat. J Neurochem. 2004;90:272-86

211. Bildstein L, Dubernet C, Marsaud V, et al. Transmembrane diffusion of gemcitabine by a nanoparticulate squalenoyl prodrug: An original drug delivery pathway. J Control Release. 2010;147:163-170

212. Kwon BK, Hillyer J, Tetzlaff W. Translational Research in Spinal Cord Injury: A Survey of Opinion from the SCI Community. J Neurotrauma. 2010;27:21-33

213. Hanahan D. Heritable formation of pancreatic $\beta$-cell tumours in transgenic mice expressing recombinant insulin/simian virus 40 oncogenes. Nature. 1985;315:115 